\title{
Efficient DC Analysis Using Model Order Reduction
}

\author{
by \\ Qi Sun, B.Sc., M.Sc. \\ A thesis submitted to the Faculty of Graduate Studies and Postdoctoral Affairs \\ in partial fulfillment of the requirements for the degree of \\ Master of Applied Science \\ in \\ Electrical and Computer Engineering \\ Ottawa-Carleton Institute for Electrical and Computer Engineering \\ Department of Electronics \\ Faculty of Engineering \\ Carleton University \\ Ottawa, Ontario, Canada \\ January 2017
}

Copyright (c) 2017

Qi Sun 


\section{Abstract}

For nonlinear circuits, DC analysis is one of the circuit simulation applications. The conventional source stepping continuation method is computationally expensive to do the Newton-Raphson iteration due to the large numbers of nonlinear functions and the inversion of a large matrix. It is also hard to find an efficient algorithm to do the Model Order Reduction (MOR), DC analysis and the parameterized analysis for nonlinear systems.

To address the above difficulties, a novel method is proposed in this thesis for the DC analysis and the parameterized analysis. This new approach reduces the computational cost by applying the model order reduction technique to the original model. The model size of the reduced order model is much smaller, thus the computational time will be saved during the Newton-Raphson iteration. In addition, the proposed method solves the MOR problem for the nonlinear vector by applying the DEIM algorithm. Furthermore, the format of the DC solution parameterized analysis is provided. To the end, basing on the new circuit equation format, a method to compute the moment subspace is presented after applying the DEIM approximation. Pertinent numerical results are presented to verify the proposed algorithm. The simulation result also demonstrates the efficiency of the new method to solve the nonlinear equations. 


\section{Acknowledgments}

My deepest appreciation goes first and foremost to Professor Michel Nakhla, who is my supervisor. His personality was one of the reasons that led me to choose CAD as my research area. With his abundant knowledge and foresight for the computer aided design area, he guided me through this fascinating research topic, which was an absolute enjoyable adventure. There were several times that I met difficulty in my research, but with his continuing encouragement, unconditional support and valuable advice, I was able to succeed.

I would also like to express my heartfelt appreciation to my co-supervisor, Dr. Behzad Nouri. With his consistent and enlightening instruction, I developed a deeper and better understanding of my research. The thesis and research could not have reached this integrated level without his guidance.

I would also like to express my gratitude to Professor Emad Gad for clarifying the thesis topic. His selfless assistance of the program installation and the demo function elevated my research. His helpful illustration improved the quality of the work overall.

Additionally, thanks also to the CAD team, Kai Guo and Ye Tao. I will not forget the many interesting and inspiring discussions we had, not only about my research, but about my post-graduate life at Carleton. I treasure our friendship.

I would like to express my deep love for my parents and relatives, for their unconditional love and support. Their spiritual and financial support were the foundation 
to my success. Although we are miles apart, they are here with me, and I dedicate this work to them. 


\section{Table of Contents}

Abstract $\quad$ ii

Acknowledgments

Table of Contents $\quad$ v

List of Tables $\quad$ viii

List of Figures $\quad$ ix

List of Acronyms $\quad$ xi

List of Symbols $\quad$ xiii

1 Introduction 1

1.1 Background and Motivation . . . . . . . . . . . . 1

1.2 Objectives and Contributions $\ldots \ldots \ldots \ldots \ldots$

1.3 Organization of the Thesis $\ldots \ldots \ldots \ldots \ldots \ldots$

2 DC Analysis of Nonlinear Circuits 5

2.1 Formulation of Nonlinear Circuit Equations $\ldots \ldots \ldots \ldots$

2.2 Newton-Raphson Iteration to Solve Nonlinear Equations $\ldots \ldots$

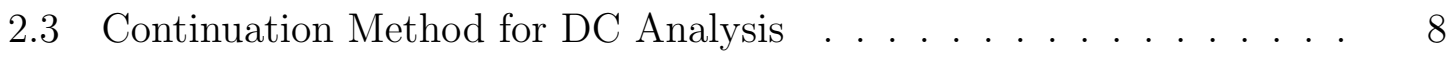


3 Model Order Reduction Techniques

3.1 Introduction . . . . . . . . . . . . . . . . . 11

3.2 Model Order Reduction for Linear Circuits . . . . . . . . . . . . . . . 12

3.3 Krylov-Subspace based Model Order Reduction . . . . . . . . . . . . 14

3.4 POD-Galerkin Model Order Reduction . . . . . . . . . . . . . 15

3.4.1 Introduction of the POD Basis . . . . . . . . . . . 15

3.5 Model Order Reduction for Nonlinear Circuits . . . . . . . . . . . . . 16

3.5.1 Projection Framework for Nonlinear MOR . . . . . . . . . . 16

3.5.2 Challenges of Projection Framework . . . . . . . . . . . . 18

3.5.3 POD-Galerkin Complexity Problem . . . . . . . . . . . . . 19

4 Nonlinear Model Order Reduction for DC Solution 21

4.1 Computation of the Moments in the Taylor Series Expansions . . . . 22

4.1.1 Evaluation of the Moments for Nonlinear Function . . . . . . 24

4.1.2 Evaluation of the Moments for DC Solution . . . . . . . . . 28

4.1.3 Summary for Moments . . . . . . . . . . . . . . . . . 31

4.2 Reduced Model via Congruent Transformation . . . . . . . . . . . . . 32

5 Review of Nonlinear Model Reduction via Discrete Empirical In$\begin{array}{ll}\text { terpolations (DEIM) } & 34\end{array}$

5.1 Problem Formulation . . . . . . . . . . . . . . . . . . . . 35

5.2 Formulation of DEIM . . . . . . . . . . . . . . . 36

5.2.1 Approximation of the Nonlinear Function . . . . . . . . . . . 36

5.2.2 Approximation of the Jacobian Function . . . . . . . . . . . . 42

5.3 Analysis and Algorithm for Interpolation Indices . . . . . . . . . . . . 43

5.4 Interpolation of General Nonlinear Functions . . . . . . . . . . . . . . 44

5.5 Computational Results and Verification . . . . . . . . . . . . 46 
$5.5 .1 \quad$ Example I . . . . . . . . . . . . . . . . . . . . . 47

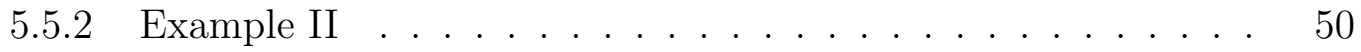

6 Proposed Model Order Reduction for DC Analysis 54

6.1 Computation of the Nonlinear Function Moments . . . . . . . 55

6.1.1 Computation of the Jacobian Function Moments . . . . . . 55

6.1.2 Computation of the Nonlinear Function Moments . . . . . 57

6.2 Nonlinear Model Order Reduction with DEIM . . . . . . . . . . . . 59

6.3 Modification of the Reduced Model . . . . . . . . . . . . . . . 61

6.4 Computational Results and Verification . . . . . . . . . . . 63

6.4 .1 Example I . . . . . . . . . . . . . . . . . . . 63

6.4 .2 Example II . . . . . . . . . . . . . . . . . . 65

7 Parametrized Model Order Reduction $\quad 70$

7.1 Parameterized Analysis for Nonlinear DC Solution . . . . . . . . . 71

7.2 Reduced Model Formation for DC parameterized Analysis . . . . . 72

7.3 Computation of Derivative and Moments . . . . . . . . . 73

7.4 Computation Results and Verification . . . . . . . . . . 77

7.4 .1 Example I . . . . . . . . . . . . . . . . . . . . 77

7.4 .2 Example II . . . . . . . . . . . . . . . . . . . . . 81

$\begin{array}{lll}8 & \text { Conclusion and Future Work } & 87\end{array}$

8.1 Conclusion . . . . . . . . . . . . . . . . 87

8.2 Future Work . . . . . . . . . . . . . . . . . . . . . . . . . . . . 89

$\begin{array}{lr}\text { List of References } & 90\end{array}$ 


\section{List of Tables}

4.1 Summary . . . . . . . . . . . . . . . . . . . 31

5.1 POD Method for Example I . . . . . . . . . . . . . . . . 48

5.2 POD-DEIM Method for Example I . . . . . . . . . . . . . . . 49

5.3 POD Method for Example II . . . . . . . . . . . . . . . . . . 51

5.4 POD-DEIM Method for Example II . . . . . . . . . . . . . . 52

6.1 Moment Subspace Method for Example I . . . . . . . . . . . . . . . 64

6.2 Moment Subspace DEIM Method for Example I . . . . . . . . . . . 65

6.3 Moment Subspace Method for Example II . . . . . . . . . . . 66

6.4 Moment Subspace DEIM Method for Example II . . . . . . . . . 67

6.5 Modified Moment Subspace DEIM Method for Example II . . . . . 68

7.1 Example I: Positive Parameterized Analysis _ . . . . . . . . . 78

7.2 Example I: Negative Parameterized Analysis . . . . . . . . . . . 80

7.3 Example II: Positive Parameterized Analysis . . . . . . . . . . . . . 82

7.4 Example II: Negative Parameterized Analysis _ . . . . . . . . . . 84 


\section{List of Figures}

2.1 (a) Tuned BJT Amplifier Circuit (Example I), (b) Crystal Equivalent

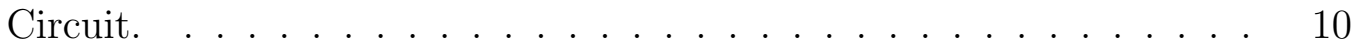

5.1 Ebers-Moll Model . . . . . . . . . . . . . . . . . . . . . . . . . 47

5.2 Example I: POD DC Analysis . . . . . . . . . . . . . . . . 48

5.3 Example I: POD-DEIM DC Analysis . . . . . . . . . . . . . . . 49

5.4 Diode Ladder Circuit (Example II) . . . . . . . . . . . . . . . 50

5.5 Example II: POD DC Analysis _ . . . . . . . . . . . . . . . . 51

5.6 Example II: POD-DEIM DC Analysis . . . . . . . . . . . . . . . 52

6.1 Example I: Moment Subspace for DC Analysis . . . . . . . . . . . 63

6.2 Example I: Moment Subspace DEIM for DC Analysis . . . . . . . . . 64

6.3 Example II: Moment Subspace with 20 Moments for DC Analysis . . 66

6.4 Example II: Moment Subspace DEIM with 25 Moments 25 Selections for DC Analysis . . . . . . . . . . . . . . . 67

6.5 Example II: Modified Moment Subspace DEIM with 5 Moments 5 Selections . . . . . . . . . . . . . . . . . . . . 68

7.1 Example I: Positive Parameterized Analysis Result _ . . . . . . 78

7.2 Example I: Positive Parameterized Analysis Result _ . . . . . . . 79

7.3 Example I: Negative Parameterized Analysis Result . . . . . . . . . 80

7.4 Example I: Negative Parameterized Analysis Result . . . . . . . . . . 81 
7.5 Example II: Positive Parameterized Analysis . . . . . . . . . . . . . 82

7.6 Example II: Positive Parameterized Analysis Result . . . . . . . . . . 83

7.7 Example II: Negative Parameterized Analysis with 5 Moments-10 Se-

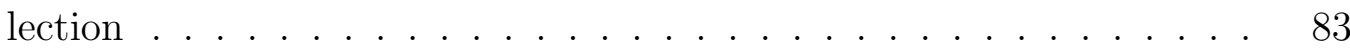

7.8 Example II: Negative Parameterized Analysis Result with 5 Moments10 Selection . . . . . . . . . . . . . . . . . . 84

7.9 Example II: Negative Parameterized Analysis with 7 Moments-15 Se-

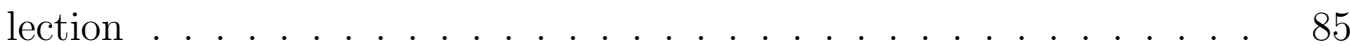

7.10 Example II: Negative Parameterized Analysis Result with 7 Moments15 Selection . . . . . . . . . . . . . . . 86 


\section{List of Acronyms}

\begin{tabular}{|c|c|}
\hline Acronyms & Definition \\
\hline AWE & Asymptotic Waveform Evaluation \\
\hline CAD & Computer Aided Design \\
\hline $\mathrm{DC}$ & Direct Current \\
\hline DEIM & Discrete Empirical Interpolation Method \\
\hline EM & Electro-Magnetic \\
\hline FD & Finite Difference \\
\hline $\mathrm{IC}$ & Integrated Circuit \\
\hline KCL & Kirchoff's Current Law \\
\hline $\mathrm{LU}$ & Lower Upper \\
\hline MNA & Modified Nodal Analysis \\
\hline MMT & Moment Matching Technique \\
\hline MOR & Model Order Reduction \\
\hline ODE & Ordinary Differential Equation \\
\hline PMOR & Parametrized Model-Order Reduction \\
\hline
\end{tabular}


POD Proper Orthogonal Decomposition Method

ROM Reduced-Order Model

SVD Singular Value Decomposition

VLSI Very Large-Scale Integration 


\section{List of Symbols}

\begin{tabular}{|c|c|}
\hline Symbols & Definition \\
\hline$\sigma$ & standard deviation \\
\hline G & matrix describing the memoryless elements in the circuit \\
\hline $\mathrm{C}$ & matrix describing the memory elements in the circuit \\
\hline $\mathbf{x}$ & vector of variables of nonlinear circuit \\
\hline$\hat{\mathbf{x}}$ & vector of reduced model variables of nonlinear circuit \\
\hline$\tilde{\mathbf{x}}$ & vector of approximate variables of nonlinear circuit \\
\hline $\mathbf{L}$ & selector matrix for the output port of the circuit \\
\hline b & vector describing the input elements in the circuit \\
\hline$h$ & step size of the simulation \\
\hline$\lambda$ & $\begin{array}{l}\text { diagonal matrix whose diagonal elements are eigenvalues } \\
\text { of nonlinear moments }\end{array}$ \\
\hline $\mathbf{I}$ & identity matrix \\
\hline$K$ & Krylov subspace \\
\hline colspan & columns expansion \\
\hline
\end{tabular}




\begin{tabular}{ll}
$\mathbf{Q}$ & orthogonal basis \\
$\alpha$ & parameter for circuit analysis \\
$\mathbf{M}$ & moments of the variable \\
$\widehat{\mathbf{G}}$ & reduced order model of $\mathrm{G}$ \\
$\widehat{\mathbf{C}}$ & reduced order model of $\mathrm{C}$ \\
$\widehat{\mathbf{b}}$ & reduced order model of $\mathrm{b}$ \\
$\mathbf{f}(\mathbf{x})$ & nonlinear function \\
$\mathbf{J}(\mathbf{x})$ & Jacobian function \\
$\boldsymbol{\zeta}$ & Jacobian function of the circuit parameter \\
$\mathbf{F}_{\mathbf{k}}$ & Kth moment of the nonlinear function \\
$\mathbf{J}_{\mathbf{k}}$ & Kth moment of the Jacobian function \\
$\mathbf{U}$ & projection base of the nonlinear function \\
$\mathbf{\Gamma}$ & nonlinear function interpolation indices \\
$\mathbf{P}$ & DEIM selection matrix \\
\hline
\end{tabular}




\section{Chapter 1}

\section{Introduction}

\subsection{Background and Motivation}

Currently, the background of the computer hardware, mobile devices, and control system are closely bonded with manufacturing and people's daily life. According to Moore's law, the number of the transistors required in the integrated circuit doubles about every two years, and the pattern thought to be a relative approximation for decades. The computer aided design (CAD) techniques have been developing in a dramatic speed for very large scale integration (VLSI) circuits. The electronic system uses a device dimension that in nano-scale region and the signal are in the gigahertz range. The continuous research for the CAD area is necessary for the complex system models and the high performance of the circuit.

Designers of the hardware, system, and electronic circuits need simulation tools to check and refine their design in a convenient and economic way. The efficient

algorithm for the circuit simulation could be used for the circuit simulation software or the system design tools. Thus more advanced theory could have a significant positive effect on the design of the system. There are many techniques developed for linear systems; however, the CAD area still needs the development of simulation 
algorithms for nonlinear systems.

Model Order Reduction (MOR) techniques have been proved my many applications for its great efficiency of simulating large scale systems. The reduction is done by projecting the original large number of equations to space which has much less dimension using the orthogonal congruence transformation. As for the current techniques to find the moment subspace of the variable for nonlinear circuits, the Proper Orthogonal Decomposition (POD) with Galerkin projection is widely used [1]; however, it needs the circuit testing and that is why it might not be a proper method for the DC analysis. The most widely used projection technique is based on the moment matching, which offers a way to find the moments of variable with respect to frequency. Thus the projection matrix could be built using orthogonal bases of the moment subspace spanned by moment vectors [2]. This method to find the moment could be transplanted for the nonlinear circuit MOR.

Additionally, some techniques have also been published for the evaluation and approximation of the nonlinear function. The Discrete Empirical Interpolation Method (DEIM) is introduced in the thesis [3]. It is the further development basing on the Empirical Interpolation Method (EIM) [4]. The DEIM approach is to create the approximated nonlinear function which can be established by some entries of the original nonlinear function vector. The technique uses the moment subspace of the nonlinear function for the projection and the selection. The proposed method overcomes the difficulties that the evaluation has to be built in the original domain and the expensive MOR process for the nonlinear functions and the Jacobian matrix. By applying the DEIM, the ROM could be found from the moment subspace easily. 


\subsection{Objectives and Contributions}

The thesis presents a novel algorithm for the Model Order Reduction of nonlinear circuits. An efficient method has been developed for the DC analysis and the parameterized analysis. Main contributions and advantages can be explained by developing the following points:

- Modeling the DC analysis formula with the source vector multiplier, and variables Taylor Series expansion is expanded in respect of the parameter. Moments with a high order are found and the moment subspace is formed.

- By adapting the Leibniz theorem, moments for nonlinear functions is found with the variable moments.

- Application of Model Order Reduction algorithm is used to both DC analysis and parameterized analysis formula.

- The DEIM algorithm is applied for the nonlinear moments to deduct the cost of nonlinear function evaluation.

- Formula of the parameterized analysis is built, and the new variable moments is found to form the moment subspace.

The contribution of the thesis leads to a method which can perform the DC analysis and the parameterized analysis with ensured accurate results and reduced computational cost. Additionally, the proposed algorithm gives a method to the lost cost nonlinear function vector MOR. 


\subsection{Organization of the Thesis}

Chapter 2 has a brief review for techniques and the circuit mathematical model which is used for the DC analysis. Chapter 3 gives an introduction on the existing Model Order Reduction techniques and the POD-Galerkin approach. Chapter 4 provides the algorithm to find the moments for the nonlinear DC analysis. Chapter 5 provides a review on the DEIM theory. It gives the format to apply the nonlinear function evaluation efficiently. Chapter 6 provides a new method combining the moment subspace method and the DEIM theory. It offers a way to find moments for the nonlinear function. Chapter 7 proposes the method to conduct the parameterized analysis for nonlinear circuits. The conclusion and the future work is presented in Chapter 8 . 


\section{Chapter 2}

\section{Analysis of Nonlinear Circuits}

$\mathrm{DC}$ analysis is a challenging task in circuit simulation. This chapter describes the mathematical formulation of the DC equations based on the Modified-Nodal Analysis (MNA) and various techniques proposed in the literature for solving these equations $[5-9]$.

\subsection{Formulation of Nonlinear Circuit Equations}

The MNA equations for a general nonlinear circuit can be written in the form:

$$
\begin{aligned}
& \mathbf{G} \mathbf{x}(t)+\mathbf{C} \frac{d \mathbf{x}(t)}{d t}+\mathbf{f}(\mathbf{x}(t))=\mathbf{b}(t) \\
& \mathbf{b}(t)=\mathbf{B} \mathbf{u}(t) \\
& \mathbf{y}(t)=\mathbf{L x}(t)
\end{aligned}
$$

where $\mathbf{x}(t) \in \mathbb{R}^{n}$ contains the node voltages and some branch currents of the circuit, $\mathbf{u}(t) \in \mathbb{R}^{p}$ contains the information of stimuli at inputs of the circuit, $\mathbf{y}(t) \in \mathbb{R}^{q}$ is the selected output of the circuit and $\mathbf{L} \in \mathbb{R}^{q \times n}$ and $\mathbf{B} \in \mathbb{R}^{n \times p}$ are selector matrices. $\mathbf{G}, \mathbf{C} \in \mathbb{R}^{n \times n}$ are conductance and susceptance matrices, respectively. 
For the DC analysis, the circuit equation can be written as:

$$
\begin{aligned}
& \mathbf{G} \mathbf{x}+\mathbf{f}(\mathbf{x})=\mathbf{b} \\
& \mathbf{b}=\mathbf{B u} \\
& \mathbf{y}=\mathbf{L x}
\end{aligned}
$$

Newton-Raphson (N-R) iterative algorithm is the most common method used in the literature and commercial simulators for solving (2.2). However, Newton's iterations suffer from several drawbacks as illustrated in the next section.

\subsection{Newton-Raphson Iteration to Solve Nonlinear Equations}

Newton-Raphson (N-R) iterations for solving (2.2) can be written in the form:

$$
\left.\boldsymbol{\Psi}(\mathbf{x})\right|_{\mathbf{x}=\mathbf{x}_{\mathbf{i}}} \Delta \mathbf{x}_{i}=-\left.\boldsymbol{\Phi}(\mathbf{x})\right|_{\mathbf{x}=\mathbf{x}_{\mathbf{i}}} \quad \text { for } \quad i=0,1, \cdots
$$

where

$$
\begin{aligned}
& \mathbf{\Phi}(\mathbf{x})=\mathbf{G} \mathbf{x}+\mathbf{f}(\mathbf{x})-\mathbf{b} \\
& \boldsymbol{\Psi}(\mathbf{x})=\mathbf{G}+\mathbf{J}(\mathbf{x})
\end{aligned}
$$

where $\mathbf{J}(\mathbf{x}) \in \mathbb{R}^{n \times n}$ is the Jacobian matrix:

$$
\mathbf{J}(\mathbf{x})=\frac{d \mathbf{f}(\mathbf{x})}{d \mathbf{x}}
$$

The subscript $i$ represents the $i$ th iteration where $\mathbf{x}_{\mathbf{0}}$ is the initial guess.

Convergence is achieved under the following two conditions: 


$$
\begin{aligned}
& \text { i) } \quad\left\|\mathbf{x}_{i+1}-\mathbf{x}_{i}\right\|<\varepsilon_{1} \\
& \text { ii) } \quad\left\|\boldsymbol{\Phi}\left(\mathbf{x}_{i+1}\right)\right\|<\varepsilon_{2}
\end{aligned}
$$

where $\varepsilon_{1}$ and $\varepsilon_{2}$ are pre-specified error tolerances, and the absolute calculation is a vector norm on $\mathbb{R}^{n}$.

The convergence of (2.3) is highly dependent on the selection of the initial guess $\mathbf{x}_{\mathbf{0}}$. In absence of good initial guess, N-R method requires a significant number of computationally costly iterations to converge or it completely fails. Note that solving (2.3) at each iteration requires LU decomposition of the matrix $\boldsymbol{\Psi}$ and forward/backward substitution.

The computational steps of the Newton-Raphson Algorithm for solving (2.2) are summarized in Algorithm 1, presented using MATLAB-like pseudocode.

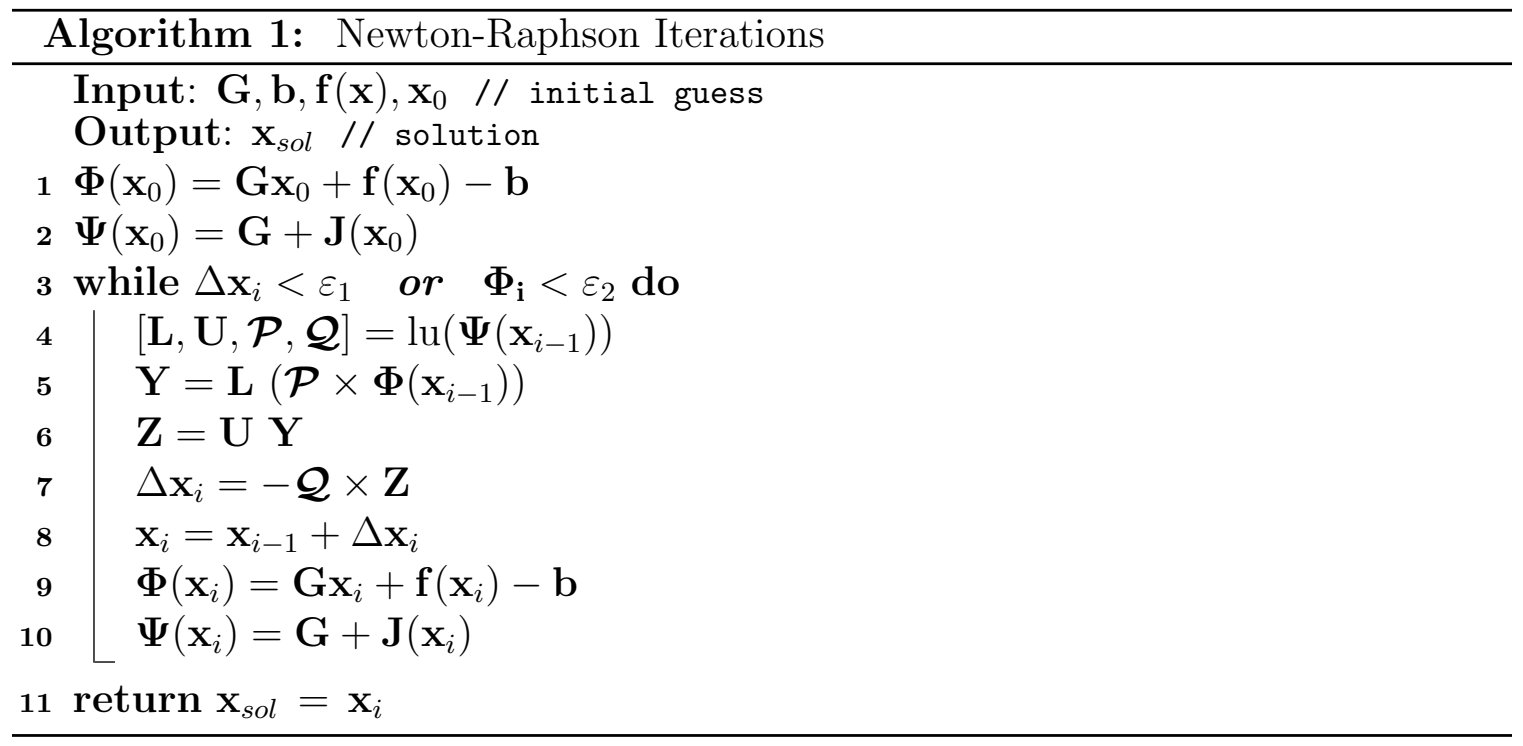

As an iterative method, Newton-Raphson iteration generates a sequence $\left\{x^{(k)}\right\}_{k=0}^{\infty}$ of candidate solutions, and it stops when the sequence converges to a solution $x^{*}$. It is defined that the sequence converges q-linearly to $x^{*}$ if there exists a number $c \in(0,1)$ 
such that:

$$
\lim _{k \rightarrow \infty} \frac{\left|x^{k+1}-x^{*}\right|}{\left|x^{k}-x^{*}\right|}=c
$$

The $\mathrm{c}$ is called the asymptotic error constant. The ' $\mathrm{q}$ ' stands for the word 'quotient' because the ratio used to define the convergence in (2.6). If the sequence converges q-linearly, the rate of the converge can be distinguished as convergence order.

$$
\lim _{k \rightarrow \infty} \frac{\left|x^{k+1}-x^{*}\right|}{\left|x^{k}-x^{*}\right|^{\alpha}}=c
$$

where the $\alpha$ is the convergence order. The convergence with order 2 is called as qquadratic convergence. With a q-quadratic method, the number of correct significant digits is approximately doubled with every iteration. It is shown in the book [10] that the Newton-Raphson method converges q-quadratically to $x^{*}$. However, if $f^{\prime}\left(x^{*}\right)=0$, Newton-Raphson method still converges but only q-linearly which has a convergence order 1.

\subsection{Continuation Method for DC Analysis}

One of the popular methods for increasing the probability of convergence is based on source ramping [9], which is a special case of general techniques known in the literature as continuation methods. In its simplest form, using the source ramping strategy starts with a low value for the DC source and (2.3) is solved assuming $\mathbf{x}_{\mathbf{0}}=0$. The value of the source is ramped-up in stages, where the solution at a given stage is used as an initial guess for the next stage.

Mathematically, source ramping is equivalent to solving a modified form of (2.3) 
as

$$
\Phi(\mathbf{x}, \alpha)=\mathbf{G} \mathbf{x}+\mathbf{f}(\mathbf{x})-\alpha \mathbf{b}=0
$$

where $\alpha$ is the multiplier of the source vector, and it increases gradually from $\alpha=0$ to $\alpha=1$.

Although source ramping significantly increases the probability of convergence, it is computationally very expensive since it requires solutions of different DC equations corresponding to the different values of the DC source.

As an illustrative example, it is to be noted that, the direct application of N-R iteration to a tuned-amplifier circuit in Fig. 2.1 fails to converge. However using the source ramping technique, convergence is achieved in 10 steps (for $\alpha=0.1, \ldots, 1$ ). The total number of required N-R iterations throughout the steps is 74 while each $\mathrm{N}-\mathrm{R}$ iteration requires the solution of algebraic matrix equations of size $19 \times 19$.

In [11], a method is proposed based on the continuation technique and modelorder reduction. The objective of this method is to scale-down the cost of iterations using MOR techniques. A brief background about MOR is presented in the next Chapter. 


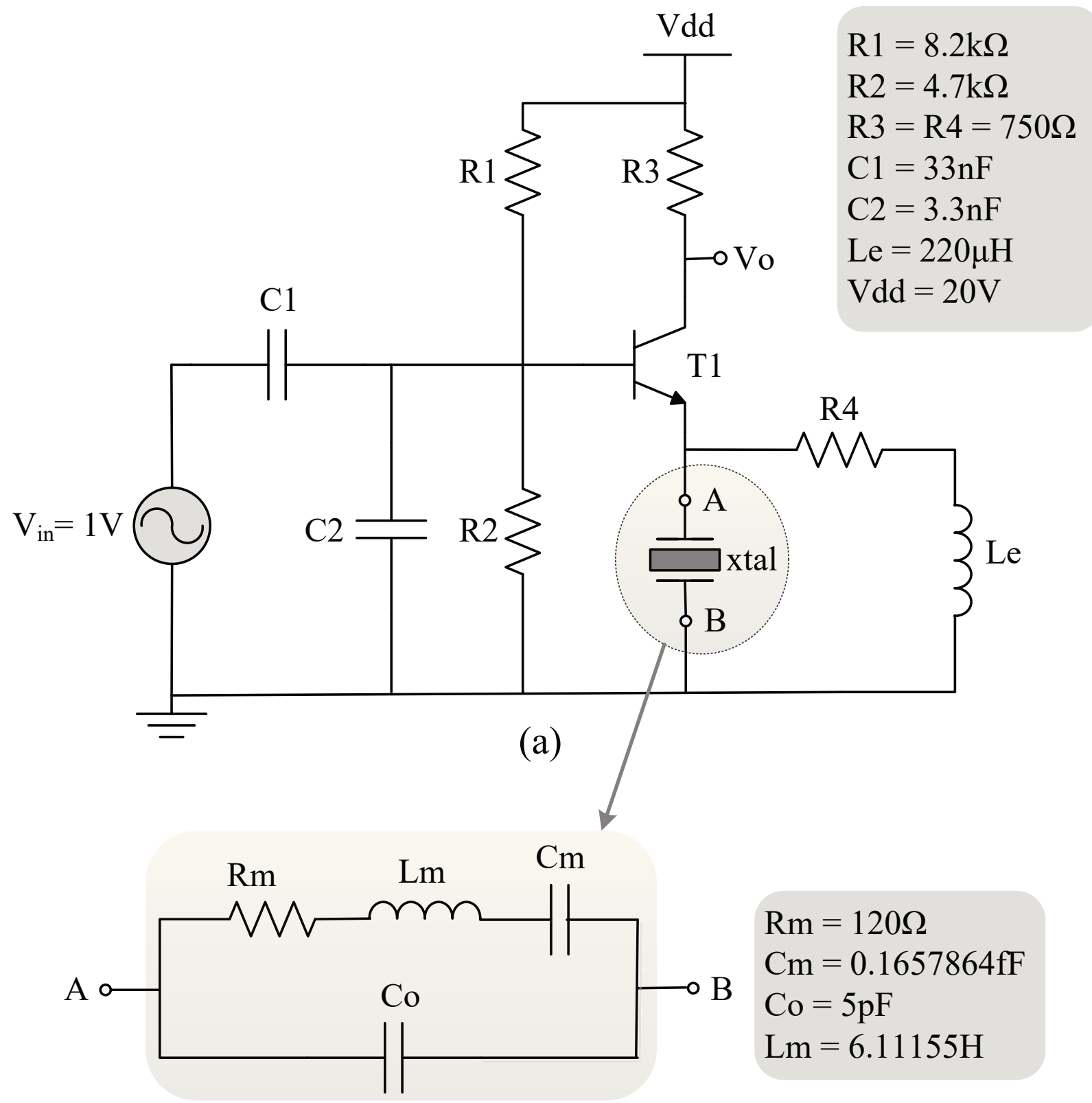

(b)

Figure 2.1: (a) Tuned BJT Amplifier Circuit (Example I), (b) Crystal Equivalent Circuit. 


\section{Chapter 3}

\section{Model Order Reduction Techniques}

\subsection{Introduction}

The Model Order Reduction (MOR) technique has proven to be very efficient in the simulation of Very Large Scale Integrated (VLSI) circuits. MOR is based on a rigorous mathematical algorithm approximating the input to output behavior of a given VLSI circuit by using a much smaller model [12]. As a result, the main CPU time spent to compute the simulation results is significantly reduced while preserving a high level accuracy of the results. The main intent is to allow the Reduced Order Model (ROM) to have enough of a high simulation efficiency. In order to realize this intention, the size of the ROM should not only be small enough, but the result which is achieved from the ROM should have a good fit to the original system simulation. For the linear circuit, the stability and passivity should also be preserved.

For the past twenty years, the research area of the linear system Model Order Reduction has been continually developed. Great achievements and techniques have been published in terms of macromodeling [13].

This chapter presents a review of the well-known projection based MOR techniques, particularly the Krylov subspace and Moment Matching Technique (MMT). 
In addition, the format of the ROM is introduced. Finally, the POD-Galerkin method will be illustrated.

\subsection{Model Order Reduction for Linear Circuits}

The Moment Matching Technique (MMT) is based on the Taylor series expansion to approximate the response of the original circuit $[14,15]$. The MNA equation of a linear circuit in the frequency domain is given in the form

$$
\begin{aligned}
(\mathbf{G}+s \mathbf{C}) \mathbf{X}(s) & =\mathbf{b}(s) \\
\mathbf{b}(s) & =\mathbf{B} \mathbf{U}(s) \\
\mathbf{Y}(s) & =\mathbf{L X}(s)
\end{aligned}
$$

which can also be expressed as

$$
\mathbf{Y}(s)=\mathbf{H}(s) \mathbf{U}(s)
$$

where $\mathbf{X}(s) \in \mathbb{C}^{n}$ is the vector of circuit variables and $\mathbf{H}(s)$ is the linear circuit transfer function in the complex frequency domain. The complex-frequency $s$ is the Laplace integration constant. The circuit transfer function in (3.2) is defined as

$$
\mathbf{H}(s)=\mathbf{L}(\mathbf{G}+s \mathbf{C})^{-1} \mathbf{B}
$$


It can also be equivalently represented as

$$
\begin{aligned}
\mathbf{H}(s) & =\mathbf{L}(\mathbf{I}-s \mathbf{A})^{-1} \mathbf{R} \\
\mathbf{R} & \triangleq \mathbf{G}^{-1} \mathbf{B} \\
\mathbf{A} & \triangleq-\mathbf{G}^{-1} \mathbf{C} .
\end{aligned}
$$

The transfer function could be expanded into the Taylor series with respect to the complex frequency $s$ at zero frequency point $s_{0}=0$ is

$$
\begin{aligned}
\mathbf{H}(s) & =\mathbf{L}\left(\mathbf{R}+\mathbf{A} \mathbf{R} s+\mathbf{A}^{2} \mathbf{R} s^{2}+\cdots+\mathbf{A}^{i} \mathbf{R} s^{i}+\cdots\right) \\
& =\sum_{i=0}^{\infty} \mathbf{L A}^{i} \mathbf{R} s^{i}=\sum_{i=0}^{\infty} \mathbf{L M}_{i} s^{i}
\end{aligned}
$$

where $\mathbf{M}_{i} \triangleq \mathbf{A}^{i} \mathbf{R} \in \mathbb{R}^{n \times p}$ is the $i$ th order (block-) moment of $s_{0}=0$, i.e.,

$$
\mathbf{M}_{0}=\mathbf{R}, \quad \mathbf{M}_{1}=\mathbf{A R}, \quad \mathbf{M}_{2}=\mathbf{A}^{2} \mathbf{R}, \quad \cdots, \quad \mathbf{M}_{i}=\mathbf{A}^{i} \mathbf{R}
$$

From (3.7) the following recursive relationship for the moments can be defined

$$
\mathbf{M}_{i}=\mathbf{A} \mathbf{M}_{i-1}
$$

The subspace, which is constructed by the moments shown in (3.7), is called the Krylov subspace. The Krylov subspace is formally defined as a space spanned by the column vectors in the moment matrix as shown in (3.9).

$$
\mathbf{V}_{m}=\mathcal{K}_{r}(\mathbf{A}, \mathbf{R}, m)=\operatorname{colspan}\left\{\mathbf{R}, \mathbf{A R}, \mathbf{A}^{2} \mathbf{R}, \cdots, \mathbf{A}^{m-1} \mathbf{R}\right\}
$$




\subsection{Krylov-Subspace based Model Order Reduc- tion}

The most successful and commonly used algorithm for reduction of large-scale linear systems is the (block) Krylov-subspace based projection methods. The orthogonal projection matrix $\mathbf{Q} \in \mathbb{R}^{n \times m}$ that maps the $n$-dimensional state-space of original system into a $m$-dimensional subspace is constructed such that its column vectors are the basis spanning the system Krylov-subspace as

$$
\operatorname{colsp}\{\mathbf{Q}\}=\operatorname{colspan}\left\{\mathbf{R}, \mathbf{A R}, \mathbf{A}^{2} \mathbf{R}, \cdots, \mathbf{A}^{m-1} \mathbf{R}\right\}
$$

The Arnoldi algorithm is a numerically efficient iterative method which is commonly used to form the set of orthonormal vectors as a basis for the given Krylov subspace as shown in (3.10) [16]. Among the Arnoldi-based methods, PRIMA [17] is the most common method of choice for the reduction of RLC networks while the original passivity is preserved.

Given the projection matrix $\mathbf{Q}$, a reduced linear system of much smaller order is

derived by a variable change as $\mathbf{X}(s)=\mathbf{Q} \hat{\mathbf{X}}(s)$ and multiplying $\mathbf{Q}^{t}$ from the left to the original system in (3.1). The reduced system is defined as

$$
\begin{aligned}
& (\hat{\mathbf{G}}+s \hat{\mathbf{C}}) \hat{\mathbf{X}}(s)=\hat{\mathbf{B}} \\
& \mathbf{Y}(s) \approx \hat{\mathbf{L}} \hat{\mathbf{X}}(s)
\end{aligned}
$$

where

$$
\begin{aligned}
\hat{\mathbf{G}} \triangleq \mathbf{Q}^{t} \mathbf{G Q} & \hat{\mathbf{C}} \triangleq \mathbf{Q}^{t} \mathbf{C Q} \\
\hat{\mathbf{B}} \triangleq \mathbf{Q}^{t} \mathbf{B} & \hat{\mathbf{L}} \triangleq \mathbf{L Q}
\end{aligned}
$$


The reduced model in (3.11) while preserving the main properties of the original system provides a sufficiently accurate approximation for the system's output $\mathbf{Y}(s)$. Also, it is proved that $\mathbf{Y}(s)$ from the reduced model (3.11) of order $m$ preserves the first $m$ moments of the original network (3.1).

\subsection{POD-Galerkin Model Order Reduction}

The Proper Orthogonal Decomposition (POD) is a well known approach for model order reduction in many applications such as fluid dynamics, aerodynamics, image

processing, and electrical engineering [18-21]. Proper orthogonal decomposition also provides a technique for analyzing multidimensional data. This method can be successfully applied to both linear and nonlinear systems [3] to construct reduced order models using Galerkin projection scheme.

\subsubsection{Introduction of the POD Basis}

POD method provides an efficient means of deriving the reduced basis for highdimensional nonlinear systems resulting in the capture of the greatest possible energy of the system. This process essentially constructs orthonormal projection basis from the snapshots of state-response (/data) vectors $\mathbf{x}(\tau)$ evaluated at $N$ different values for $\tau$, where $\tau$ represents system's parameters, e.g., $\alpha$ and/or time $t$. These samples of response are obtained through simulation for some training input excitation and are collected in the columns of a data matrix as

$$
\mathcal{X}=\left[\mathbf{x}\left(\tau_{1}\right), \mathbf{x}\left(\tau_{2}\right), \ldots, \mathbf{x}\left(t_{N}\right)\right] \in \mathbb{R}^{n \times N}
$$

where generally $N \ll n$. 
The POD basis are calculated through performing a Singular-Value Decomposition (SVD) of the data matrix as

$$
\mathcal{X}=\mathbf{U} \Sigma \mathbf{V}^{t}
$$

The first $m$ columns of $\mathbf{U}$ are the POD basis vectors corresponding to the highest singular values. After obtaining the projection matrix from POD approach, it is used to generate a reduced model via a Galerkin projection scheme. Steps of POD to construct the matrix $\mathbf{Q}$ is shown in Algorithm-3.

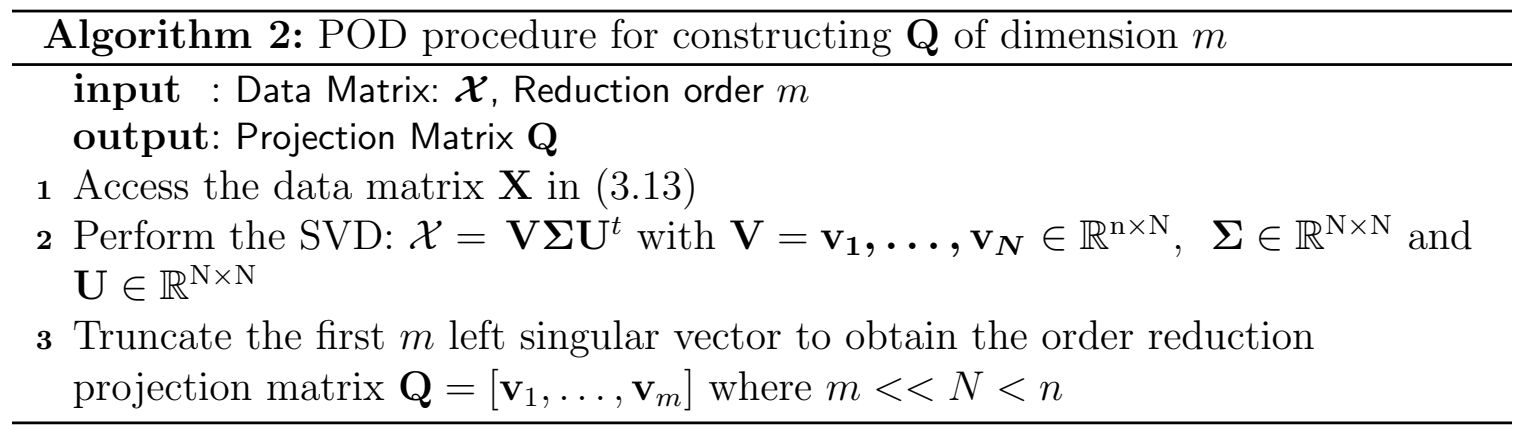

\subsection{Model Order Reduction for Nonlinear Circuits}

Similar to the case of linear systems, nonlinear model order reduction is also to reduce the computational complexity (e.g., time and memory requirement) of large-scale nonlinear dynamic and algebraic systems as given in Equations (2.1) and (2.2), respectively. This is achieved through the approximation of large systems using models of much lower dimension that can produce nearly the same input/output characteristic.

\subsubsection{Projection Framework for Nonlinear MOR}

Almost similar to the case of linear systems, a projection recipe can be applied to the nonlinear system of equations presented in (2.1) and (2.2). 
To further explain the framework, let us consider the nonlinear DC equation (2.2) as presented in the section 2.2 and for the ease of reference is repeated here

$$
\Phi(\mathbf{x})=\mathbf{G x}+\mathbf{f}(\mathbf{x})-\mathbf{b}=0 \in \mathbb{R}^{n}
$$

The Jacobian matrix for the nonlinear algebraic equation in (3.15) is defined as the first-order derivative of the nonlinear algebraic equation in (3.15) with respect to state-variables as

$$
\boldsymbol{\Psi}(\mathbf{x}) \triangleq \frac{\partial \Phi(\mathbf{x})}{\partial \mathbf{x}}=\mathbf{G}+\mathbf{J}(\mathbf{x})
$$

where

$$
\mathbf{J}(\mathbf{x}) \triangleq \frac{\partial \mathbf{f}(\mathbf{x})}{\partial \mathbf{x}}
$$

Applying the projection recipe to (3.15) leads to the following reduced system of equations,

$$
\mathbf{Q}^{t} \mathbf{\Phi}(\mathbf{Q} \hat{\mathbf{x}}) \mathbf{Q}^{t} \mathbf{G Q} \hat{\mathbf{x}}+\mathbf{Q}^{t} \mathbf{f}(\mathbf{Q} \hat{\mathbf{x}})-\mathbf{Q}^{t} \mathbf{b}=0 \in \mathbb{R}^{m}
$$

This can be equivalently rewritten as

$$
\hat{\Phi}(\hat{\mathbf{x}})=\hat{\mathbf{G}} \hat{\mathbf{x}}+\hat{\mathbf{f}}(\hat{\mathbf{x}})-\hat{\mathbf{b}}=0
$$

where in absence of analytical forms, the reduced nonlinear functions are only symbolically defined as

$$
\begin{gathered}
\hat{\mathbf{\Phi}}(\hat{\mathbf{x}}) \triangleq \mathrm{Q}^{t} \mathbf{\Phi}(\mathbf{Q} \hat{\mathbf{x}}) \\
\hat{\mathbf{f}}(\hat{\mathbf{x}}) \triangleq \mathbf{Q}^{t} \mathbf{f}(\mathbf{Q} \hat{\mathbf{x}})
\end{gathered}
$$


The corresponding Jacobian for the reduced system is defined as

$$
\hat{\mathbf{\Psi}}(\hat{\mathbf{x}})=\mathbf{Q}^{t} \mathbf{G Q}+\mathbf{Q}^{t} \mathbf{J}(\mathbf{Q} \hat{\mathbf{x}}) \mathbf{Q} \in \mathbb{R}^{m \times m}
$$

It is to be noted that, selection of the reduced basis can directly effect the accuracy of the resulting reduced models.

\subsubsection{Challenges of Projection Framework}

The projection-based approach has been the most successful algorithms for reduction of large-scale systems. However, application of the projection-based dimension reduction approaches to solve nonlinear large systems in (2.1) and (2.2) can generally face some computational challenges. This occurs due to the CPU-expense associated with the evaluation of the highly nonlinear in reduced models.

In the general case, interpreting the terms $\mathbf{Q}^{t} \mathbf{f}(\mathbf{Q} \hat{\mathbf{x}})$ and $\mathbf{Q}^{t} \mathbf{J}(\mathbf{Q} \hat{\mathbf{x}}) \mathbf{Q}$ (in (3.20) and (3.21), respectively) as reduced models is problematic. Since $\mathbf{f}(\mathbf{x})$ and $\mathbf{J}(\mathbf{x})$ are nonlinear function and the only way to computing $\mathbf{Q}^{t} \mathbf{f}(\mathbf{Q} \hat{\mathbf{x}})$ and $\mathbf{Q}^{t} \mathbf{J}(\mathbf{Q} \hat{\mathbf{x}}) \mathbf{Q}$ is to (a) directly construct $\mathbf{x}=\mathbf{Q} \hat{\mathbf{x}}$ then, (b) explicitly evaluate $\mathbf{f}(\mathbf{x})$ and $\mathbf{J}(\mathbf{x})$, and (c) compute $\mathbf{Q}^{t} \mathbf{f}(\mathbf{Q} \hat{\mathbf{x}})$ and $\mathbf{Q}^{t} \mathbf{J}(\mathbf{Q} \hat{\mathbf{x}}) \mathbf{Q}$. All these computationally cumbersome tasks need to be performed in every N-R iterations. As a result, an efficient simulation is not guaranteed. Thus, regardless of the reduction in a size of the nonlinear systems, the computational cost to evaluate the projected-nonlinear function and its Jacobian still depend on the original dimension and hence, the simulation times hardly lead to an improvement over the original systems.

To avoid this issue, the approximated approach based on linearization such as piecewise linear techniques [22-24] are initially used to perform the nonlinear simulation. Despite the devising simplicity of the idea, this class of methods in general 
suffers from the following issues

(a) high model generation time

(b) heuristic-based linearization-points selection

(c) limited accuracy, generally inferior to the projection-based methods

(d) very poor approximation for the large signal behavior

For the cases of that, model generation time and accuracy are highly important factors using a projection-based reduction method such as POD will be inevitable.

\subsubsection{POD-Galerkin Complexity Problem}

This section illustrates the computational-cost analysis for solving a nonlinear reduced system using POD-Galerkin approach.

First, the computational complexity for evaluating the following term

$$
\hat{\mathbf{f}}(\hat{\mathbf{x}})=\underbrace{\mathbf{Q}^{t}}_{m \times n} \underbrace{\mathbf{f}(\mathbf{Q} \hat{\mathbf{x}})}_{n \times 1}
$$

includes the costs for two matrix-to-vector multiplications, i.e., $\mathcal{O}(4 n \mathrm{~m})$ and for evaluating a full nonlinear function $\mathbf{f}$ is $\mathcal{O}(\alpha(n))$, where $\alpha$ is some function of $n$. Then, the total complexity for $(3.22)$ is about $\mathcal{O}(\alpha(n)+4 n m)$ at each iteration.

Second, the Jacobian of the nonlinear function $\hat{\Psi}(\hat{\mathbf{x}})$ in $(3.21)$ must be computed at each iteration with a computational cost that also depends on the original-system dimension $n$ as

$$
\hat{\mathbf{\Psi}}(\hat{\mathbf{x}})=\underbrace{\mathrm{Q}^{t}}_{m \times n} \underbrace{\mathrm{G}}_{n \times n} \underbrace{\mathrm{Q}}_{n \times m}+\underbrace{\mathrm{Q}^{t}}_{m \times n} \underbrace{\mathbf{J}(\mathrm{Q} \hat{\mathbf{x}})}_{n \times n} \underbrace{\mathrm{Q}}_{n \times m}
$$

where $\mathbf{Q}^{t} \mathbf{G Q}$ is precomputed. The computational complexity for computing (3.23) at each iteration is roughly $\mathcal{O}\left(m^{2}+\alpha(n)+2 n^{2} m+2 n m^{2}+2 n m\right)$ if $\mathbf{J}$ is dense. The $2 n^{2} m$ term becomes $\mathcal{O}(n m)$ if $\mathbf{J}$ is sparse or diagonal [3]. 
The techniques to overcome the explained computational inefficiency in the projection-based methods through approximating nonlinear functions are introduced in the following chapters. 


\section{Chapter 4}

\section{Nonlinear Model Order Reduction for DC Solution}

As discussed in Chapter 2, solving the DC operating point of the nonlinear circuit is an important task. The Newton-Raphson method in the SPICE simulator is employed to do it, whose convergence could be guaranteed under the condition that initial approximation is close enough to the solution. Continuation methods are used to overcome this non-convergence problem of the Newton-Raphson method.

A version of the continuation method based on the source stepping was also reviewed in Section 2.3. While the source-ramping recipe increases the probability of convergence for Newton-Raphson iterations, repetitive simulations of the large nonlinear circuits in the original sizes still remain a burdensome task in DC analysis.

This chapter presents fundamentals of a method for efficient DC analysis of com-

plex nonlinear circuits. It combines continuation methods with model reduction techniques using Krylov subspace technique. It is based on forming the basis for the Krylov subspace spanned by the moments of the nonlinear function with respect to the parameter. Then, the reduced order system is obtained by projecting the set of nonlinear equations, whose solution represents the DC operating point, into a 
subspace of a much lower dimension leading to a significant reduction in the computational expense.

Details of the algorithm are presented briefly in the following sections.

\subsection{Computation of the Moments in the Taylor Series Expansions}

In this section, the computation of derivatives with respect to the sweeping parameter is presented in the close form. Similar to the Krylov-subspace based MOR algorithm introduced in Chapter 3, it is needed to derive the moments of parametrized-response (with respect to the circuit parameter) from the augmented equation (2.8). Eq. (2.8) is repeated here for the ease of reference

$$
\mathbf{\Phi}(\mathbf{x}, \alpha)=\mathbf{G x}+\mathbf{f}(\mathbf{x})-\alpha \mathbf{b}=0
$$

The solution of $(4.1) \mathbf{x}(\alpha)$ is an implicit function of the sweeping parameter $\alpha$. Hence, it can be expanded in a Taylor series at $\alpha=\alpha_{0}$ as

$$
\mathbf{x}(\alpha)=\sum_{i=0} \mathbf{M}_{i}\left(\alpha-\alpha_{0}\right)^{i}
$$

where the $i^{\text {th }}$ moment $\mathbf{M}_{i}$ is the normalized derivative evaluated at $\alpha_{0}$ defined as

$$
\left.\mathbf{M}_{i} \triangleq \frac{1}{i !} \frac{d^{i} \mathbf{x}(\alpha)}{d \alpha^{i}}\right|_{\alpha=\alpha_{0}}
$$

In a similar fashion, the Taylor Series expansion for the nonlinear function in (4.1) 
$\mathbf{f}(\mathbf{x}(\alpha))$ at the point $\alpha_{0}$ is

$$
\mathbf{f}(\mathbf{x}(\alpha))=\sum_{i=0} \mathbf{F}_{i}\left(\alpha-\alpha_{0}\right)^{i}
$$

where the $i^{\text {th }}$ moment of nonlinear function $\mathbf{F}_{i}$ is the normalized derivative evaluated at $\alpha_{0}$ defined as

$$
\left.\mathbf{F}_{i} \triangleq \frac{1}{i !} \frac{d^{i} \mathbf{f}(\mathbf{x}(\alpha))}{d \alpha^{i}}\right|_{\alpha=\alpha_{0}}
$$

By substituting (4.4) and (4.5) in (4.1), we get

$$
\mathbf{G}\left(\sum_{i=0} \mathbf{M}_{i}\left(\alpha-\alpha_{0}\right)^{i}\right)+\sum_{i=0} \mathbf{F}_{i}\left(\alpha-\alpha_{0}\right)^{i}-\mathbf{b}\left(\alpha-\alpha_{0}\right)-\mathbf{b} \alpha_{0}=0
$$

Ensuring the equality in (4.6) for any value of $\alpha$ requires that for $i=1,2, \ldots$, there is

$$
\begin{array}{cc}
\mathbf{G M}_{0}+\mathbf{F}_{0}-\alpha_{0} \mathbf{b}=0, & i=0 \\
\mathbf{G M}_{1}+\mathbf{F}_{1}-\mathbf{b}=0, & i=1 \\
\vdots & \vdots \\
\mathbf{G M}_{i}+\mathbf{F}_{i}=0, & i \geq 2
\end{array}
$$

Obtaining a closed-form representation for parametrized-solution of the augmented system $\mathbf{x}(\alpha)$, is theoretically possible by finding (the sufficient number of) its leading moments $\mathbf{M}_{i}$ in (4.2) through solving Equations (4.7)-(4.9).

The zero-th moment $\mathbf{M}_{0}$ which is the evaluation of response at the expansion point $\mathbf{M}_{0}=\left.\mathbf{x}(\alpha)\right|_{\alpha=\alpha_{0}}$ can be directly found by applying $\mathrm{N}-\mathrm{R}$ to (4.7). It is given that the nonlinear function is known analytically from MNA formulation and its zeroth moment is $\mathbf{F}_{0}=\left.\mathbf{f}(\mathbf{x}(\alpha))\right|_{\alpha=\alpha_{0}}=\mathbf{f}\left(\mathbf{M}_{0}\right)$. For the special case, where $\alpha_{0}=0$, in 
absence of the source term in (4.6), the zero-th moments are trivially $\mathbf{M}_{0}=0$ and $\mathbf{F}_{0}=0$.

To find the higher moments $\mathbf{M}_{i}$ for $i=1,2, \ldots$, the derivatives of nonlinear function is needed.

\subsubsection{Evaluation of the Moments for Nonlinear Function}

When forming the circuit MNA equations, the nonlinear function $\mathbf{f}(\mathbf{x})$ is identified in

closed form. Hence, its moments (4.5) as the coefficient of its Taylor series expansion at $\alpha_{0}=0(4.4)$ can be readily evaluated at $\alpha=0$.

\section{- Evaluation of the First-Moment for Nonlinear Function}

In particular, the first moment of $\mathbf{f}(\mathbf{x})$, i.e., the first derivative of the function with respect to $\alpha$ evaluated at $\alpha_{0}=0$ can be obtained as presented below.

$$
\begin{aligned}
\frac{d \mathbf{f}(\mathbf{x}(\alpha))}{d \alpha} & =\frac{d \mathbf{f}(\mathbf{x})}{d \mathbf{x}} \times \frac{d \mathbf{x}}{d \alpha}=\mathbf{J}(\mathbf{x}) \times \frac{d \mathbf{x}}{d \alpha} \\
& =\mathbf{J}\left(\sum_{i=0} \mathbf{M}_{i} \alpha^{i}\right) \times \frac{d}{d \alpha} \sum_{i=0} \mathbf{M}_{i} \alpha^{i} \\
& =\mathbf{J}\left(\sum_{i=0} \mathbf{M}_{i} \alpha^{i}\right) \sum_{i=1} i \mathbf{M}_{i} \alpha^{i-1} \\
& =\boldsymbol{\zeta}(\alpha) \sum_{i=1}^{\infty} i \mathbf{M}_{i} \alpha^{i-1}
\end{aligned}
$$

where Jacobian as an explicit function of $\alpha$ is denoted by $\zeta(\alpha)$, i.e.,

$$
\boldsymbol{\zeta}(\alpha) \triangleq \mathbf{J}(\mathbf{x}(\alpha))=\mathbf{J}\left(\sum_{i=0} \mathbf{M}_{i} \alpha^{i}\right)
$$

By evaluating the derivative in (4.10) at $\alpha=0$, the first moment of the nonlinear 
function will be

$$
\mathbf{F}_{1}=\left.\frac{d \mathbf{f}(\mathbf{x}(\alpha))}{d \alpha}\right|_{\alpha=0}=\mathbf{J}\left(\mathbf{M}_{0}\right) \mathbf{M}_{1}=\boldsymbol{\zeta}(0) \mathbf{M}_{1}
$$

\section{- Evaluation of the Second- and Higher-Moments for Nonlinear Function}

Considering the first derivative in (4.10) as a multiplication of two functions of $\alpha$,

$$
\begin{aligned}
\frac{d \mathbf{f}(\mathbf{x}(\alpha))}{d \alpha} & =\mathbf{J}(\mathbf{x}(\alpha)) \times \frac{d \mathbf{x}(\alpha)}{d \alpha}=\mathbf{J}\left(\sum_{i=0} \mathbf{M}_{i} \alpha^{i}\right) \times \sum_{i=1} i \mathbf{M}_{i} \alpha^{i-1} \\
& =\underbrace{\boldsymbol{\zeta}(\alpha)}_{\mathbf{u}(\alpha)} \times \underbrace{\sum_{i=1} i \mathbf{M}_{i} \alpha^{i-1}}_{\mathbf{v}(\alpha)}
\end{aligned}
$$

such that they are continuously-differentiable (taken to be $\mathcal{C}^{i}$ ) then, the $i^{\text {th }}$ derivatives of their product can be obtained using the following "Leibnitz's theorem".

Theorem 1 (Leibnitz Theorem). Let $u(\alpha)$ and $v(\alpha)$ be functions with continuous $m^{\text {th }}$ derivative. Then their product is also $m$-continues $\left(\mathcal{C}^{m}\right)$, and

$$
\frac{d^{m}}{d \alpha^{m}}[u(\alpha) v(\alpha)]=\sum_{r=0}^{m}\left(\begin{array}{c}
m \\
r
\end{array}\right) \frac{d^{r}}{d \alpha^{r}}[u(\alpha)] \frac{d^{m-r}}{d \alpha^{m-r}}[v(\alpha)]
$$

where

$$
\left(\begin{array}{c}
m \\
r
\end{array}\right)=\frac{m !}{r !(m-r) !}
$$

is the usual binomial coefficient [25].

In (4.5), the $m^{\text {th }}$ order derivative of the nonlinear function is found using the 
Leibnitz theorem at point $\alpha=0$, leading to the following form for the $m^{\text {th }}$ moment.

$$
\begin{gathered}
\mathbf{F}_{m}=\left.\frac{1}{m !} \frac{d^{m} \mathbf{f}(\mathbf{x}(\alpha))}{d \alpha^{m}}\right|_{\alpha=0}=\left.\frac{1}{m !}\left(\frac{d^{m-1}}{d \alpha^{m-1}}\left[\frac{d \mathbf{f}(\mathbf{x}(\alpha))}{d \alpha}\right]\right)\right|_{\alpha=0}= \\
\left.\frac{1}{m !}\left(\frac{d^{m-1}}{d \alpha^{m-1}}\left[\mathbf{J}\left(\sum_{i=0} \mathbf{M}_{i} \alpha^{i}\right) \times \sum_{i=1} i \mathbf{M}_{i} \alpha^{i-1}\right]\right)\right|_{\alpha=0}= \\
\left.\frac{1}{m !}\left(\frac{d^{m-1}}{d \alpha^{m-1}}[\underbrace{\boldsymbol{\zeta}(\alpha)}_{\mathbf{u}(\alpha)} \times \underbrace{\sum_{i=1} \mathbf{M}_{i} \alpha^{i-1}}_{\mathbf{v}(\alpha)}]\right)\right|_{\alpha=0}= \\
\left.\frac{1}{m !}\left(\sum_{r=0}^{m-1}\left(\begin{array}{c}
m-1 \\
r
\end{array}\right)\left[\frac{d^{r}}{d \alpha^{r}}[\boldsymbol{\zeta ( \alpha )}] \times \frac{d^{m-r-1}}{d \alpha^{m-r-1}}\left[\sum_{i=1} i \mathbf{M}_{i} \alpha^{i-1}\right]\right]\right)\right|_{\alpha=0}= \\
\left.\frac{1}{m !}\left(\sum_{r=0}^{m-1}\left(\begin{array}{c}
m-1 \\
r
\end{array}\right)\left[\frac{d^{r}}{d \alpha^{r}}[\boldsymbol{\zeta}(\alpha)] \times \frac{d^{m-r-1}}{d \alpha^{m-r-1}}\left[\mathbf{M}_{1}+2 \mathbf{M}_{2} \alpha+3 \mathbf{M}_{3} \alpha^{2}+\cdots\right]\right]\right)\right|_{\alpha=0}
\end{gathered}
$$


By calculating the derivatives in (4.16), we get

$$
\begin{aligned}
& \mathbf{F}_{m}=\frac{1}{m !} \times( \\
& \left.\left(\boldsymbol{\zeta}(\alpha) \times\left(m ! \mathbf{M}_{m}+(m+1) ! \mathbf{M}_{m+1} \alpha+\cdots\right)\right)\right|_{\alpha=0}+ \\
& \left.(m-1)\left(\boldsymbol{\zeta}^{(1)}(\alpha) \times\left((m-1) ! \mathbf{M}_{m-1}+m ! \mathbf{M}_{m} \alpha+\cdots\right)\right)\right|_{\alpha=0}+ \\
& \left.\frac{(m-1)(m-2)}{2 !}\left(\boldsymbol{\zeta}^{(2)}(\alpha) \times\left((m-2) ! \mathbf{M}_{m-2}+(m-1) ! \mathbf{M}_{m-1} \alpha+\cdots\right)\right)\right|_{\alpha=0}+ \\
& \left.\frac{(m-1)(m-2)(m-3)}{3 !}\left(\boldsymbol{\zeta}^{(3)}(\alpha) \times\left((m-3) ! \mathbf{M}_{m-3}+(m-2) ! \mathbf{M}_{m-2} \alpha+\cdots\right)\right)\right|_{\alpha=0}+ \\
& \left.+\left.\left(\boldsymbol{\zeta}^{(m-1)}(\alpha)\left(\mathbf{M}_{1}+2 \mathbf{M}_{2} \alpha+3 \mathbf{M}_{3} \alpha^{2}+\cdots\right)\right)\right|_{\alpha=0}\right)
\end{aligned}
$$

It can be also further simplified as

$$
\begin{aligned}
\mathbf{F}_{m}= & \frac{1}{m !} \times\left(m ! \boldsymbol{\zeta}(0) \mathbf{M}_{m}+(m-1)(m-1) ! \boldsymbol{\zeta}^{(1)}(0) \mathbf{M}_{m-1}+\right. \\
& \frac{(m-1)(m-2)(m-2) !}{2} \boldsymbol{\zeta}^{(2)}(0) \mathbf{M}_{m-2}+ \\
& \left.\frac{(m-1)(m-2)(m-3)(m-3) !}{3} \boldsymbol{\zeta}^{(3)}(0) \mathbf{M}_{m-3}+\cdots+\boldsymbol{\zeta}^{(m-1)}(0) \mathbf{M}_{1}\right)
\end{aligned}
$$


and then,

$$
\begin{array}{r}
\mathbf{F}_{m}=\boldsymbol{\zeta}(0) \mathbf{M}_{m}+\frac{(m-1)}{m} \boldsymbol{\zeta}^{(1)}(0) \mathbf{M}_{m-1}+\frac{(m-2)}{2 ! m} \boldsymbol{\zeta}^{(2 !)}(0) \mathbf{M}_{m-2}+ \\
\frac{(m-3)}{3 ! m} \boldsymbol{\zeta}^{(3 !)}(0) \mathbf{M}_{m-3}+\cdots+\frac{1}{m !} \boldsymbol{\zeta}^{(m-1)}(0) \mathbf{M}_{1}
\end{array}
$$

From (4.19), it is concluded that

$$
\mathbf{F}_{m}=\sum_{r=0}^{m-1} \frac{(r+1)}{m(m-r-1) !} \boldsymbol{\zeta}^{(m-r-1)}(0) \mathbf{M}_{r+1}, \quad \text { for } m \geq 2
$$

where

$$
\boldsymbol{\zeta}^{(i)}(0)=\left.\frac{d^{i}}{d \alpha^{i}} \boldsymbol{\zeta}(\alpha)\right|_{\alpha=0} \in \mathbb{R}^{n \times n}
$$

and

$$
\boldsymbol{\zeta}^{(0)}(0)=\left.\boldsymbol{\zeta}(\alpha)\right|_{\alpha=0}=\left.\mathbf{J}(\mathbf{x}(\alpha))\right|_{\alpha=0)}=\mathbf{J}(\mathbf{x}(0))=\mathbf{J}\left(\mathbf{M}_{0}\right) \in \mathbb{R}^{n \times n}
$$

In the rest of this thesis, for the sake of notation abbreviation $\mathbf{J}_{0}$ will be used to represent $\mathbf{J}(\mathbf{x}(0))=\mathbf{J}\left(\mathbf{M}_{0}\right)$.

\subsubsection{Evaluation of the Moments for DC Solution}

The moments for DC solution $\mathbf{x}(\alpha)$ (4.3) are evaluated as the coefficient for its Taylor series expansion at $\alpha_{0}=0$ (4.2). The steps of computation are presented as follows. 


\section{- Computation of the zero-th moment for DC solution:}

The zero-th moment of the DC response at the expansion point $\mathbf{M}_{0}=\mathbf{x}\left(\alpha_{0}\right)$ and the zero-th moment of nonlinear function $\mathbf{F}_{0}=\mathbf{f}\left(\alpha_{0}\right)$ need to be obtained to satisfy (4.7). In cases that, $\alpha_{0}=0$ has been selected as the expansion-point, considering that the source term in $(4.1)$ is zero $(\alpha \mathbf{b}=0)$, it is straightforward to see that

$$
\mathbf{M}_{0}=\left.\mathbf{x}(\alpha)\right|_{\alpha=0}=\mathbf{x}(0)=0
$$

and accordingly,

$$
\mathbf{F}_{0}=\left.\mathbf{f}(\mathbf{x}(\alpha))\right|_{\alpha=0}=\mathbf{f}(\mathbf{x}(0))=\mathbf{f}(0)=0
$$

- Computation of the first moment for DC solution:

By substituting (4.12) in (4.8), we have

$$
\mathrm{GM}_{1}+\mathbf{F}_{1}=\mathrm{GM}_{1}+\boldsymbol{\zeta}(0) \mathbf{M}_{1}=\mathbf{b}
$$

and hence,

$$
(\mathbf{G}+\boldsymbol{\zeta}(0)) \mathbf{M}_{1}=\mathbf{b}
$$

Solution of (4.26) computationally requires one LU decomposition of $(\mathbf{G}+\boldsymbol{\zeta}(0))$ and one forward/backward substitution.

\section{- Computation of the second- and higher-moments for DC solution:}

These moments are computed through combining (4.20) and (4.9) as presented below. ○ Second-Order Moment, $m=2$ : 
First, to serve the illustration purpose, the detailed computation of the secondorder moment is presented as shown below. From (4.9) we have

$$
\mathrm{GM}_{2}+\mathbf{F}_{2}=0
$$

where

$$
\begin{aligned}
\mathbf{F}_{2}=\sum_{r=0}^{1} \frac{(r+1)}{2(1-r) !} \boldsymbol{\zeta}^{(1-r)}(0) \mathbf{M}_{r+1} & =\frac{1}{2} \boldsymbol{\zeta}^{(1)}(0) \mathbf{M}_{1}+\boldsymbol{\zeta}^{(0)}(0) \mathbf{M}_{2} \\
& =\frac{1}{2} \boldsymbol{\zeta}(0)^{(1)} \mathbf{M}_{1}+\boldsymbol{\zeta}(0) \mathbf{M}_{2}
\end{aligned}
$$

By substituting (4.28) in (4.27), we get

$$
(\mathbf{G}+\boldsymbol{\zeta}(0)) \mathbf{M}_{2}=\underbrace{-\frac{1}{2} \boldsymbol{\zeta}(0)^{(1)} \mathbf{M}_{1}}_{\triangleq b_{2}}
$$

○ Higher-Order Moment, $m>2$ :

$$
\mathbf{F}_{m}=\boldsymbol{\zeta}(0) \mathbf{M}_{m}+\sum_{r=0}^{m-2} \frac{(r+1)}{m(m-r-1) !} \boldsymbol{\zeta}^{(m-r-1)}(0) \mathbf{M}_{r+1}
$$

By substituting (4.31) in (4.9), we get

$$
(\mathbf{G}+\boldsymbol{\zeta}(0)) \mathbf{M}_{m}=\underbrace{-\sum_{r=0}^{m-2} \frac{(r+1)}{m(m-r-1) !} \boldsymbol{\zeta}^{(m-r-1)}(0) \mathbf{M}_{r+1}}_{\triangleq b_{m}}
$$

It is to be noted that, from (4.31), the $m^{\text {th }}$ moment of the DC solution with respect to $\alpha$ is found from the lower-order moments $\mathbf{M}_{k}$ and $\boldsymbol{\zeta}^{(k)}(0)$ for $k=0, \ldots, m-$ 1. The computational cost required to find $m$ moments is limited to only one LU 
decomposition of a sparse matrix of order $n \times n$ and $m$ forward/backward substitution.

\subsubsection{Summary for Moments}

$$
\left\{\begin{array}{l}
\mathbf{M}_{0}=0 \\
\left(\mathbf{G}+\mathbf{J}_{0}\right) \mathbf{M}_{1}=\mathbf{b} \\
(\mathbf{G}+\boldsymbol{\zeta}(0)) \mathbf{M}_{2}=-\frac{1}{2} \boldsymbol{\zeta}(0)^{(1)} \mathbf{M}_{1} \\
(\mathbf{G}+\boldsymbol{\zeta}(0)) \mathbf{M}_{m}=-\sum_{r=0}^{m-2} \frac{(r+1)}{m(m-r-1) !} \boldsymbol{\zeta}^{(m-r-1)}(0) \mathbf{M}_{r+1}, m>2
\end{array}\right.
$$

As defined in (4.11), it is

$$
\boldsymbol{\zeta}(\alpha) \triangleq \mathbf{J}(\mathbf{x}(\alpha)) \text { and } \quad \boldsymbol{\zeta}^{(i)}(\alpha) \triangleq \mathbf{J}_{i}(\mathbf{x}(\alpha))
$$

As an example of the calculation of the nonlinear function derivatives, consider the exponential function:

$$
f(v)=I_{s}\left(e^{\left(v / V_{T}\right)}-1\right)
$$

The expansion of the exponential gives the result of:

Table 4.1: Summary

\begin{tabular}{|l|l|}
\hline Function & $v=\sum_{i=0} M_{i} \alpha^{i}, \quad f(v)=\sum_{i=0} F_{i} \alpha^{i}$ \\
\hline \hline$f(v)=I_{s}\left(e^{\left(v / V_{T}\right)}-1\right)$ & $F_{0}=I_{s}\left(e^{M_{0} / V_{T}}-1\right)$ \\
& $F_{i}=\frac{1}{i V_{T}} \sum_{r=0}^{i-1} F_{r} M_{i-r}(i-r)+\frac{I_{s}}{V_{T}} M_{i}$ \\
\hline
\end{tabular}




\subsection{Reduced Model via Congruent Transforma- tion}

Following the nonlinear order-reduction paradigm, the DC equation in (4.1) is reduced to a smaller set of unknowns through a congruent projection scheme. The projection

matrix is obtained as (orthogonal) basis for the Krylov (/moments) subspace spanned by the moments of DC solution with respect to sweep parameter,

$$
\mathcal{K} \boldsymbol{r}=\operatorname{span}\left\{\mathbf{M}_{1}, \mathbf{M}_{2}, \ldots, \mathbf{M}_{m}\right\} \quad \text { where } m \ll n
$$

Any orthogonal decomposition such as Singular-Value Decomposition (SVD) or QR can properly serve this purpose. QR is the method of choice in this work to obtain matrix $\mathbf{Q} \in \mathbb{R}^{n \times m}$ as

$$
\left\{\mathbf{M}_{1}, \mathbf{M}_{2}, \ldots, \mathbf{M}_{m}\right\}=\mathbf{Q R}
$$

where $m$ is the order of reduced system $(m \ll n)$, and $\mathbf{Q}^{t} \mathbf{Q}=\mathbf{U}_{m}$ where $\mathbf{U}_{m}$ is an identity matrix. By projecting the DC variables to a lower-dimensional space as $\mathbf{x}=\mathbf{Q} \hat{\mathbf{x}}$ the original equations (4.1) is reduced to the following form

$$
\hat{\mathbf{\Phi}}(\hat{\mathbf{x}}, \alpha)=\hat{\mathbf{G}} \hat{\mathbf{x}}+\hat{\mathbf{f}}(\hat{\mathbf{x}})-\alpha \hat{\mathbf{b}}=0
$$

where

$$
\begin{aligned}
& \hat{\mathbf{G}}=\mathbf{Q}^{t} \mathbf{G Q} \\
& \hat{\mathbf{f}}(\hat{\mathbf{x}})=\mathbf{Q}^{t} \mathbf{f}(\mathbf{Q} \hat{\mathbf{x}}) \\
& \hat{\mathbf{b}}=\mathbf{Q}^{t} \mathbf{b} \\
& \mathbf{x}=\mathbf{Q} \hat{\mathbf{x}}
\end{aligned}
$$


The reduced system in (4.36) due to its much smaller size can be efficiently solved with a much less CPU-cost compare to the original DC equations.

It is to be noted that, the evaluation of nonlinear vector function and the Jacobian matrix for reduced model still need to be repeated in the steps of Newton-Raphson process. As it was previously explained this still remains as a serious bottle-neck, seriously limiting the efficiency of the method. In the following chapters, a method is presented to address this issue and improve the simulation speed. 


\section{Chapter 5}

\section{Review of Nonlinear Model Reduction via Discrete Empirical Interpolations (DEIM)}

As previously highlighted in Chapter 3, it is crucial to improve the efficiency of projection-based MOR methods for nonlinear circuit reduction. It is empirically accepted as a thumb-rule in the MOR community that, the speed-up from using projection-based nonlinear ROMs is generally limited to 2-3. Hence, improving the simulation time for nonlinear reduced models has high practical importance in the industrial-grade simulations.

Also, it was introduced in Section 3.4 that, among the existing approaches, the proper orthogonal decomposition (POD) method provides an efficient means of deriving the reduced basis for high-dimensional nonlinear systems. This technique provides a low-dimensional description of the dynamic system in an optimal way to capture the greatest possible energy of the system.

The mathematical foundation of discrete empirical interpolation method was originally introduced to facilitate the solution of partial differential equations [4]. Later in different disciplines of science and engineering, DEIM attracted attention as an effi-

cient means to overcome the complexity issue associated with the solution of the large 
and complex nonlinear equations [26-28]. Recently in [3], a the POD-DEIM is introduced based on replacing the orthogonal projection of POD with an Petrov-Galerkin (oblique) interpolatory projector.

This chapter introduces DEIM as an effective approach to enhance the CPU gain in the simulation of the nonlinear reduced models. To this end, POD is considered as the reduction method of choice to illustrate and establish the concept.

\subsection{Problem Formulation}

Let the reduced nonlinear dynamic equations and their counterpart DC (algebraic/static) equations be considered as given in (5.1) and (5.2), respectively.

$$
\begin{gathered}
\hat{\mathbf{G}} \hat{\mathbf{x}}(t)+\hat{\mathbf{C}} \frac{d \hat{\mathbf{x}}(t)}{d t}+\hat{\mathbf{f}}(\hat{\mathbf{x}}(t))=\hat{\mathbf{B u}}(t), \quad \mathbf{y}(t)=\hat{\mathbf{L}} \hat{\mathbf{x}}(t) \\
\hat{\mathbf{G}} \hat{\mathbf{x}}(\tau)+\hat{\mathbf{f}}(\hat{\mathbf{x}}(\tau))=\hat{\mathbf{B}} \mathbf{u}_{\mathrm{DC}},
\end{gathered}
$$

where $\tau$ denotes the system's parameters and

$$
\begin{array}{ll}
\hat{\mathbf{C}} \triangleq \hat{\mathbf{Q}}^{t} \mathbf{C} \hat{\mathbf{Q}}, & \hat{\mathbf{G}} \triangleq \hat{\mathbf{Q}}^{t} \hat{\mathbf{C}} \hat{\mathbf{Q}}, \\
\hat{\mathbf{B}} \triangleq \hat{\mathbf{Q}}^{t} \hat{\mathbf{B}}, \text { and } & \hat{\mathbf{L}} \triangleq \hat{\mathbf{L}} \hat{\mathbf{Q}} .
\end{array}
$$

The (so-called) reduced nonlinear function is given as

$$
\hat{\mathbf{f}}(\hat{\mathbf{x}}) \triangleq \mathrm{Q}^{t} \mathbf{f}(\mathbf{Q} \hat{\mathbf{x}})
$$

whose Jacobian is

$$
\hat{\mathbf{J}}(\hat{\mathbf{x}})=\frac{\partial \hat{\mathbf{f}}(\hat{\mathbf{x}})}{\partial \hat{\mathbf{x}}}=\mathbf{Q}^{t} \mathbf{J}(\mathbf{Q} \hat{\mathbf{x}}) \mathbf{Q}
$$


Solving the nonlinear reduced equations in (5.1) and (5.2) to obtain approximate solutions for the original systems $\mathbf{x} \approx \mathrm{Q} \hat{\mathbf{x}}$ requires repetitive evaluation of the reduced nonlinear function in (5.5) as well as its Jacobian (5.5).

\subsection{Formulation of DEIM}

Development of DEIM is mainly based on the following key ideas,

(a) Using two different sets of projection bases (obtained from POD):

(i) the first basis are for the state variables $\mathbf{x}(\tau)$,

(ii) the second basis are for the nonlinear terms $\mathbf{f}(\mathbf{x}(\tau))$

(b) These bases are then linked by the discrete empirical interpolation method (DEIM).

The formulation for DEIM requires certain assumptions on the structure of nonlinear terms. This will be explored in the rest of this chapter.

\subsubsection{Approximation of the Nonlinear Function}

As an effective way to overcome the difficulty associated with the repetitive evaluation of nonlinear function is to approximate the (5.5) by projecting it onto a reduced subspace having a dimension of $k \ll n$. The DEIM provides a methodology to approximate the nonlinear function by combining the projection and interpolation techniques. To this end, a basis $\mathbf{U} \in \mathbb{R}^{n \times k}$ is required to span the reduced subspace in which the trajectory of nonlinear function can reside as

$$
\mathbf{f}\left(\mathbf{x}\left(\mathbf{t}_{\mathbf{i}}\right)\right) \in \operatorname{colspan}\{\mathbf{U}\}, \quad \text { where } \quad t_{i} \in \boldsymbol{\Lambda}_{t}=\left\{t_{1}, t_{2}, \ldots, t_{N_{f}}\right\}
$$


Following the POD scheme $\mathbf{U}$ is computed by using the snapshots of nonlinear function $\mathbf{f}\left(\mathbf{x}\left(t_{i}\right)\right)$ obtained through simulation for some training input, evaluated at the selected spatial points $\Lambda_{t}$ as

$$
\mathcal{X}_{f}=\left[\mathbf{f}\left(\mathbf{x}\left(t_{i}\right)\right) \mid t_{i} \in \boldsymbol{\Lambda}_{t}\right] \in \mathbb{R}^{n \times N}, \quad \text { where } \quad N \ll n
$$

The samples of

The matrix $\mathbf{Q} \in \mathbb{R}^{n \times m}$ which is the basis for the states $\mathbf{x}\left(t_{i}\right)$ is also obtained based on the POD approach as explained in Section 3.4. The samples of response $\mathbf{x}\left(t_{i}\right)$ are obtained through the simulation mentioned above and the snapshots are also evaluated as

$$
\mathbf{x}\left(\mathbf{t}_{\mathbf{i}}\right) \in \operatorname{colspan}\{\mathbf{Q}\}, \quad \text { where } \quad t_{i} \in \boldsymbol{\Lambda}_{t}
$$

collected in the columns of a data matrix as

$$
\boldsymbol{\mathcal { X }}_{x}=\left[\mathbf{x}\left(t_{i}\right) \mid t_{i} \in \boldsymbol{\Lambda}_{t}\right] \in \mathbb{R}^{n \times N}
$$

Then, the projection matrices are obtained by applying Singular Value Decomposition (SVD) to the two snapshot matrices, as

$$
\begin{aligned}
& \mathcal{X}_{f}=\mathbf{V}_{f} \boldsymbol{\Sigma}_{f} \mathbf{U}_{f}^{t}, \quad \text { where } \quad \mathbf{v}_{f} \in \mathbb{R}^{\mathrm{n} \times \mathrm{N}} \\
& \mathcal{X}_{x}=\mathbf{V}_{x} \boldsymbol{\Sigma}_{x} \mathbf{U}_{x}^{t}, \quad \text { where } \quad \mathbf{v}_{x} \in \mathbb{R}^{\mathrm{n} \times \mathrm{N}}
\end{aligned}
$$

Projection matrix $\mathbf{U}$ is obtained by truncating of $\mathbf{V}_{f}$ to the first $k$ left singular vectors and similarly, $\mathbf{V}_{x}$ is formed using the first $m$ columns of $\mathbf{V}_{f}$ where $k, m<<N<n$. Hence, the reduced variables and the small nonlinear function set will have the original 
interpretations of basic behaviors.

$$
\mathbf{f}(\alpha) \approx \mathbf{U} \mathbf{c}(\alpha)
$$

where $\mathbf{c}(\alpha) \in \mathbb{R}^{k}$ is the corresponding coefficient vector. To simplify the form of equations in establishing the concept, a parameter $\alpha$ is used here. But for the sake of generality, hereafter, $\alpha$ is given to present both time and system parameter. Unless when the differentiation is necessary.

To find $\mathbf{c}(\alpha)$, the DEIM theory introduces a method which is to select the first $k$ distinguished rows from the overdetermined system in (5.12) [3], which requires a selection matrix $\mathbf{P} \in \mathbb{R}^{n \times k}$ as

$$
\mathbf{P}=\left[\mathbf{e}_{\gamma_{1}}, \mathbf{e}_{\gamma_{2}}, \ldots, \mathbf{e}_{\gamma_{i}}, \ldots, \mathbf{e}_{\gamma_{k}}\right]_{n \times k}
$$

where

$$
\mathbf{e}_{\gamma_{i}}=\left[\begin{array}{c}
0 \\
\vdots \\
1 \\
\vdots \\
0
\end{array}\right]_{n \times 1}
$$

where $\mathbf{e}_{\gamma_{i}}$ is the $\gamma_{i}^{\text {th }}$ column of the identity matrix $\mathbf{I}_{n \times n}, \gamma_{i} \in \boldsymbol{\Gamma}$, and

$$
\boldsymbol{\Gamma} \triangleq\left[\gamma_{1}, \gamma_{2}, \cdots, \gamma_{k}\right] \in \mathbb{N}^{k}, \quad \gamma_{i} \in\{1,2, \ldots, N\}
$$

$\gamma_{i}$ is the selection parameter which is found from the DEIM algorithm and collected 
in $\Gamma$ (5.15). Deciding $\Gamma$ leads to defining the selection matrix $\mathbf{P}$ as shown in (5.13) which is to select the significant entries of the nonlinear function as

$$
\mathbf{P}^{t} \mathbf{f}(\alpha)=\left(\mathbf{P}^{t} \mathbf{U}\right) \mathbf{c}(\alpha)
$$

The following is an illustrative approach to exemplify the from of resulting vectors in the both left and right hand sides of (5.16),

L.H.S:

$$
\begin{aligned}
& \mathbf{P}^{t} \mathbf{f}(\alpha)=\left[\begin{array}{c}
\mathbf{e}_{\gamma_{1}}^{t} \\
\mathbf{e}_{\gamma_{2}}^{t} \\
\mathbf{e}_{\gamma_{3}}^{t}
\end{array}\right]_{3 \times n}\left[\begin{array}{c}
f_{1}(\alpha) \\
\vdots \\
f_{i}(\alpha) \\
\vdots \\
f_{n}(\alpha)
\end{array}\right]_{n \times 1}=\left[\begin{array}{c}
f_{\gamma_{1}}(\alpha) \\
f_{\gamma_{2}}(\alpha) \\
f_{\gamma_{3}}(\alpha)
\end{array}\right]
\end{aligned}
$$

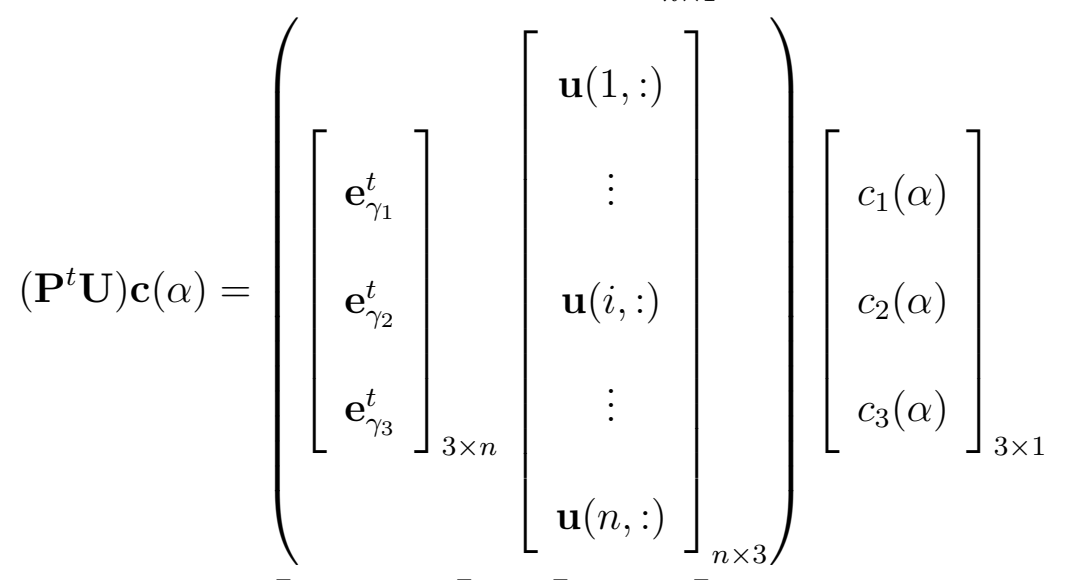

$$
\begin{aligned}
& =\left[\begin{array}{l}
\mathbf{u}_{\text {row\# } \gamma_{1}} \\
\mathbf{u}_{r o w \# \gamma_{2}} \\
\mathbf{u}_{\text {row\# } \gamma_{3}}
\end{array}\right]_{3 \times k}\left[\begin{array}{c}
c_{1}(\alpha) \\
c_{2}(\alpha) \\
c_{3}(\alpha)
\end{array}\right]_{3 \times 1}
\end{aligned}
$$


From (5.17) and (5.18), it is seen that

$$
\left[\begin{array}{c}
f_{\gamma_{1}}(\alpha) \\
f_{\gamma_{2}}(\alpha) \\
f_{\gamma_{3}}(\alpha)
\end{array}\right]=\left[\begin{array}{c}
\mathbf{u}_{\text {row\# } \gamma_{1}} \\
\mathbf{u}_{\text {row\# } \gamma_{2}} \\
\mathbf{u}_{\text {row\# } \gamma_{3}}
\end{array}\right]\left[\begin{array}{l}
c_{1}(\alpha) \\
c_{2}(\alpha) \\
c_{3}(\alpha)
\end{array}\right]
$$

Assuming that the square matrix $\left(\mathbf{P}^{t}\right)_{k \times n} \mathbf{U}_{n \times k}$ is (arguably) invertible (non-singular), from (5.16) the coefficient vector can be found as

$$
\mathbf{c}(\alpha)=\left(\mathbf{P}^{t} \mathbf{U}\right)^{-1} \mathbf{P}^{t} \mathbf{f}(\alpha)
$$

Combined (5.20) with (5.12), an approximation for the nonlinear function is give as

$$
\mathbf{f}(\alpha) \approx \mathbf{U} \mathbf{c}(\alpha)=\mathbf{U}\left(\mathbf{P}^{t} \mathbf{U}\right)^{-1} \mathbf{P}^{t} \mathbf{f}(\alpha)
$$

The accuracy of this approximation depends on the quality of

- The projection matrix for the nonlinear function $\mathbf{U}$ (basis spanning the subspace of $\mathbf{f}(\alpha)$ )

- The interpolation parameter $\Gamma$ which forms the selection matrix $\mathbf{P}$ (selecting nonlinear functions in $\mathbf{f}(\alpha)$ )

Using (5.20), an approximation for the nonlinear function of a dynamic system can be obtained as

$$
\begin{aligned}
\mathbf{f}(\mathbf{x})=\mathbf{f}(\mathbf{Q} \hat{\mathbf{x}}) & \approx \mathbf{U}\left(\mathbf{P}^{t} \mathbf{U}\right)^{-1} \mathbf{P}^{t} \mathbf{f}(\mathbf{Q} \hat{\mathbf{x}}) \\
& =\mathbf{U}\left(\mathbf{P}^{t} \mathbf{U}\right)^{-1} \tilde{\mathbf{f}}\left(\mathbf{P}^{t} \mathbf{Q} \hat{\mathbf{x}}\right)
\end{aligned}
$$


Based on (3.22), an approximation for the reduced nonlinear function is given as

$$
\hat{\mathbf{f}}(\hat{\mathbf{x}})=\underbrace{\mathbf{Q}^{t} \mathbf{U}\left(\mathbf{P}^{t} \mathbf{U}\right)^{-1}}_{m \times k} \underbrace{\tilde{\mathbf{f}}\left(\mathbf{P}^{t} \mathbf{Q} \hat{\mathbf{x}}\right)}_{k \times 1}
$$

It is to be noted that, the followings parts in (5.23) are not dependent on the value of $\alpha$.

$$
\begin{aligned}
& \boldsymbol{\Xi} \triangleq \mathbf{Q}^{t} \mathbf{U}\left(\mathbf{P}^{t} \mathbf{U}\right)^{-1} \in \mathbb{R}^{m \times k} \\
& \boldsymbol{\Theta} \triangleq \mathbf{P}^{t} \mathbf{Q} \in \mathbb{R}^{k \times m}
\end{aligned}
$$

The matrices $\boldsymbol{\Xi}$ (5.24) and $\boldsymbol{\Theta}$ (5.25) need to be precomputed only once before running the Newton-Raphson iterations. Having $m, k \ll n$, these dense matrices are very small and hence very efficient to work with. Specifically, instead of performing the multiplication on the matrices of the original size, only the multiplication in an order of $k$ for each iteration is required. This solves the problem of high CPU-cost needed for the simulation of the reduced nonlinear function for every iteration. Also, instead of repetitively computing the nonlinear function in the original domain, the new approach only needs to build a nonlinear function of order $k$. This is because $\tilde{\mathbf{f}}(\Theta \hat{\mathbf{x}}) \in \mathbb{R}^{k}$ can be obtained through evaluation of $k$ components of the nonlinear function where $k \ll n$. A selection matrix $\Gamma$ in (5.15) is used to extract the $k$ corresponding rows of the nonlinear function. The new approximation makes the evaluation of the nonlinearity and simulating the nonlinear reduced order model independent of the original system's order. In particular, the complexity of computing the following reduced order function

$$
\hat{\mathbf{f}}(\hat{\mathbf{x}})=\boldsymbol{\Xi} \tilde{\mathbf{f}}(\Theta \hat{\mathbf{x}})
$$


includes the cost for evaluating the $k$ nonlinear function $f_{\gamma_{i}}$ with $k$ components is $\mathcal{O}(\alpha(k))$, where $\alpha(\cdot)$ is some function of $k$. Then the complexity for computing (5.26) is approximately $\mathcal{O}(\alpha(k)+4 m k)[3]$.

\subsubsection{Approximation of the Jacobian Function}

The Jacobian matrix should also be reduced into the same order. The same projection

basis with the nonlinear function $\Xi(5.24)$ and $\Theta(5.25)$ are used to approximate the Jacobian (square) matrix. It is

$$
\begin{aligned}
\hat{\mathbf{J}}(\hat{\mathbf{x}}) & =\underbrace{\mathbf{Q}^{t} \mathbf{U}\left(\mathbf{P}^{t} \mathbf{U}\right)^{-1}}_{m \times k} \underbrace{\tilde{\mathbf{J}}\left(\mathbf{P}^{t} \mathbf{Q} \hat{\mathbf{x}}\right)}_{k \times n} \underbrace{\mathbf{P}^{t} \mathbf{Q}}_{k \times m} \\
& =\boldsymbol{\Xi} \tilde{\mathbf{J}}(\boldsymbol{\Theta} \hat{\mathbf{x}}) \boldsymbol{\Theta}
\end{aligned}
$$

where the DEIM approximation for the Jacobian is given as

$$
\begin{aligned}
\tilde{\mathbf{J}}\left(\mathbf{P}^{t} \mathbf{Q} \hat{\mathbf{x}}\right) & =\tilde{\mathbf{J}}(\boldsymbol{\Theta} \hat{\mathbf{x}})=\tilde{\mathbf{J}}\left(y_{\gamma i}^{\prime}\left(x_{\gamma i}\right)\right) \\
& =\operatorname{diag}\left\{\mathbf{f}^{\prime}\left(y_{\gamma 1}\left(x_{\gamma 1}\right)\right), \cdots, \mathbf{f}^{\prime}\left(y_{\gamma k}\left(x_{\gamma k}\right)\right)\right\}
\end{aligned}
$$

This is to (a) select the corresponding nonlinear functions, (b) find their derivative, and (c) put the result back as a diagonal matrix. The computational complexity for the approximation is about $\mathcal{O}\left(\alpha(k)+2 m k+2 \beta m k+2 m k^{2}\right)$, where $\beta$ is the number of nonzero entries in Jacobian matrix.

This format is given under the assumption that Jacobian is a diagonal matrix. This requires certain limitations in the geometry of the circuits. There are that, (a) every nonlinear element is only connected between a node and the ground, and (b) each nonlinear function is only depended on the variable associated with its own node.

The general case will be presented in section 5.4. 


\subsection{Analysis and Algorithm for Interpolation In- dices}

The algorithm to find the selection matrix $\mathbf{P}$ (5.13) and indices $\mathbf{\Gamma}$ (5.15) is presented in Algorithm 3 in this section. In the DEIM process these selection operators $\mathbf{P}$ and $\Gamma$ are formed by considering the importance of nonlinear function $f_{i}(\alpha)$. In its turn, this importance is decided based on the contribution of each nonlinear function to the dimensionality of the nonlinear subspace and hence, its effect in limiting the growth of error.

In Algorithm 3, the DEIM algorithm decides the indices $\gamma_{l}$, for $l=1, \ldots$, based on the orthogonal basis $\mathbf{U}$ spanning the subspace of nonlinear behavior. The process begins with selecting the first interpolation index $\gamma_{1}$, of which every index should be in the range from 1 to $\mathrm{n}$ corresponding with the entries of the nonlinear term. $\gamma_{1}$ also corresponds with the entry of the first basis $\mathbf{u}_{1}$ that has the largest magnitude. The rest of selection parameter $\gamma_{i}$, for $i=\{2, \cdots, k\}$ are selected based on the principle that each selection parameter should relate with the corresponding largest magnitude of the residual $\mathbf{r} \triangleq \mathbf{u}_{l}-\mathbf{U c}$. As referred to preciously, the $\mathbf{r}$ can be regarded as the error or the residual with the sum of the previous difference and the value of the next base. For each step, $\mathbf{P}^{t} \mathbf{U}$ is not singular and the DEIM could iterate to the required selection parameter. This also ensures that a selection parameter will not be repeated.

To prove the $\tilde{\mathbf{f}}(\mathbf{x})$ is an interpolation approximation of the original nonlinear function, the following equation should hold. Thus at the Discrete points, the nonlinear function will have an exact match with the reduced approximate nonlinear function. 


$$
\begin{aligned}
\mathbf{P}^{t} \tilde{\mathbf{f}}(\mathbf{x}) & =\mathbf{P}^{t}\left(\mathbf{U}\left(\mathbf{P}^{t} \mathbf{U}\right)^{-1} \mathbf{P}^{t} \mathbf{f}(\mathbf{x})\right) \\
& \approx \mathbf{P}^{t} \mathbf{f}(\mathbf{x})
\end{aligned}
$$

The following Algorithm 3 presents a detailed description on finding the selection matrix.

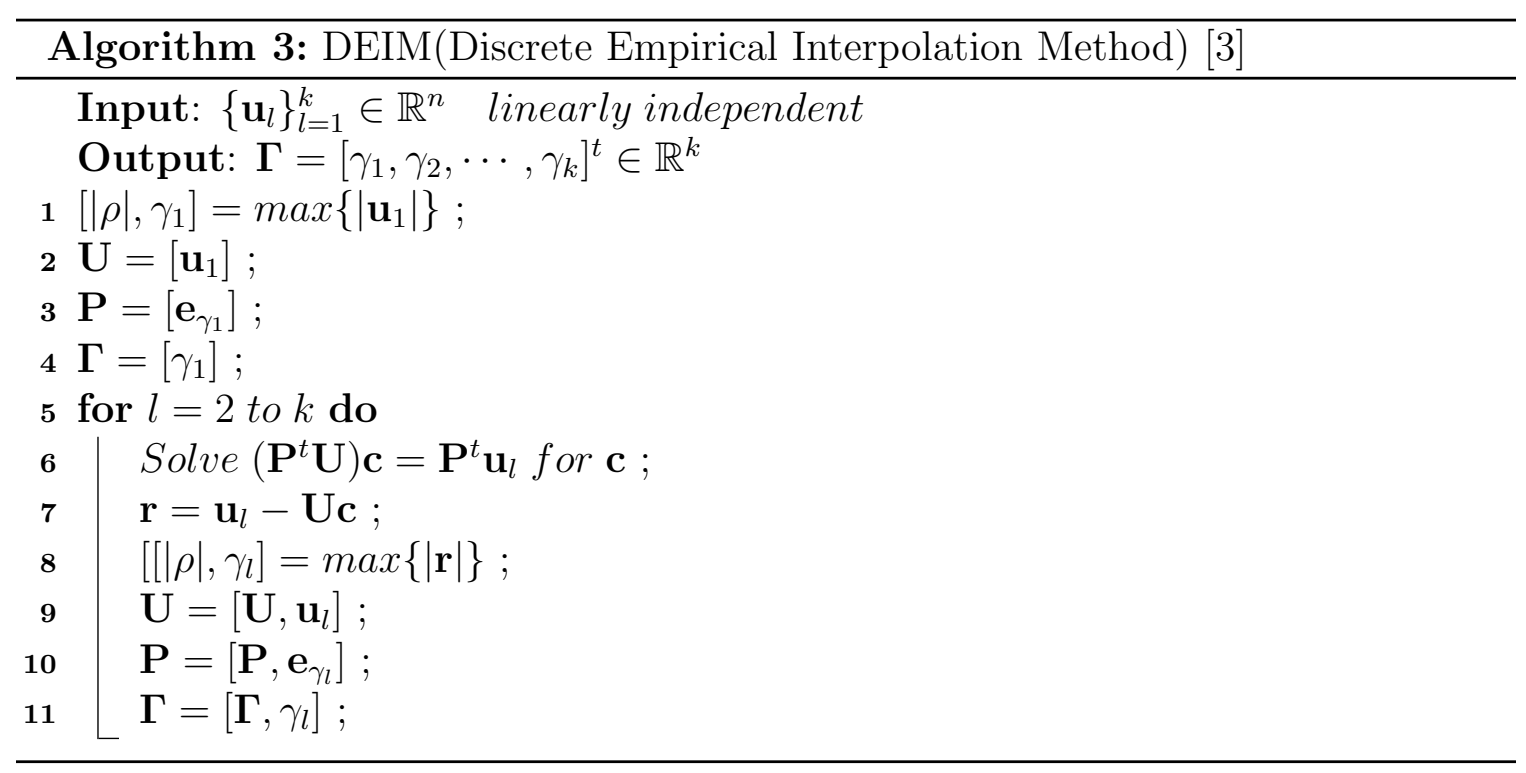

\subsection{Interpolation of General Nonlinear Functions}

As mentioned preciously in the beginning of this chapter, The formulation for DEIM requires certain assumptions on the structure of nonlinear terms. In the previous section, a simple case for nonlinear functions was given to illustrate the algorithm and its underlaying ideas. However, extending DEIM method to the general cases is straightforward. Realistically, $f_{i}(\mathbf{x})$ can not be dependent on all the circuit variables $x_{i}$ for $i=1 \ldots, n$. Thus, being a function of a few selected variables, the $\mathrm{i}^{\text {th }}$ nonlinear 
function in the nonlinear vector $\mathbf{f}(\mathbf{x})$ is given as

$$
f_{i}(\mathbf{x})=f_{i}\left(x_{j_{1}^{i}}, x_{j_{2}^{i}}, \cdots, x_{j_{\sigma}^{i}}\right)=f_{i}\left(\mathbf{x}\left(\mathbf{j}_{i}\right)\right)
$$

The integer vector $\mathbf{j}_{i}$ has a form of:

$$
\mathbf{J}_{i} \triangleq\left[\gamma_{1}^{i}, \gamma_{2}^{i}, \cdots, \gamma_{\sigma}^{i}\right] \in \mathbb{N}^{\sigma}, \quad \gamma_{i} \in\{1,2, \ldots, N\}
$$

It infers a relationship between each nonlinear function with the variables. The parameter $\sigma$ is the total variable numbers which are needed to evaluate the corresponding nonlinear function.

Recall that, (5.23) is for the cases that the Jacobian matrix is diagonal. Hence, in (5.23) the selection operator $\mathbf{P}$ is use for both the nonlinear function and the corresponding variable, i.e.,

$$
\mathbf{P}^{t} \mathbf{f}(\mathbf{Q} \hat{\mathbf{x}})=\tilde{\mathbf{f}}\left(\mathbf{P}^{t} \mathbf{Q} \hat{\mathbf{x}}\right)
$$

Also, $\mathbf{P}^{t} \mathbf{Q}$ can be found by selecting the rows of $\mathbf{Q}$ corresponding to $\Gamma$ through indexing, whereas applying the direct multiplication. However, this is not directly applicable to the general nonlinear cases. For general cases, there is an efficient way to compute the $\mathbf{P}^{t} \mathbf{f}(\mathbf{Q} \hat{\mathbf{x}})$,

$$
\mathbf{P}^{t} \mathbf{f}(\mathbf{Q} \hat{\mathbf{x}})=\mathbf{P}^{t}\left[f_{1}(\mathbf{Q} \hat{\mathbf{x}}), f_{2}(\mathbf{Q} \hat{\mathbf{x}}), \cdots, f_{n}(\mathbf{Q} \hat{\mathbf{x}})\right] \in \mathbb{R}^{n}
$$

Noting that $\mathbf{x}_{j} \approx \mathbf{Q}(j,:) \hat{\mathbf{x}}$, the approximation for each term of the nonlinear function is given as

$$
\tilde{f}_{\gamma_{i}}(\tilde{\mathbf{x}})=\tilde{f}_{\gamma_{i}}\left(\mathbf{Q}\left(j_{\gamma_{i}},:\right) \hat{\mathbf{x}}\right)
$$


Hence, the approximation for $\mathbf{f}(\mathbf{x})$ is considered as

$$
\mathbf{P}^{t} \mathbf{f}(\mathbf{Q} \hat{\mathbf{x}})=\left[f_{\gamma_{1}}\left(\mathbf{Q}\left(j_{\gamma_{1}},:\right) \hat{\mathbf{x}}\right), f_{\gamma_{2}}\left(\mathbf{Q}\left(j_{\gamma_{2}},:\right) \hat{\mathbf{x}}\right), \cdots, f_{\gamma_{m}}\left(\mathbf{Q}\left(j_{\gamma_{m}},:\right) \hat{\mathbf{x}}\right] \in \mathbb{R}^{m}\right.
$$

If $k \ll n$, the computational cost will be low because just a small part of the variables are evaluated for the nonlinear function evaluation. The final form for the general case is

$$
\hat{\mathbf{f}}(\hat{\mathbf{x}}) \approx \mathbf{Q}^{t} \mathbf{U}\left(\mathbf{P}^{t} \mathbf{U}\right)^{-1} \tilde{\mathbf{f}}_{\gamma_{i}}\left(\mathbf{Q}\left(j_{\gamma_{i}},:\right) \hat{\mathbf{x}}\right)
$$

where $i=1 \ldots, k$.

For the Jacobian function, a similar technique can be applied, instead of the form in (5.27), the new form is given as follows

$$
\hat{\mathbf{J}}(\hat{\mathbf{x}}) \approx \mathbf{Q}^{t} \mathbf{U}\left(\mathbf{P}^{t} \mathbf{U}\right)^{-1} \tilde{\mathbf{J}}_{\gamma_{i}}\left(\mathbf{Q}\left(j_{\gamma_{i}},:\right) \hat{\mathbf{x}}\right) \mathbf{Q}
$$

This $\tilde{\mathbf{J}}$ has a size of $k \times n$. For the large scale problems, the Jacobian matrix is always sparse, and the scheme will be very efficient to project the Jacobian matrix into the ROM. Even if the Jacobian is dense, the complexity for reducing the Jacobian will be in $\mathcal{O}(k n)$, where $k$ is the number of the selection or interpolation points.

\subsection{Computational Results and Verification}

In this section, two examples will be presented to verify the POD and POD-DEIM method. The validity of the DEIM idea will provide a solid start for the moment subspace to perform the nonlinear MOR. Due to the DEIM being raised with the POD, it is also necessary to test the DEIM with the result of POD. This test is to find the DC solution of the nonlinear circuit. For the purpose of this thesis, these two examples will be used for testing the existing algorithm and the proposed method. 


\subsubsection{Example I}

The first example is a common emitter BJT amplifier circuit [29]. This circuit is used as a common emitter amplifier with a DC supply of $20 \mathrm{~V}$. The input of the circuit is assumed as $1 \mathrm{~V}$. The transistor is a 3 terminal nonlinear element. It can test the general case nonlinear POD-DEIM. Figure 2.1 shows the topology of the circuit. The BJT in this circuit will be modeled using the Ebers-Moll model [30] and will be shown in figure 5.1:

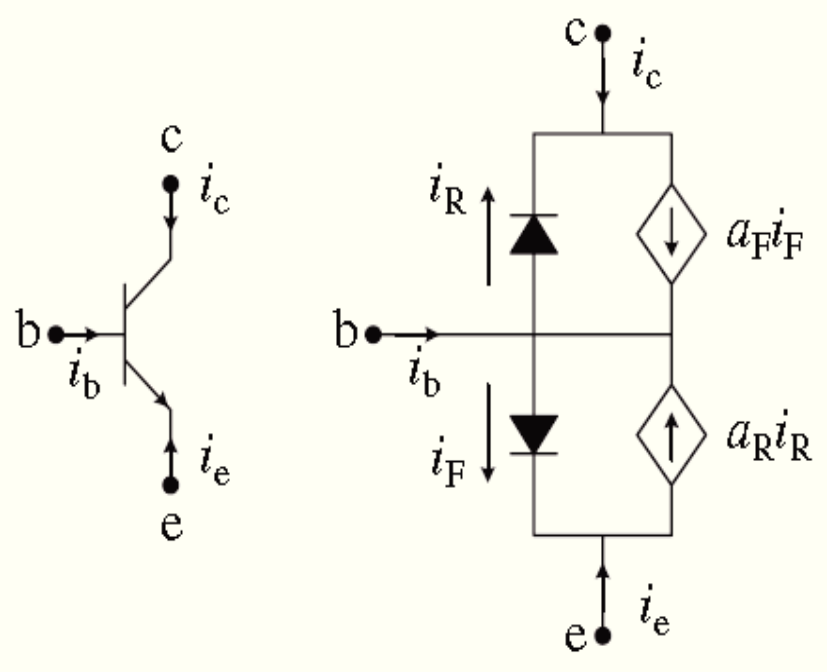

Figure 5.1: Ebers-Moll Model

For this circuit, there are 19 variables, and two tests which will be applied. The first is to only use the POD to reduce the system. In order to solve the reduced system, all variables are needed to be interpolated back to the original domain to form the nonlinear terms to perform the next Newton-Raphson iteration. The second test is to use POD-DEIM for the MOR of the circuit. The main aim of this test is to verify the theory of DEIM for the general nonlinear case.

The total Newton-Raphson iteration number will be tested, and the time to complete the DC solution by using the original source stepping and the corresponding 
method will be compared.

The result of the POD for the BJT circuit is shown as follows:

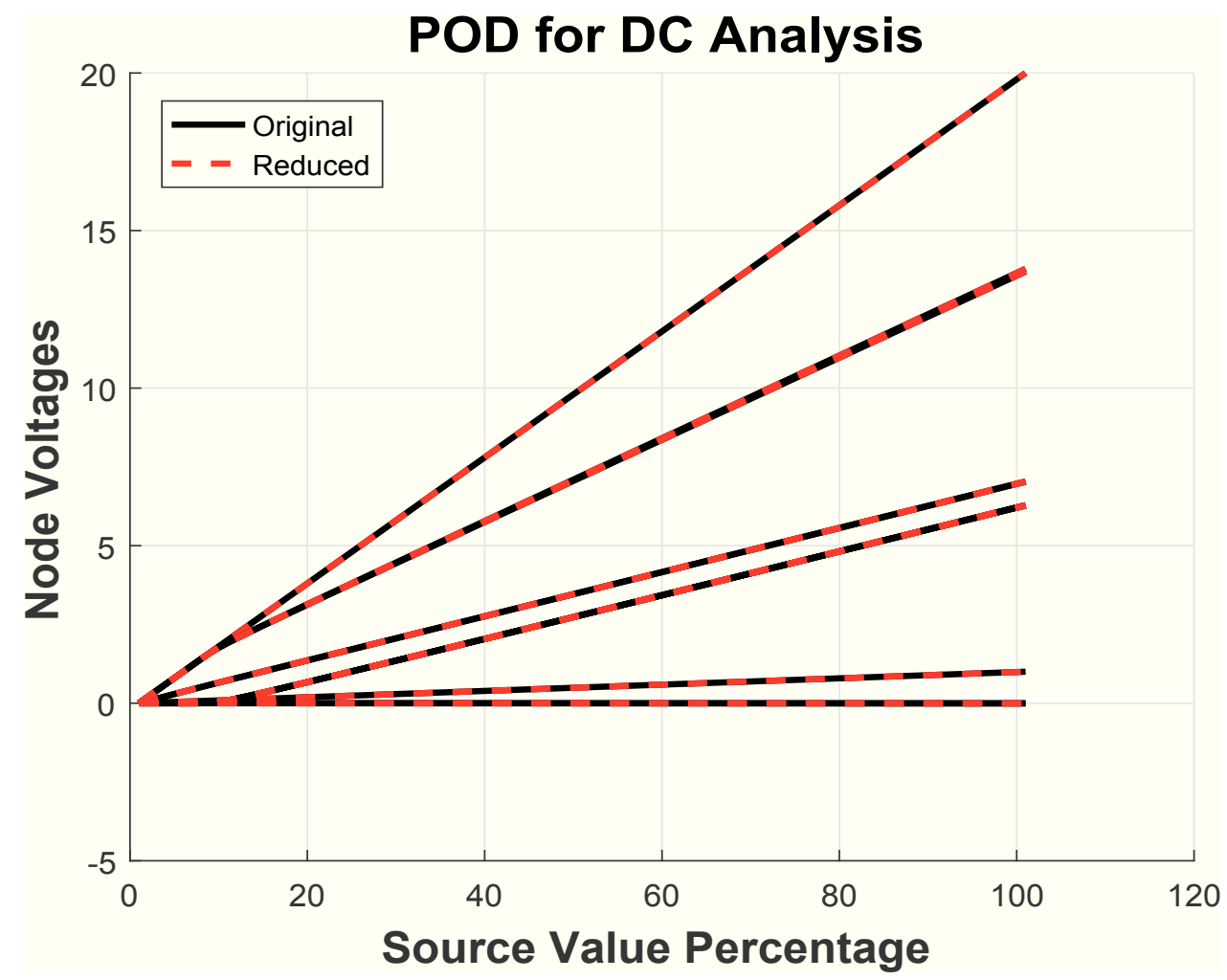

Figure 5.2: Example I: POD DC Analysis

The minimum moment for the POD is 3 , The following table shows the total iterations and speed comparison.

Table 5.1: POD Method for Example I

\begin{tabular}{|c|c|c|c|}
\hline & Model Size & Iteration Number & CPU time (s) \\
\hline Original Method & 19 & 74 & 0.0938 \\
\hline POD Method & 3 & 61 & $\begin{array}{c}\text { Build ROM: } 0.0031 \\
\text { DC Solution: } 0.0328\end{array}$ \\
\hline
\end{tabular}

Rather than using the POD, if the POD-DEIM method is applied to this circuit to find the DC solution, the result would be: 


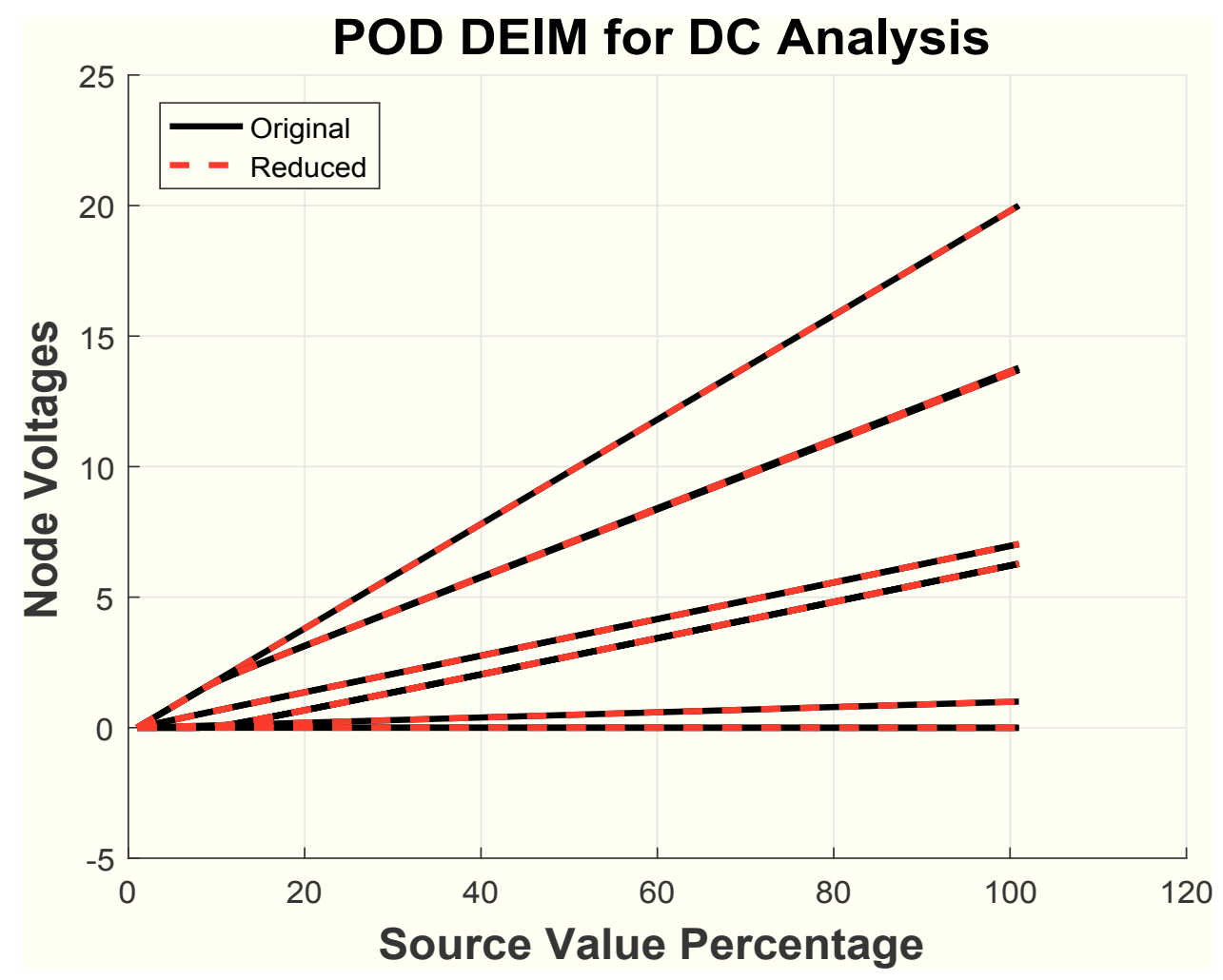

Figure 5.3: Example I: POD-DEIM DC Analysis

The minimum moment requirement number is only 2 , and it also requires order 2 to perform the nonlinear selection for DEIM. The speed and the total iteration number is

Table 5.2: POD-DEIM Method for Example I

\begin{tabular}{|c|c|c|c|}
\hline Method & Model Size & $\begin{array}{c}\text { Iteration } \\
\text { Number }\end{array}$ & CPU time (s) \\
\hline Original & 19 & 74 & 0.0938 \\
\hline POD-DEIM & $\begin{array}{c}\text { Moments: } 2 \\
\text { Nonlinear Selection: } 2\end{array}$ & 73 & $\begin{array}{c}\text { Build ROM: 0.0132 } \\
\text { DC Solution: } 0.0421\end{array}$ \\
\hline
\end{tabular}

By applying the POD-DEIM, the reduced model order will be 2, and it only needs to evaluate 2 nonlinear functions instead of finding all entries of the nonlinear function. There are two reasons why the speed of the POD-DEIM is lower than 
POD. The first consideration is that by performing DEIM, it needs extra time to find the moments of the nonlinear function. The second reason may come to the circuit topology. Specifically, this circuit contains only three nonlinear terms, thus the speed to evaluate the nonlinear function may be faster to conduct the evaluation directly. The LU decomposition speed up would not be very dramatic even though the speed up has a clear expression in Example II. By verifying this BJT circuit, the DEIM theory could be used for the nonlinear circuit MOR to do the DC analysis.

\subsubsection{Example II}

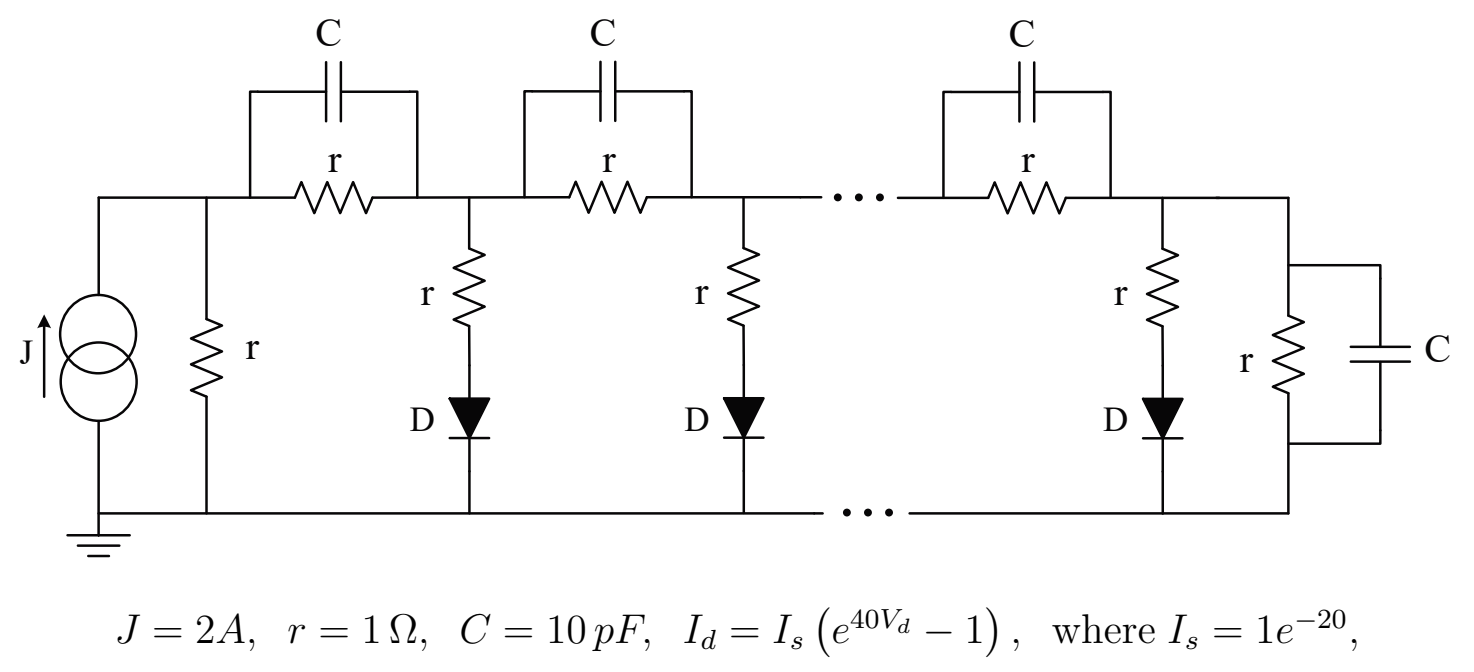

Number of segments $(\mathrm{n})=100$

Figure 5.4: Diode Ladder Circuit (Example II).

Figure 5.4 shows the topology of the diode ladder circuit. This method is to test the special case for the DEIM. This model contains 100 segments. Each segment has two resistors, a capacitor and a diode. The total order of the circuit is 201, and in this circuit, there are 100 nonlinear functions. The LU decomposition speed up can be shown more clearly in this example than the previous one.

The result of the POD method is shown as follows: 


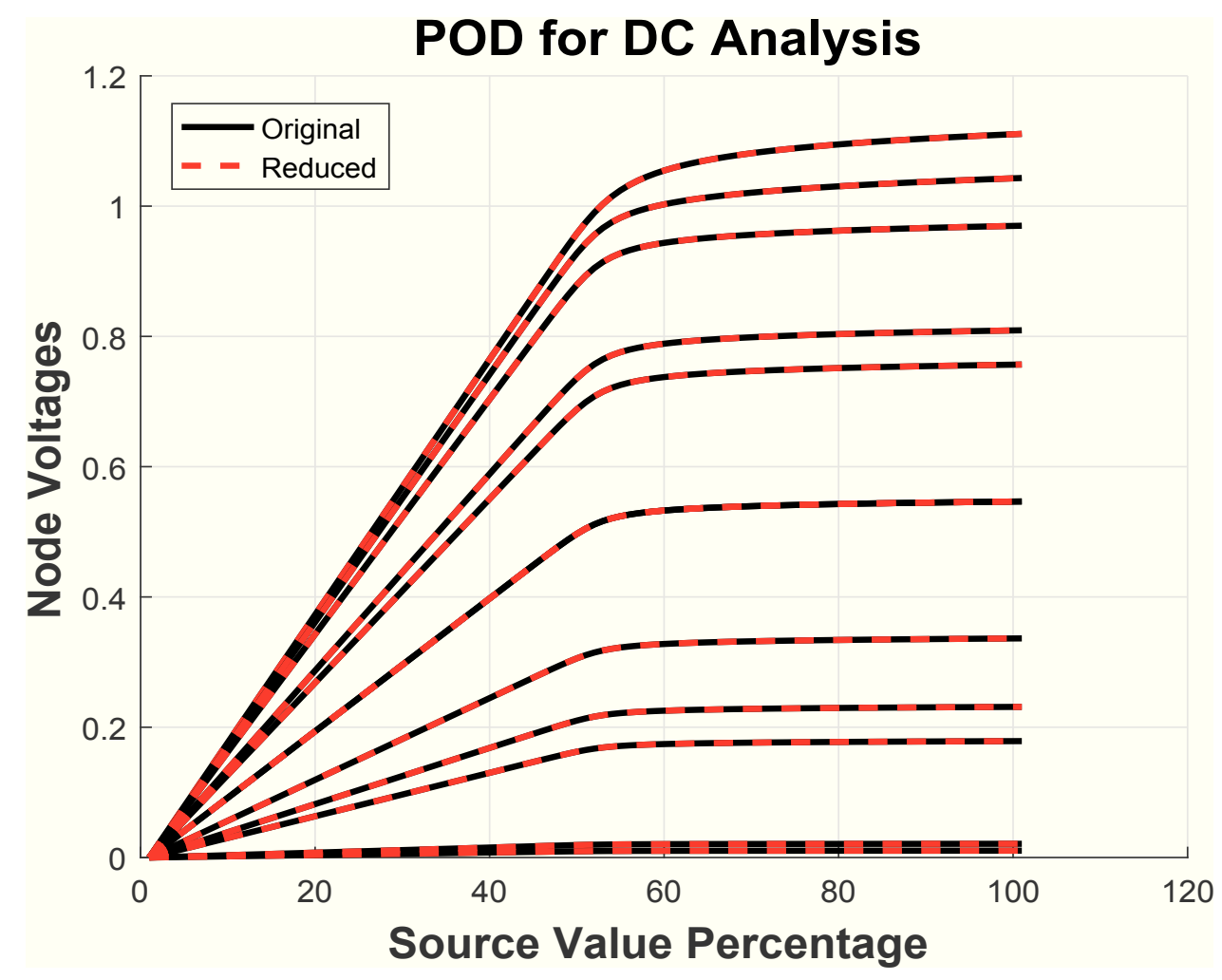

Figure 5.5: Example II: POD DC Analysis

For this example, at least 5 moments for the reduced model are required. The following table shows the simulation result:

Table 5.3: POD Method for Example II

\begin{tabular}{|c|c|c|c|}
\hline & Model Size & Iteration Number & CPU time (s) \\
\hline Original Method & 19 & 613 & 9.0466 \\
\hline POD-DEIM Method & Moments: 5 & 358 & $\begin{array}{c}\text { Build ROM: 0.0564 } \\
\text { DC Solution: 4.4185 }\end{array}$ \\
\hline
\end{tabular}

The result of the POD-DEIM is also obtained and shown as follows: 


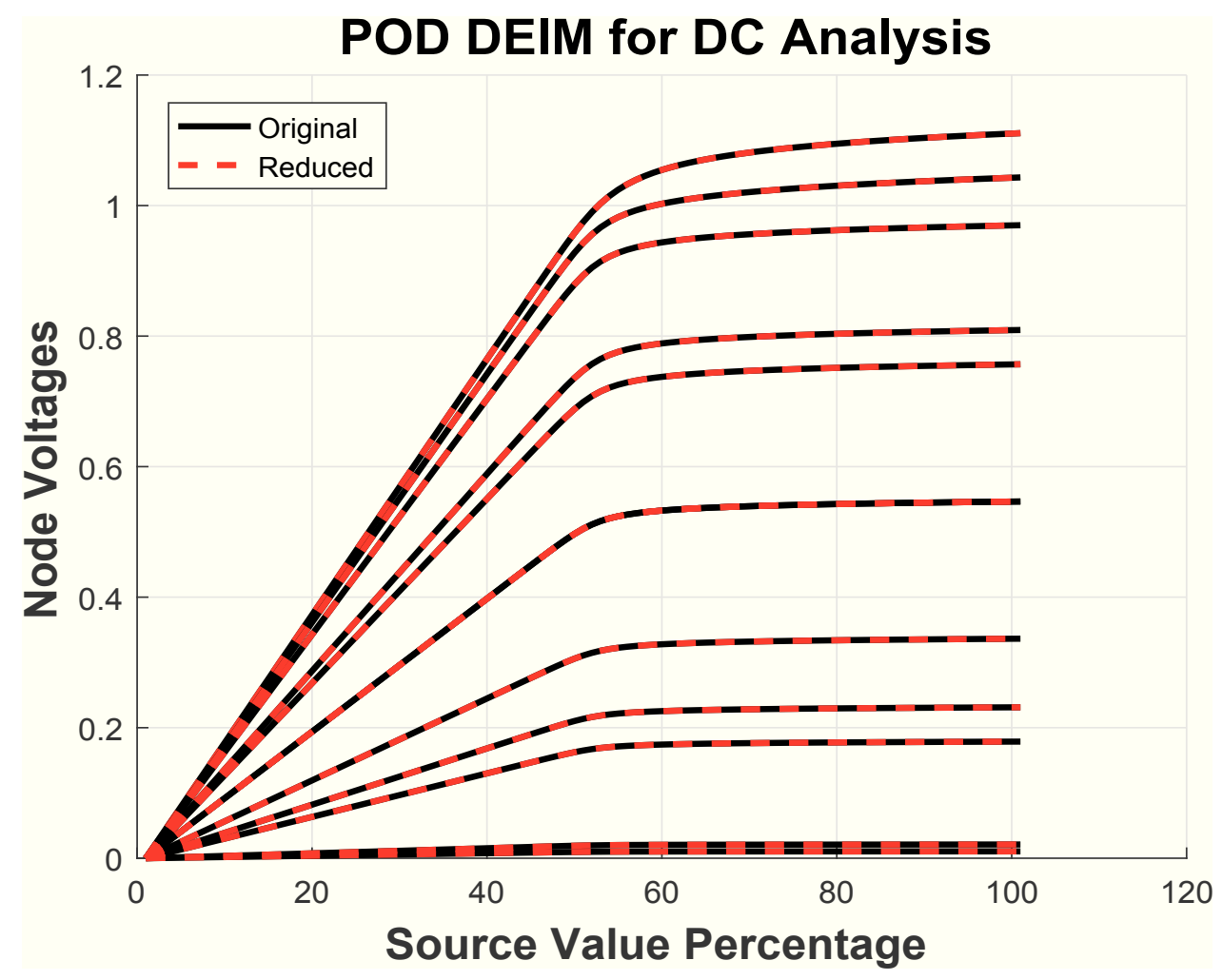

Figure 5.6: Example II: POD-DEIM DC Analysis

If the POD-DEIM method is used, it also needs 5 moments for the reduced model, and it also needs 5 order selection matrix to perform the DEIM. The speed up, iteration number is shown as follows:

Table 5.4: POD-DEIM Method for Example II

\begin{tabular}{|c|c|c|c|}
\hline Method & Model Size & $\begin{array}{c}\text { Iteration } \\
\text { Number }\end{array}$ & CPU time (s) \\
\hline Original & 19 & 613 & 9.0466 \\
\hline POD-DEIM & $\begin{array}{c}\text { Moments: } 5 \\
\text { Nonlinear Selection: } 5\end{array}$ & 468 & $\begin{array}{c}\text { Build ROM: 0.0658 } \\
\text { DC Solution: 0.9477 }\end{array}$ \\
\hline
\end{tabular}

Comparing the result obtained from POD and POD-DEIM, the POD seems to use less time to generate the reduced order model. This is because the POD method does not need to find the moments for the nonlinear function, but the POD method does 
not have a dramatic speed up compared with POD-DEIM. For every LU iteration, all variables should be projected back to the original domain, and 100 nonlinear functions will need to be evaluated. For the POD method, it could only speed up 2 times quicker, but for the POD-DEIM method, the speed up is almost 9 times faster. Thus as the circuit size increases and the total entries of the nonlinear function number increase, the system will have a better performance of the speed up characteristic. It is because the reduced system still needs to apply the MOR for the reduced nonlinear function and the Jacobian matrix. If the system is small, the total time NewtonRaphson saves is covered by the time of the MOR time at each iteration.

The POD test is a good test to verify the theory of the DEIM, but it could not be applied to the DC analysis for the nonlinear system MOR. The prerequisite to use the POD is the snapshots and the nonlinear snapshots, which result from the full circuit test. The circuit is therefore solved before the MOR is done. 


\section{Chapter 6}

\section{Proposed Model Order Reduction for DC}

\section{Analysis}

In chapter 4 , the thesis introduced the DEIM algorithm to solve the problem that the MOR of the Nonlinear system has to evaluate every nonlinear term to perform the Newton-Raphson iteration. The POD technique was used to find the bases and

moments. A new algorithm is needed to find the moments and nonlinear moments without the prerequisite of testing the circuit.

This chapter is organized as follows. The section 6.1 explains the method to find the nonlinear moments. The Section 6.2 provides an illustration of how to use these sorts of moments to find the moment subspace and perform the MOR with DEIM. Next, a computation problem of the new method will be given, and the required modification will be applied in section 6.3. Finally, the simulation result and comparison is given in section 6.4 . 


\subsection{Computation of the Nonlinear Function Mo- ments}

To use the DEIM for the MOR, it is necessary to find the nonlinear moment. The nonlinear moments will be used for finding the selection matrix and the nonlinear bases for the DEIM algorithm.

In Section 4.1.2, the thesis shows the proof of the moments computation format, and a recursive approach to find the moments is outlined. This section provides an example to illustrate the mathematical format for the higher-order moments of the nonlinear function. An exponential characteristic function of a diode is used as an example.

\subsubsection{Computation of the Jacobian Function Moments}

From section 4.1.2, the iteration format to find the moments is outlined, however, to compute the moments, it still needs to find the Jacobian Function Moments. The nonlinear function for the diode is

$$
f(v)=I_{s}\left(e^{\left(v / V_{T}\right)}-1\right)
$$

The Jacobian of nonlinearity is given in below as the first order derivative of the function with respect to the variable $v$

$$
\zeta(\alpha)=\frac{I_{s}}{V_{T}} e^{\left(v / V_{T}\right)}=J\left(\sum_{i=0} M_{i} \alpha^{i}\right)=\left.\sum_{i=0} \zeta^{(i)}(\alpha)\right|_{\alpha=0} \alpha^{i}
$$


Based on the function (6.2), the Taylor series expansions at $\alpha=0$ are

$$
\begin{aligned}
x & =\sum_{i=0} M_{r} \alpha^{i} \\
J(x) & =\sum_{i=0} J_{r} \alpha^{i}
\end{aligned}
$$

where $M_{i}$ represents the scalar moment for the variable, and $J_{i}$ is the scalar moment for Jacobian function. Using (6.2), we get:

$$
\sum_{i=0} J_{i} \alpha^{i}=\frac{I_{s}}{V_{T}} e^{\left(\sum_{i=0} M_{i} \alpha^{i} / V_{T}\right)}
$$

For $\alpha=0$,

$$
\zeta(0)=J\left(M_{0}\right)=\frac{I_{s}}{V_{T}} e^{M_{0} / V_{T}}
$$

and by considering $M_{0}=0$, it is

$$
\zeta(0)=J(0)=\frac{I_{s}}{V_{T}}
$$

From (6.5), the basis equation to find the high order moments for the Jacobian function can be found by taking the first order derivative of it:

$$
\sum_{i=0} i J_{i} \alpha^{i-1}=\frac{I_{s}}{V_{T}^{2}} e^{\left(\sum_{i=0} M_{i} \alpha^{i} / V_{T}\right)} \sum_{i=0} i M_{i} \alpha^{i-1}
$$

A recursive expression for $(m-1)^{\text {th }}$ derivative of the (6.8) with respect to the sweep parameter can be shown as [31]

$$
\zeta^{(i)}=\frac{1}{V_{T}} \sum_{r=0}^{i-1} \zeta^{(r)} M_{i-r}(i-r)
$$




\subsubsection{Computation of the Nonlinear Function Moments}

Based on the exponential function (6.1), the Taylor series expansions at $\alpha=0$ are

$$
\begin{aligned}
v & =\sum_{i=0} M_{r} \alpha^{i} \\
f(v) & =\sum_{i=0} F_{r} \alpha^{i}
\end{aligned}
$$

where $M_{i}$ represents the scalar moment for the variable, and $F_{i}$ is the scalar moment for nonlinear function. Using (6.10) and (6.11) from (6.1) we get

$$
\sum_{i=0} F_{i} \alpha^{i}=I_{s}\left(e^{\left(\sum_{i=0} M_{i} \alpha^{i} / V_{T}\right)}-1\right)
$$

The zero-th moment for $i=0$ is

$$
F_{0}=f(v(\alpha=0))=f\left(M_{0}\right)=I_{s}\left(e^{M_{0} / V_{T}}-1\right)
$$

where knowing that, for $\alpha=0$ by dismissing the effect of DC source in the MNA equation there only can be

$$
M_{0}=v(\alpha=0)=0
$$

and the diode will draw no current, then

$$
F_{0}=f(\alpha=0)=f\left(M_{0}\right)=0
$$

From the format of (6.12), similar with Jacobian function, the basis equation to find the high order moments for the nonlinear function can be found by taking the first 
order derivative of it:

$$
\sum_{i=0} i F_{i} \alpha^{i-1}=\frac{I_{s}}{V_{T}} e^{\left(\sum_{i=0} M_{i} \alpha^{i} / V_{T}\right)} \sum_{i=1} i M_{i} \alpha^{i-1}
$$

With this basis equation, taking the (i-1)th order derivative, it can be used for finding the $i$ th nonlinear moment. Leibniz Theorem will be applied.

From (6.16) it is concluded that

$$
F_{i}=\frac{1}{i V_{T}} \sum_{r=0}^{i-1}(i-r) M_{i-r} F_{r}+\frac{I_{s}}{V_{T}} M_{i}
$$

All moments of the nonlinear functions can be found based on the equation (6.17), then the result will be formed as a nonlinear moment with the size of $n \times 1$.

There is another method to calculate the nonlinear function moments. Nonlinear function moments could be found from moments directly. From the circuit equation:

$$
\begin{aligned}
& \mathbf{F}_{0}=\mathbf{f}\left(\mathbf{x}_{0}\right) \\
& \mathbf{F}_{1}=\mathbf{b}-\mathbf{G M}_{1} \\
& \mathbf{F}_{i}=-\mathbf{G M}_{i} \text { for } i>1
\end{aligned}
$$

If the nonlinear moment of the first method is found correctly, the result is almost the same with the method to find it in the circuit equation.

The relationship between the moments, Jacobian moments and the nonlinear moments could be summarized. The current order moment could be used to find the current order Jacobian moment and the current order nonlinear moment. To find the next order moment, the current order Jacobian moment and the current order moment are needed. 


\subsection{Nonlinear Model Order Reduction with DEIM}

As it introduced in the previous chapter, the moment subspace is not enough to conduct the efficient MOR for nonlinear circuits. If the nonlinear function has to be evaluated fully for every Newton-Raphson iteration, the computational cost will be significant. Thus the nonlinear moments could be used to find the selection matrix and the nonlinear moment subspace.

The relationship of the proposed method and the POD-DEIM method is that the proposed method will find the moments and the nonlinear moments instead of the snapshots and the nonlinear snapshots. Thus the circuit testing is avoided. For the moments, do the QR decomposition to find the projection matrix. The nonlinear moments could be used for finding the nonlinear function basis and the selection matrix. By applying this method, the MOR could be performed with the DEIM method to obtain the reduced model.

The other term which should be brought to attention to is that the order of the nonlinear function moments which may not be the same with the order of the moments. In the figure above, $m$ represents the moment order, and $k$ represents the nonlinear moment order. The moment order $\mathrm{m}$ will have an effect on the reduced model size. In every Newton-Raphson iteration for the reduced model, it will do the operation for the $m \times m$ matrices. The reduced variable also has a length of $m$.

The $k$ represents the total number of the nonlinear function for the reduced model to evaluate. For every Newton-Raphson iteration, only a small number of the variable is needed to be evaluated from the approximated nonlinear function. It can save time to perform the MOR for the nonlinear function and the Jacobian matrix for every Newton-Raphson iteration.

Both $m$ and $k$ should be much smaller than $n$, which is the original order of the 
circuit model. The total moment number needed for the proposed method is the larger one of $m$ and $k$. Both of these parameters controls the accuracy of the ROM and the DC analysis result. 


\subsection{Modification of the Reduced Model}

For the POD method, the circuit testing process has collected all accurate information of the variable and nonlinear through the source multiplier from 0 to 1 . Thus the subspace it expands is a very accurate description of the original space. This accurate subspace allows the ROM to use a small number of moments to complete the projection without a large error.

However, for the moment subspace method, no information is available on the variables' value except when $\alpha=0$. Thus the subspace it expands should locate at $\alpha=0$. It is acceptable for linear circuits or circuits to have little nonlinearity. However, for circuits have many nonlinear equations or have large nonlinearity, more moments and nonlinear moments will be used to conduct the projection. Results will be accurate at the range of the expansion point. If the $\alpha$ is expanded at 0 , results will be evident in the range close to 0 . But the result for $\mathrm{DC}$ analysis corresponding with $\alpha=1$. Thus in order to get a good final result for DC analysis, more moments may be needed than POD for the subspace.

If the requirement for the moment order is high, then the speed up of the proposed method may not be apparent. It cannot reach the initial intention to perform the MOR. Thus the modification should be made for the proposed method if the circuit cannot be reduced into an ideal order. The basic idea is to find moments at $\alpha=0$, and then perform the MOR. With the reduced model, the DC analysis can be started. When the circuit simulation has an error which is bigger than the acceptable value, it will stop the iteration for the source stepping. Only then will the temporary midterm variable be obtained.

This temporary midterm variable is the value which is the result found from the current reduced variable. Because the $\mathbf{Q}$ projection matrix is orthogonal, then the 
relationship to find the temporary midterm variable is:

$$
\mathbf{x}_{m i d}=\mathbf{Q}^{-1} \hat{\mathbf{x}}_{m i d}=\mathbf{Q}^{t} \hat{\mathbf{x}}_{m i d}
$$

It is necessary to repeat the moment subspace expansion again. The end of the previous source step is the beginning of the next source step. For the new source step, it will continue to the end, or to the beginning of the next modification point. The temporary midterm variable will be used as the initial condition to find the moments, nonlinear moments and Jacobian moments.

$$
\begin{aligned}
& \mathbf{M}_{0}=\mathbf{x}_{\text {mid }} \\
& \mathbf{f}_{0}=\mathbf{f}\left(\mathbf{x}_{\text {mid }}\right) \\
& \mathbf{J}_{0}=\mathbf{J}\left(\mathbf{x}_{\text {mid }}\right)
\end{aligned}
$$

The new moment subspace will have a relatively better approximation for the range close to this modification point. Thus a lesser moment number is required than the situation without the modification required to get an accurate approximation. The simulation result of conducting the modification and the situation without the modification will be shown clearly in the next section. 


\subsection{Computational Results and Verification}

Two examples will be used to test the theory of applying the moment subspace method for the MOR. Of importance is whether moments from the moment subspace are suitable for the DEIM algorithm. The modification will be used for the further reduction of the moment limitation.

\subsubsection{Example I}

Example I is the BJT circuit which has 19 variables. Testing parameters are the same as those referenced in the last chapter. The result of the moment subspace method is:

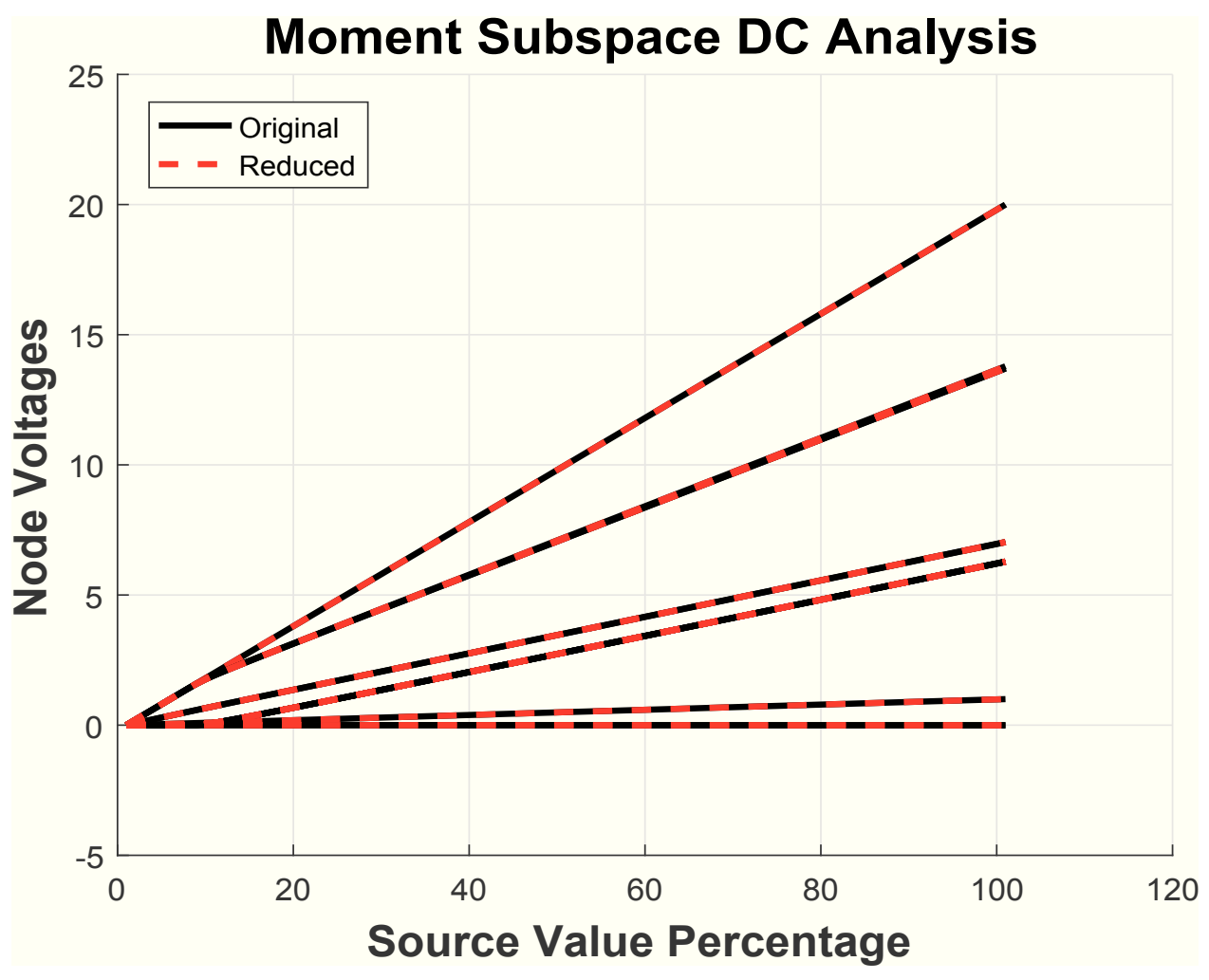

Figure 6.1: Example I: Moment Subspace for DC Analysis 
The minimum moment requirement number is only 4 . The speed up, total iteration number is shown as:

Table 6.1: Moment Subspace Method for Example I

\begin{tabular}{|c|c|c|c|}
\hline & Model Size & Iteration Number & CPU time (s) \\
\hline Original Method & 19 & 74 & 0.0938 \\
\hline $\begin{array}{c}\text { Moment Subspace } \\
\text { Method }\end{array}$ & Moments: & 61 & $\begin{array}{c}\text { Build ROM: } 0.0025 \\
\text { DC Solution: } 0.0334\end{array}$ \\
\hline
\end{tabular}

Adding the DEIM onto the moment subspace method, the result is shown below:

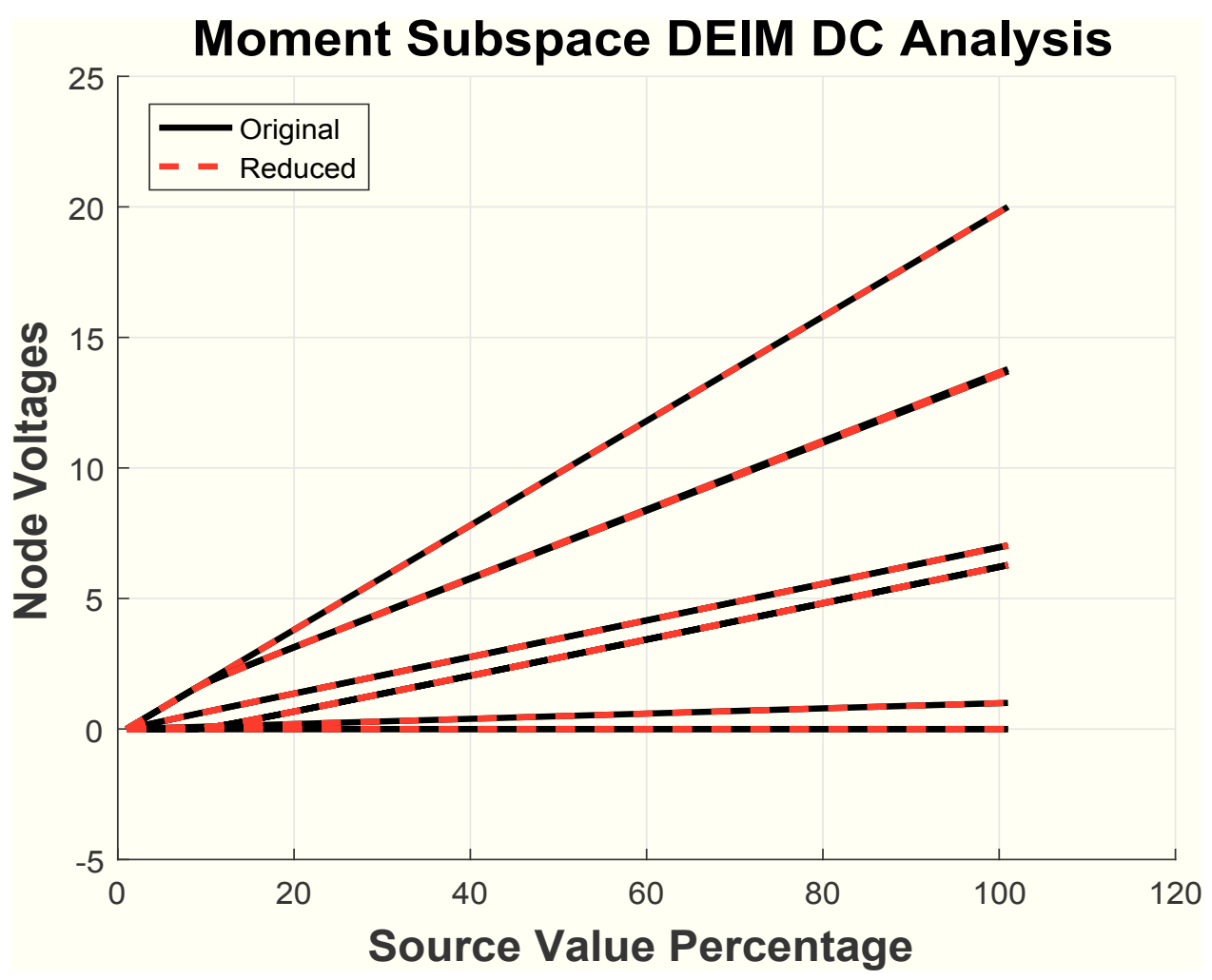

Figure 6.2: Example I: Moment Subspace DEIM for DC Analysis

If DEIM algorithm is added, it also needs 4 moments, but it only needs 3 selections for the nonlinear function, thus only three entries of 19 entries of the nonlinear function vector will be evaluated. The reduced order, speed up, and the total iteration number is: 
Table 6.2: Moment Subspace DEIM Method for Example I

\begin{tabular}{|c|c|c|c|}
\hline & Model Size & Iteration Number & CPU time (s) \\
\hline Original Method & 19 & 74 & 0.0938 \\
\hline $\begin{array}{c}\text { Moment Subspace } \\
\text { Method }\end{array}$ & Moments: 4 & 73 & Build ROM: 0.0171 \\
Nonlinear Moments: 3 & & DC Solution: 0.0334 \\
\hline
\end{tabular}

Similar with the POD method, the reason why the speed up of the moment subspace DEIM is smaller than the moment subspace method is that the computation of computing the nonlinear moments and the Jacobian moments will require time. For each iteration, it has to form the nonlinear function and the Jacobian matrix. However, the biggest contribution for this example is that it verifies the moment subspace DEIM algorithm is correct. Moments and nonlinear moments could totally replace the snapshots and nonlinear snapshots.

The circuit for Example I is smaller than Example II. From the DC source ramping shape, the nonlinearity of three nodes, which connects with three ports of the BJT, is not very dramatic. Additionally, it is not too dense for the nonlinear function vector. Thus the modification is not needed for the simulation of this circuit.

\subsubsection{Example II}

Circuit for Example II has 201 nodes, and there are 100 entries of nonlinear functions. The moment subspace method needed to be tested, and the first step is to test the circuit with 15 moments. The result is shown in figure 6.3. The total number of moments needs to be increased to 20 at a minimum. 


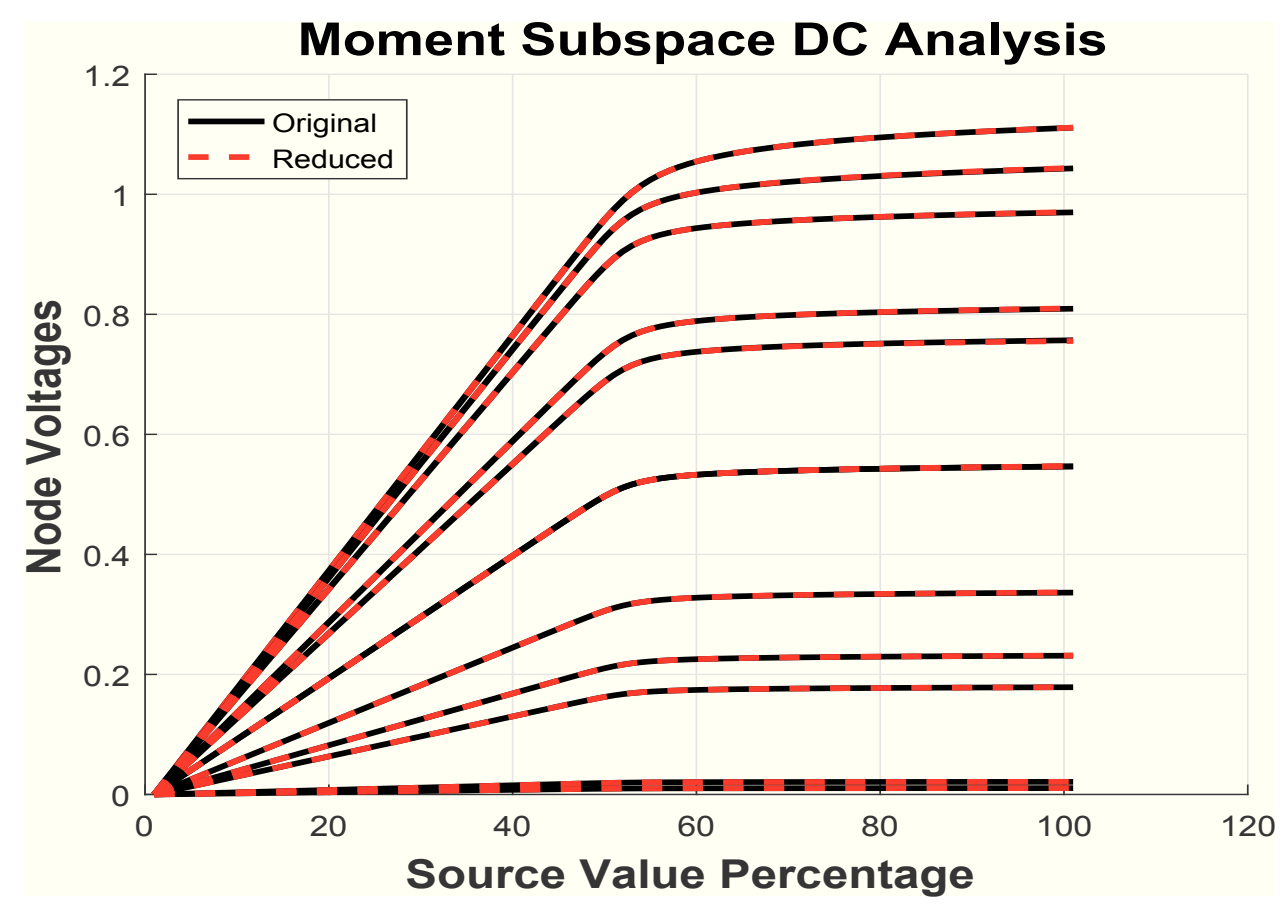

Figure 6.3: Example II: Moment Subspace with 20 Moments for DC Analysis

With 20 moments, the simulation result of the iterations and speed can be measured, and the result is:

Table 6.3: Moment Subspace Method for Example II

\begin{tabular}{|c|c|c|c|}
\hline & Model Size & Iteration Number & CPU time (s) \\
\hline Original Method & 201 & 613 & 9.1264 \\
\hline $\begin{array}{c}\text { Moment Subspace } \\
\text { Method }\end{array}$ & Moments: 20 & 520 & $\begin{array}{c}\text { Build ROM: 0.0746 } \\
\text { DC Solution: } 7.5895\end{array}$ \\
\hline
\end{tabular}

The result shows that by using the moment subspace alone, the speed up is not dramatic. This is because for every Newton-Raphson iteration, the reduced $\hat{\mathbf{x}}$ must be transferred back to the original domain. All nonlinear functions will be evaluated to build new nonlinear function vector and the Jacobian matrix to solve the next Newton-Raphson iteration.

Figure 6.4 shows the result of the proposed moment subspace DEIM method 
result:

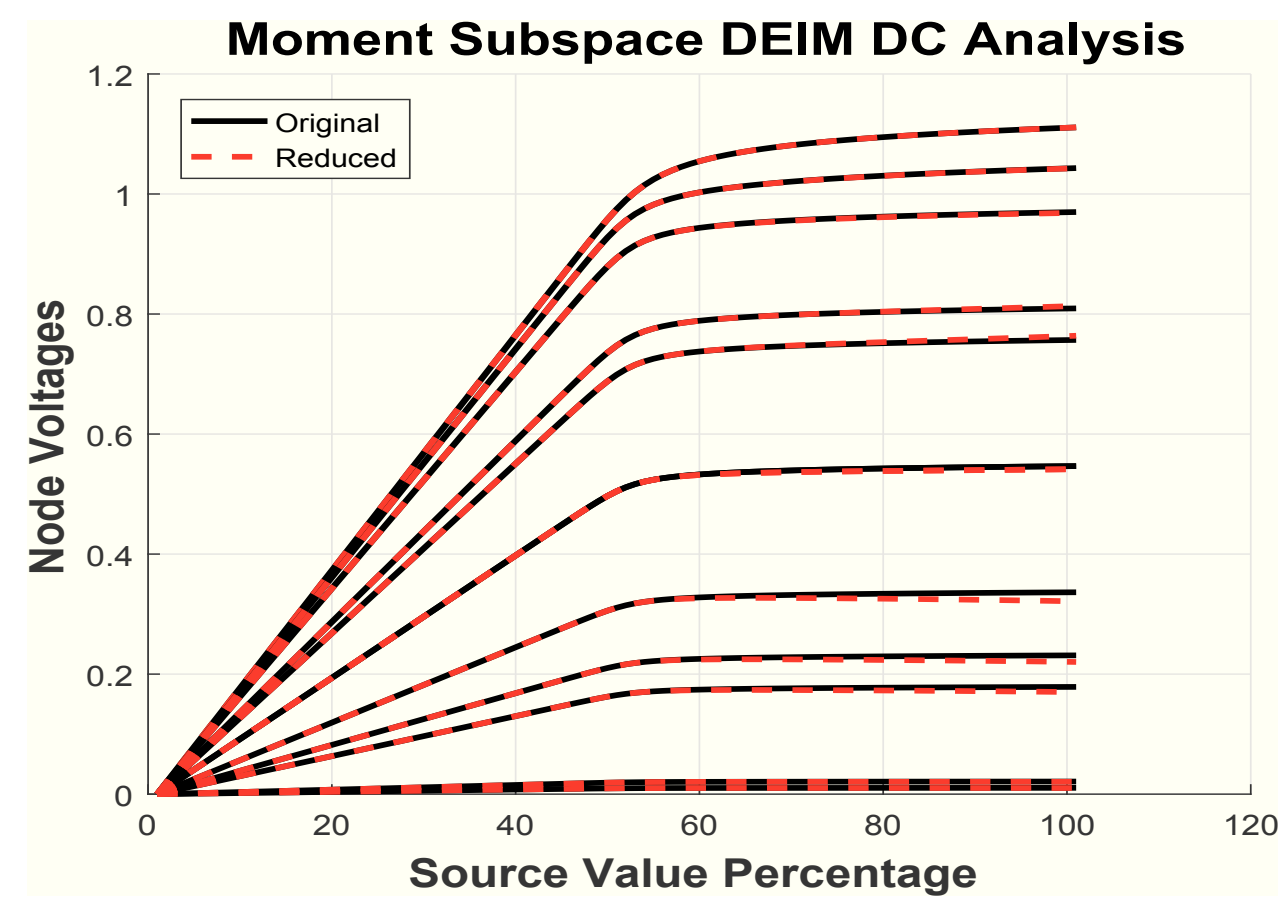

Figure 6.4: Example II: Moment Subspace DEIM with 25 Moments 25 Selections for DC Analysis

Results with an acceptable error could be obtained if 25 moments and 25 order selection matrix is used. The speed and the iteration number is shown in table 6.4:

Table 6.4: Moment Subspace DEIM Method for Example II

\begin{tabular}{|c|c|c|c|}
\hline & Model Size & Iteration Number & CPU time (s) \\
\hline Original Method & 201 & 613 & 9.1264 \\
\hline $\begin{array}{c}\text { Moment Subspace } \\
\text { DEIM Method }\end{array}$ & Moments: 25 & 516 & Build ROM: 0.1925 \\
\end{tabular}

Compared with moment subspace method, the speed up is increased to 3 times approximately. But it still needs to find 25th order moment and 25th nonlinear moment. If less moments are used, the time will be saved because less functions are needed to be evaluated and smaller ROM is used for the Newton-Raphson iteration. Modification will be made at the point $\alpha=0.5$. The result is: 


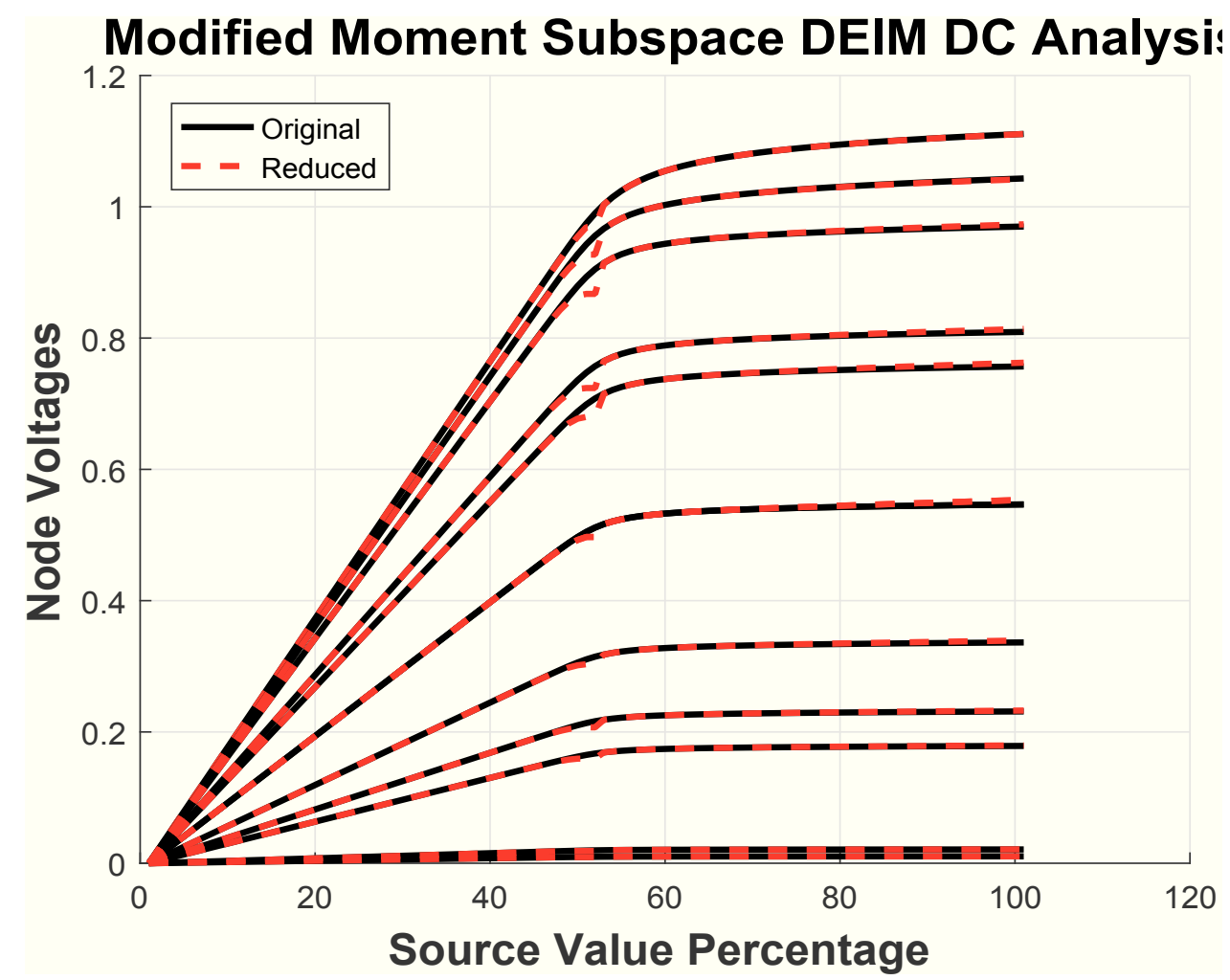

Figure 6.5: Example II: Modified Moment Subspace DEIM with 5 Moments 5 Selections

And the speed, iteration information is shown in table 6.5:

Table 6.5: Modified Moment Subspace DEIM Method for Example II

\begin{tabular}{|c|c|c|c|}
\hline & Model Size & $\begin{array}{c}\text { Iteration } \\
\text { Number }\end{array}$ & CPU time (s) \\
\hline Original Method & 201 & 613 & 9.1264 \\
\hline $\begin{array}{c}\text { Moment Subspace } \\
\text { DEIM Method } \\
\text { (Modified) }\end{array}$ & $\begin{array}{c}\text { Moments 1st: 5 } \\
\text { Monlinear Selection 1st: } 5\end{array}$ & 202 & $\begin{array}{c}\text { Build ROM 1st: 0.0918 } \\
\text { DC Solution 1st: } 0.3701 \\
\text { Build ROM 2nd: } 0.0809 \\
\text { DC Solution 2nd: } 0.4511\end{array}$ \\
\hline
\end{tabular}

For the first part from $\alpha=0$ to 0.5 , it needs at least 5 moments and 5 nonlinear moments. For the second part from $\alpha=0.5$ to 1 , it also needs 5 moments and 5 nonlinear moments. Compared with the result of the moment subspace method, the 
speed up factor is dramatically increased. It will have a 9.2 times speed up over the original source stepping method. Total Newton-Raphson Iteration number is 467, and all are done in the reduced model with order 5 .

From figure 6.5, at the point of $\alpha=0.5$, the curve has an obvious distortion, thus the reduced variables can be projected back to the original domain. The new moments with order 5 can therefore support the new reduced model to reach a good result to the end of $\alpha$.

For this circuit, it has a speed up which is close to 10 times, but for nonlinear circuits which have a larger order, or the ones which are filled with nonlinear function entries, the speed up factor could be larger. Thus this moment subspace DEIM method could be a good algorithm for the complex nonlinear circuit MOR to solve the DC solution. 


\section{Chapter 7}

\section{Parametrized Model Order Reduction}

In the previous chapter, the proposed moment subspace DEIM method is applied for the DC analysis. For which, the source stepping multiplier is the parameter for the Taylor Series expansion. There is a possibility that for any parameters appear outside of the nonlinear function, the moment subspace DEIM could be used to solve the nonlinear equation.

The parameter could appear in the $\mathbf{G}$ matrix, which contains the MNA stamps for memoryless circuit elements. This new circuit formation could be solved with a different value of the parameter.

This chapter will be separated into three sections. The first section is an introduction about the circuit formulation. The second section contains the preferred format of the nonlinear equation to conduct the parameterized analysis based on the DC solution for nonlinear circuits. The third section introduces the method on how to find the moments and nonlinear moments for the new equation format,and presents the result of the simulation. 


\subsection{Parameterized Analysis for Nonlinear DC So- lution}

As was introduced in the previous chapter, the DC Analysis is used to solve initial condition for the nonlinear circuit. The source stepping method has the operation to the source vector. This will raise the question as to whether it is necessary to perform

the DC Analysis again if the electrical element of the nonlinear circuit changes. The parameterized analysis requires the existing DC solution of the original circuit.

Contrary to the equation (2.2), the parameter will not appear on the source vector, it will control the value of the element and appear in the $\mathbf{G}$ matrix:

$$
\begin{aligned}
& \mathbf{G}(\alpha) \mathbf{x}+\mathbf{F}(\mathbf{x})=\mathbf{b} \\
& \mathbf{b}=\mathbf{B u} \\
& \mathbf{y}=\mathbf{L} \mathbf{x}
\end{aligned}
$$

Equations for solving the Newton-Raphson iteration are:

$$
\begin{aligned}
& \mathbf{\Phi}(\mathbf{x})=\mathbf{G}(\alpha) \mathbf{x}+\mathbf{F}(\mathbf{x})-\mathbf{b} \\
& \boldsymbol{\Psi}(\mathbf{x})=\mathbf{G}(\alpha)+\mathbf{J}(\mathbf{x})
\end{aligned}
$$

If the parameter of the circuit changes, it might not necessary to solve the DC solution again. However, with the existing DC solution, new results can be found from the Newton-Raphson iteration. 


\subsection{Reduced Model Formation for DC parameter- ized Analysis}

As introduced above, if the parameter controls the value of the $\mathbf{G}$ instead of the source, the equation will be:

$$
\mathbf{G}(\alpha) \mathbf{x}(\alpha)+\mathbf{f}(\mathbf{x}(\alpha))=\mathbf{b}
$$

For the ease of finding the moments corresponding to the parameter, the circuit formation will be adjusted to the proposed format:

$$
\begin{aligned}
& \mathbf{G}\left(g_{k}+\alpha \times g_{k}\right) \mathbf{x}(\alpha)+\mathbf{f}(\mathbf{x}(\alpha))=\mathbf{b} \\
& \mathbf{\Phi}(\mathbf{x})=\mathbf{G}\left(g_{k}+\alpha \times g_{k}\right) \mathbf{x}(\alpha)+\mathbf{f}(\mathbf{x}(\alpha))-\mathbf{b}=0
\end{aligned}
$$

where $\mathbf{G}\left(g_{k}\right)$ represents the original value of $\mathbf{G}$ matrix, and k relates to the corresponding element. If the resistor connects with a node and ground, then $g_{k}$ has only one entry for the $\mathbf{G}$ matrix. If the resistor connects between two nodes, then $g_{k}$ will have four entries, and $\alpha$ will be multiplied with all these four entries.

When $\alpha=0$, the circuit equation will be the original nonlinear circuit. The result of the function is the $\mathrm{DC}$ solution, and when alpha changes from 0 to 1 , the corresponding entries of the parameter in the matrix $\mathrm{G}$ will become into two times of its original value.

The parameter could also be one time less, and if so, the corresponding entries of the $\mathbf{G}$ matrix would be 0 . The merit of this circuit formation method will be shown in the next section to find moments. 


\subsection{Computation of Derivative and Moments}

The parameterized analysis for the nonlinear circuit has been discussed at length. Chapter 4 described many methods which provided ways to find the projection matrix by using the circuit testing POD method $[32,33]$. There may be a different method to find the moments which is suitable to determine the parameterized analysis for DC solution through the moment subspace.

The moments which are used for the DC analysis are not applicable for the parameterized analysis. For this analysis, the parameter is twisted with variables. Thus the new format is needed to be expanded in order to detect whether it is easy to compute the new moments. Using the proposed format, when $\alpha=0$ :

$$
\mathbf{G}(0) \mathbf{M}_{0}+\mathbf{f}\left(\mathbf{M}_{0}\right)=\mathbf{b}
$$

where $\mathbf{G}(0)$ is the $\mathbf{G}$ of original value with the corresponding resistor. From this equation, the solution of the zero-th moments equals to the result of the DC analysis. Thus the DC solution result could be used as the zero-th moment to find high order moments. Similar to the method in the previous chapter, firstly, taking the first order derivative of the nonlinear equation at the point $\alpha=0$ :

$$
\begin{aligned}
& \mathbf{G}(\alpha) \frac{d \mathbf{x}}{d \alpha}+\mathbf{G}^{\prime}(\alpha) \mathbf{x}+\frac{d \mathbf{f}(\mathbf{x})}{d \mathbf{x}} \frac{d \mathbf{x}}{d \alpha}=0 \\
& \mathbf{G}(\alpha) \frac{d \mathbf{x}}{d \alpha}+\mathbf{G}^{\prime} \mathbf{x}+\mathbf{J}(\mathbf{x}) \frac{d \mathbf{x}}{d \alpha}=0
\end{aligned}
$$

The $\mathbf{G}^{\prime}$ is a constant matrix, if the proposed formula is applied, the change of the circuit element is defined as the first order function of the $\alpha$. From the proposed formula, the parameter of $\alpha$ can be eliminated in the $\mathbf{G}^{\prime}$ matrix, and the item which has this matrix will not be a form of multiplication of two functions of $\alpha$. The 
Jacobian matrix could also be expanded as:

$$
\mathbf{J}(\mathbf{x}) \Rightarrow \mathbf{J}\left(\sum_{j=1}^{\infty} \mathbf{J}_{j} \alpha^{j}\right)
$$

where $\mathbf{J}_{j}$ is the $j$ th moment of the Jacobian matrix in respect of $\alpha$. Then the corresponding Taylor Series expansion is shown as:

$$
\begin{aligned}
& \mathbf{x}=\sum_{i=0}^{\infty} \mathbf{M}_{i} \alpha^{i} \\
& \frac{d \mathbf{x}}{d \alpha}=\sum_{i=1}^{\infty} i \mathbf{M}_{i} \alpha^{i-1} \\
& \mathbf{J}(\alpha)=\sum_{j=0}^{\infty} \mathbf{J}_{j} \alpha^{j}
\end{aligned}
$$

If these three Taylor Series expansion are used for the substitution of (7.6), the fundamental form for finding the high order moments is:

$$
\underbrace{\mathbf{G}(\alpha) \sum_{i=1}^{\infty} i \mathbf{M}_{i} \alpha^{i-1}}_{\text {first term }}+\underbrace{\mathbf{G}^{\prime} \sum_{i=0}^{\infty} \mathbf{M}_{i} \alpha^{i}}_{\text {second term }}+\underbrace{\sum_{i=1}^{\infty} \sum_{j=0}^{\infty} i \mathbf{J}_{j} \mathbf{M}_{i} \alpha^{i+j-1}}_{\text {third term }}=0
$$

The first order moment is:

$$
\left(\mathbf{G}(0)+\mathbf{J}_{0}\right) \mathbf{M}_{1}=-\mathbf{G}^{\prime} \mathbf{M}_{0}
$$

If (7.9) is taken as a high order derivative, higher order moments could be found. Note that the first term of the equation and the third term of the equation are multiplications of two functions of $\alpha$. The $\mathbf{G}^{\prime}$ for the second function is a constant matrix. 


\section{- High Order Derivative of the First Term}

If the (m-1)th order derivative is taken for the first term, the Leibniz Theorem does

not need to be applied fully because $\mathbf{G}(\alpha)$ is only the first order function of $\alpha$. Any

derivative which has an order bigger than 1 will equal to zero. Thus only two terms for this Leibniz Theorem expansion will be left:

$$
\begin{aligned}
\frac{d^{m-1}\left(\mathbf{G}(\alpha) \sum_{i=1}^{\infty} i \mathbf{M}_{i} \alpha^{i-1}\right)}{d \alpha^{m-1}} & =\sum_{r=0}^{m-1} \frac{(m-1) !}{r !(m-r-1) !} \frac{d^{r} \mathbf{G}(\alpha)}{d \alpha^{r}} \frac{d^{m-r-1} \sum_{i=1}^{\infty} i \mathbf{M}_{i} \alpha^{i-1}}{d \alpha^{m-r-1}} \\
& =\mathbf{G}(\alpha) m ! \mathbf{M}_{m}+(m-1) \mathbf{G}^{\prime}(m-1) ! \mathbf{M}_{m-1}
\end{aligned}
$$

\section{- High Order Derivative of the Second Term}

The (m-1)th order derivative of the second term of (7.9) is:

$$
\frac{d^{m-1}\left(\mathbf{G}^{\prime} \sum_{i=1}^{\infty} \mathbf{M}_{i} \alpha^{i}\right)}{d \alpha^{m-1}}=\mathbf{G}^{\prime}(m-1) ! \mathbf{M}_{m-1}
$$

\section{- High Order Derivative of the Third Term}

The (m-1)th order derivative of the third term of (7.9) has the same result with the Leibniz Theorem expansion as discussed in the previous chapter. The result will be:

$$
\frac{d^{m-1}\left(\sum_{i=1}^{\infty} \sum_{j=0}^{\infty} i \mathbf{J}_{j} \mathbf{M}_{i} \alpha^{i+j-1}\right)}{d \alpha^{m-1}}=n ! \mathbf{J}_{0} \mathbf{M}_{m}+\sum_{r=0}^{m-2}(m-1) !(r+1) \mathbf{J}_{m-r-1} \mathbf{M}_{r+1}
$$




\section{- High Order Moment Computational Format}

If the three results above are placed together, the format to find the high order moments is:

$$
\begin{aligned}
\mathbf{G}(\alpha) m ! \mathbf{M}_{m}+ & (m-1) \mathbf{G}^{\prime}(m-1) ! \mathbf{M}_{m-1}+\mathbf{G}^{\prime}(m-1) ! \mathbf{M}_{m-1}+n ! \mathbf{J}_{0} \mathbf{M}_{m} \\
& +\sum_{r=0}^{m-2}(m-1) !(r+1) \mathbf{J}_{m-r-1} \mathbf{M}_{r+1}=0
\end{aligned}
$$

The final result is:

$$
\left(\mathbf{G}(0)+\mathbf{J}_{0}\right) \mathbf{M}_{m}=-\left(\mathbf{G}^{\prime} \mathbf{M}_{m-1}+\frac{1}{m} \sum_{r=0}^{m-2}(r+1) \mathbf{J}_{m-r-1} \mathbf{M}_{r+1}\right), \quad m>1
$$

Note that in order to find the high order moments, only the inverse of $\left(\mathbf{G}(0)+\mathbf{J}_{0}\right)$ is required, so only one LU decomposition is needed. There is another point that the source vector $\mathbf{b}$ disappears in the process to find the first order moment, and the first order format is the special case required to find the high order moments.

To solve the nonlinear equation, methods to find the nonlinear moments and the Jacobian moments is the same as that which was illustrated in the previous chapter. However, the first nonlinear moment and the Jacobian moment should use the variable value of the DC solution. For the simulation point of view, the method to use the moment subspace DEIM will be same with the DC solution, but it is required that $\mathbf{G}(\alpha)$ is reduced for every step. The simulation result and further illustration will be provided in the next section. 


\subsection{Computation Results and Verification}

In this section, the previously described two examples will be used to verify the proposed method to conduct the parameterized analysis for the DC solution.

\subsubsection{Example I}

The changing of the parameter has two directions, positive and negative, and the result will be shown respectively. For these two directions, they will use same moments and nonlinear moments because $\alpha$ is expanded at 0 . Results could have a good estimation for the area closes to the expansion point.

For the BJT amplifier circuit, in which the parameter is changed to the positive side, the result is shown in figure 7.1. The result for the negative side is shown in figure 7.3. For the positive part, $g_{2}$ will be increased to twice its original value when $\alpha=1$. For the negative part, the parameter could not reach the end until -1 . If it reaches the -1 , it means $R_{2}$ will be infinity and it causes the ill conditioning. The maximum range is until $\alpha=-0.8$. The simulation result is: 


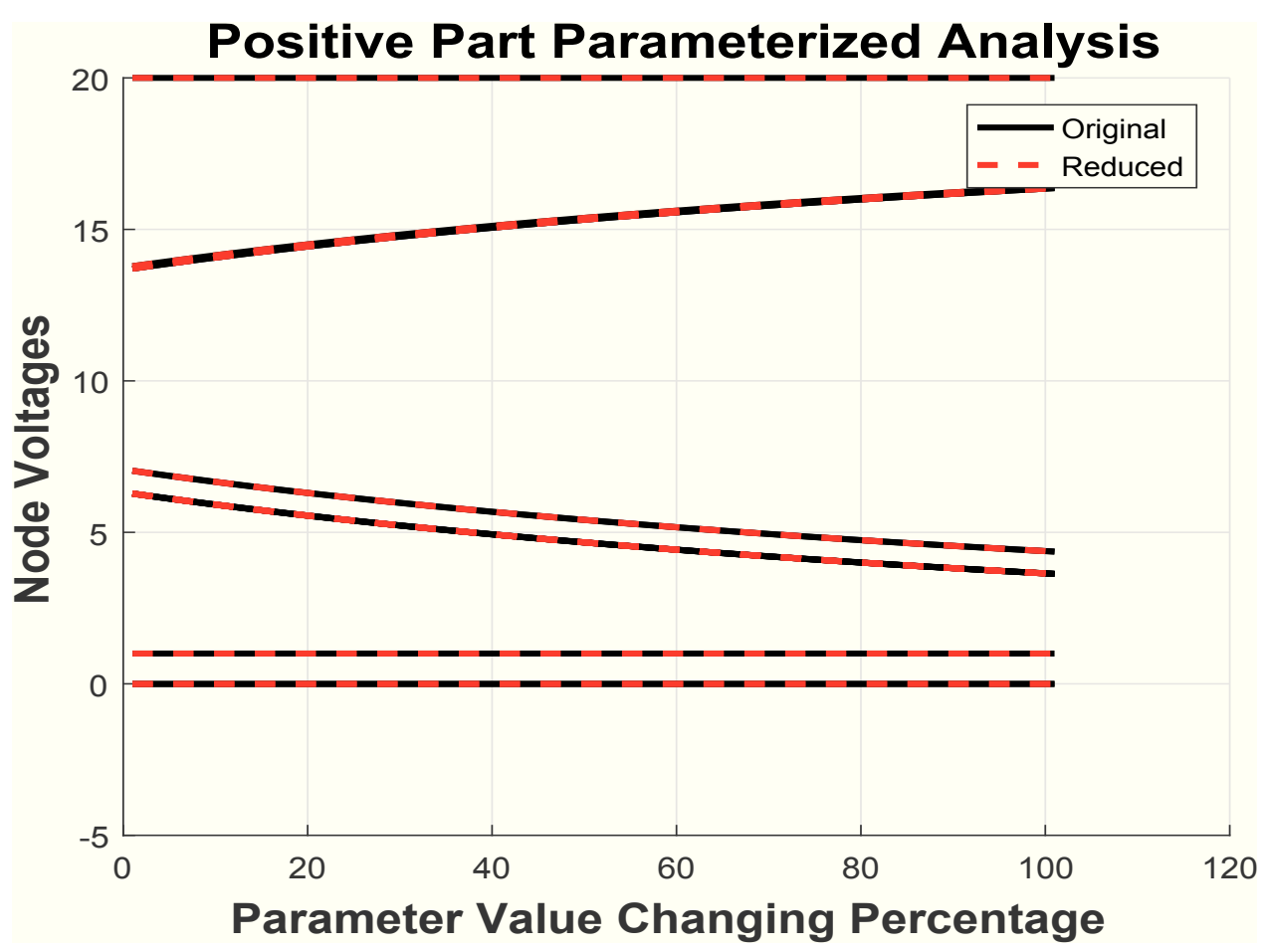

Figure 7.1: Example I: Positive Parameterized Analysis Result

The number of total iteration, speed and the moment number used is shown in table 7.1. The total moments of the reduced model are 3 , and it only needs to select 2 entries out of 19. The speed up is not dramatically increased because for every step Newton-Raphson iteration, the $\mathbf{G}$ matrix needs to to be reduced. The time saved from the moment subspace DEIM is insignificant compared with the MOR computation. The results show that the moment could be used to perform the parameterized analysis.

Table 7.1: Example I: Positive Parameterized Analysis

\begin{tabular}{|c|c|c|c|}
\hline & Model Size & Iteration Number & CPU time (s) \\
\hline Original Method & 19 & 52 & 0.0647 \\
\hline $\begin{array}{c}\text { Moment Subspace } \\
\text { DEIM Method }\end{array}$ & Moments: 3 & 50 & $\begin{array}{c}\text { Build ROM: } 0.0133 \\
\text { Nimulation: } 0.0303\end{array}$ \\
\hline
\end{tabular}

The value of the variable from the original method and the proposed method could 
be summarized, it is based on the changing of $g_{2}$.

\begin{tabular}{|c|c|c|c|c|c|c|c|c|c|}
\hline \multicolumn{9}{|c|}{ Positive Direction Parameter Changing (3 Moments, 2 Selections) } \\
\hline $\begin{array}{c}\text { Variable } \\
\text { Number }\end{array}$ & \multicolumn{3}{|c|}{$30 \%$} & \multicolumn{3}{c|}{$50 \%$} & \multicolumn{3}{c|}{$100 \%$} \\
\hline & Original & Proposed & Error & Original & Proposed & Error & Original & Proposed & Error \\
\hline 1 & 20 & 19.99996 & $4 \mathrm{E}-05$ & 20 & 19.99991 & $9 \mathrm{E}-05$ & 20 & 19.99972 & 0.00028 \\
\hline 2 & 1 & 1 & 0 & 1 & 1 & 0 & 1 & 0.99999 & $1 \mathrm{E}-05$ \\
\hline 3 & 5.94605 & 5.94593 & 0.00012 & 5.38914 & 5.38887 & 0.00027 & 4.36664 & 4.36598 & 0.00066 \\
\hline 4 & 14.85863 & 14.85878 & 0.00015 & 15.40573 & 15.40607 & 0.00034 & 16.40923 & 16.41009 & 0.00086 \\
\hline 5 & 5.1933 & 5.19311 & 0.00019 & 4.64067 & 4.64024 & 0.00043 & 3.62704 & 3.62589 & 0.00115 \\
\hline 6 & 0 & 0 & 0 & 0 & 0 & 0 & 0 & 0 & 0 \\
\hline 7 & 5.93913 & 5.93894 & 0.00019 & 5.38295 & 5.38252 & 0.00043 & 4.36181 & 4.36065 & 0.00116 \\
\hline 8 & 14.79008 & 14.79023 & 0.00015 & 15.34448 & 15.34482 & 0.00034 & 16.36135 & 16.36223 & 0.00088 \\
\hline 9 & 5.20023 & 5.20004 & 0.00019 & 4.64686 & 4.64643 & 0.00043 & 3.63188 & 3.63072 & 0.00116 \\
\hline 10 & 14.79008 & 14.79023 & 0.00015 & 15.34448 & 15.34482 & 0.00034 & 16.36135 & 16.36223 & 0.00088 \\
\hline 11 & 5.20023 & 5.20004 & 0.00019 & 4.64686 & 4.64643 & 0.00043 & 3.63188 & 3.63072 & 0.00116 \\
\hline 12 & 5.1933 & 5.19311 & 0.00019 & 4.64067 & 4.64024 & 0.00043 & 3.62704 & 3.62589 & 0.00115 \\
\hline 13 & 5.1933 & 5.19311 & 0.00019 & 4.64067 & 4.64024 & 0.00043 & 3.62704 & 3.62589 & 0.00115 \\
\hline 14 & -0.00857 & -0.00857 & 0 & -0.00791 & -0.00791 & 0 & -0.00669 & -0.00669 & 0 \\
\hline 15 & 0 & 0 & 0 & 0 & 0 & 0 & 0 & 0 & 0 \\
\hline 16 & 0.00692 & 0.00692 & 0 & 0.00619 & 0.00619 & 0 & 0.00484 & 0.00483 & $1 \mathrm{E}-05$ \\
\hline 17 & 0 & 0 & 0 & 0 & 0 & 0 & 0 & 0 & 0 \\
\hline 18 & 0.00692 & 0.00692 & 0 & 0.00619 & 0.00619 & 0 & 0.00484 & 0.00483 & $1 \mathrm{E}-05$ \\
\hline 19 & 0 & 0 & 0 & 0 & 0 & 0 & 0 & 0 & 0 \\
\hline
\end{tabular}

Figure 7.2: Example I: Positive Parameterized Analysis Result

For the negative direction: 


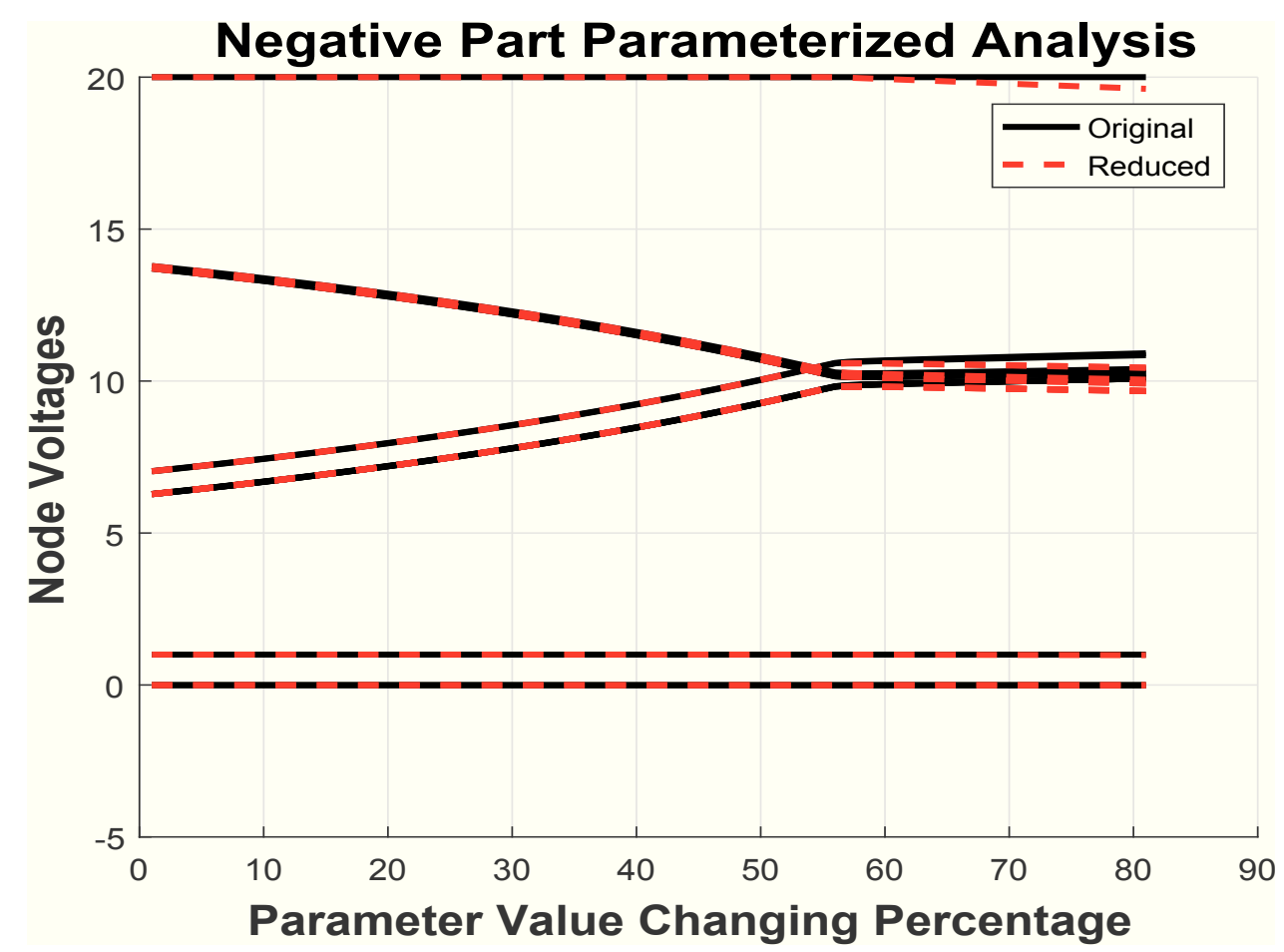

Figure 7.3: Example I: Negative Parameterized Analysis Result

The speed, iterations and the moment number requirement for the negative part is:

Table 7.2: Example I: Negative Parameterized Analysis

\begin{tabular}{|c|c|c|c|}
\hline & Model Size & Iteration Number & CPU time (s) \\
\hline Original Method & 19 & 91 & 0.0224 \\
\hline $\begin{array}{c}\text { Moment Subspace } \\
\text { DEIM Method }\end{array}$ & Moments: 3 & 81 & Build ROM: 0.0133 \\
& Nimulation: 0.0177 \\
\hline
\end{tabular}

The value of the variable is summarized as: 


\begin{tabular}{|c|c|c|c|c|c|c|c|c|c|}
\hline \multicolumn{9}{|c|}{ Negative Direction Parameter Changing (3 Moments, 2 Selections) } \\
\hline Variable & \multicolumn{3}{|c|}{$30 \%$} & \multicolumn{3}{c|}{$50 \%$} & \multicolumn{3}{c|}{$80 \%$} \\
\cline { 2 - 10 } Number & Original & Proposed & Error & Original & Proposed & Error & Original & Proposed & Error \\
\hline 1 & 20 & 19.99994 & $6 \mathrm{E}-05$ & 20 & 19.9998 & 0.0002 & 20 & 19.61664 & 0.38336 \\
\hline 2 & 1 & 1 & 0 & 1 & 0.99999 & $1 \mathrm{E}-05$ & 1 & 0.98083 & 0.01917 \\
\hline 3 & 8.61757 & 8.61729 & 0.00028 & 10.13543 & 10.13431 & 0.00112 & 10.91447 & 10.44533 & 0.46914 \\
\hline 4 & 12.23105 & 12.23137 & 0.00032 & 10.73671 & 10.73796 & 0.00125 & 10.40149 & 10.04716 & 0.35433 \\
\hline 5 & 7.84743 & 7.84704 & 0.00039 & 9.35686 & 9.35539 & 0.00147 & 10.08117 & 9.66614 & 0.41503 \\
\hline 6 & 0 & 0 & 0 & 0 & 0 & 0 & 0 & 0 & 0 \\
\hline 7 & 8.60711 & 8.60673 & 0.00038 & 10.12296 & 10.12148 & 0.00148 & 10.85011 & 10.43433 & 0.41578 \\
\hline 8 & 12.12746 & 12.12779 & 0.00033 & 10.61319 & 10.61447 & 0.00128 & 10.27351 & 9.91957 & 0.35394 \\
\hline 9 & 7.85789 & 7.8575 & 0.00039 & 9.36934 & 9.36786 & 0.00148 & 10.09461 & 9.67903 & 0.41558 \\
\hline 10 & 12.12746 & 12.12779 & 0.00033 & 10.61319 & 10.61447 & 0.00128 & 10.27351 & 9.91957 & 0.35394 \\
\hline 11 & 7.85789 & 7.8575 & 0.00039 & 9.36934 & 9.36786 & 0.00148 & 10.09461 & 9.67903 & 0.41558 \\
\hline 12 & 7.84743 & 7.84704 & 0.00039 & 9.35686 & 9.35539 & 0.00147 & 10.08117 & 9.66614 & 0.41503 \\
\hline 13 & 7.84743 & 7.84704 & 0.00039 & 9.35686 & 9.35539 & 0.00147 & 10.08117 & 9.66614 & 0.41503 \\
\hline 14 & -0.01175 & -0.01175 & 0 & -0.01355 & -0.01355 & 0 & -0.01391 & -0.01388 & $3 \mathrm{E}-05$ \\
\hline 15 & 0 & 0 & 0 & 0 & 0 & 0 & 0 & 0 & 0 \\
\hline 16 & 0.01046 & 0.01046 & 0 & 0.01248 & 0.01247 & $1 \mathrm{E}-05$ & 0.01344 & 0.01289 & 0.00055 \\
\hline 17 & 0 & 0 & 0 & 0 & 0 & 0 & 0.00052 & 0 & 0.00052 \\
\hline 18 & 0.01046 & 0.01046 & 0 & 0.01248 & 0.01247 & $1 \mathrm{E}-05$ & 0.01345 & 0.01289 & 0.00056 \\
\hline 19 & 0 & 0 & 0 & 0 & 0 & 0 & 0 & 0 & 0 \\
\hline
\end{tabular}

Figure 7.4: Example I: Negative Parameterized Analysis Result

From the result which is shown above, the maximum step size for the positive parameterized analysis can reach to $\alpha=1$. The maximum step size for the negative direction is $\alpha=-0.6$. Any value of this circuit element within this range can also be solved by using the proposed method by only one step of Newton-Raphson iteration. Both the original method and the proposed method need the same iteration number to solve the circuit.

\subsubsection{Example II}

For the 201 nodes diode ladder circuit, the same steps will be taken as described in Example I. The parameter relates with the conductance of an element connected with 25 th node. Figure 7.5 shows the positive side parameterized result. It picks 12 
variables out of 201 for the plotting.

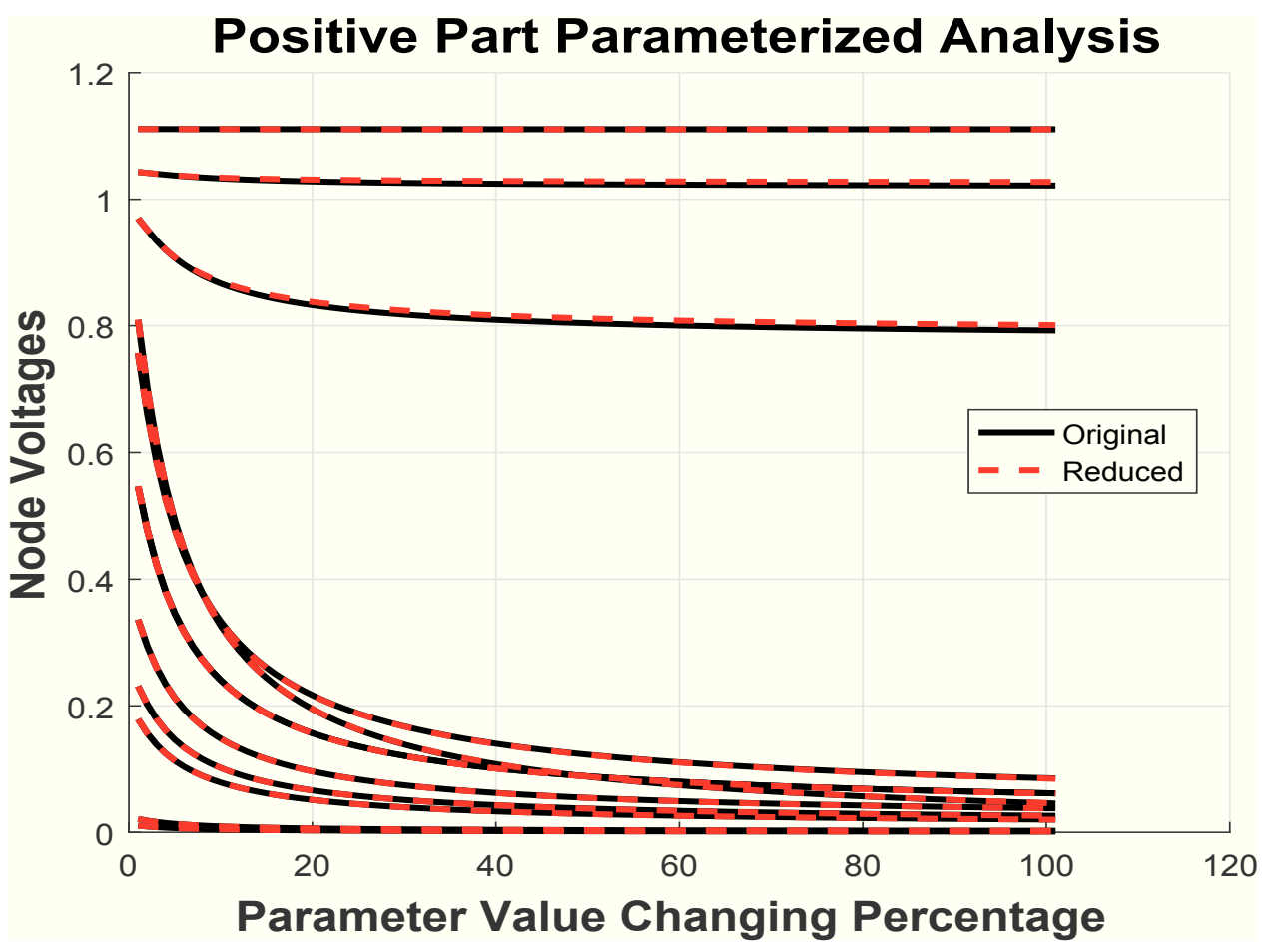

Figure 7.5: Example II: Positive Parameterized Analysis

The speed, total iteration number and the moments requirement for the positive direction are:

Table 7.3: Example II: Positive Parameterized Analysis

\begin{tabular}{|c|c|c|c|}
\hline & Model Size & Iteration Number & CPU time (s) \\
\hline Original Method & 201 & 467 & 0.6468 \\
\hline $\begin{array}{c}\text { Moment Subspace } \\
\text { DEIM Method }\end{array}$ & Nonlinear Selection: 5 & 440 & $\begin{array}{c}\text { Build ROM: 0.0262 } \\
\text { Simulation: 0.1571 }\end{array}$ \\
\hline
\end{tabular}

The value of the variable from the original method and the proposed method could be summarized: 


\begin{tabular}{|c|c|c|c|c|c|c|c|c|c|}
\hline \multicolumn{9}{|c|}{ Positive Direction Parameter Changing (5 moments, 5 selections) } \\
\hline \multirow{2}{*}{$\begin{array}{c}\text { Variable } \\
\text { Number }\end{array}$} & \multicolumn{3}{|c|}{$30 \%$} & \multicolumn{3}{c|}{$50 \%$} & \multicolumn{3}{c|}{$100 \%$} \\
\hline & Original & Proposed & Error & Original & Proposed & Error & Original & Proposed & Error \\
\hline 2 & 1.1107 & 1.11051 & 0.00019 & 1.11068 & 1.11045 & 0.00023 & 1.11066 & 1.11039 & 0.00027 \\
\hline 5 & 1.02576 & 1.0286 & 0.00284 & 1.02367 & 1.02724 & 0.00357 & 1.02181 & 1.02607 & 0.00426 \\
\hline 10 & 0.81655 & 0.81641 & 0.00014 & 0.80346 & 0.80346 & 0 & 0.79231 & 0.79244 & 0.00013 \\
\hline 25 & 0.13489 & 0.13519 & 0.0003 & 0.08647 & 0.08671 & 0.00024 & 0.04556 & 0.04571 & 0.00015 \\
\hline 50 & 0.11843 & 0.11735 & 0.00108 & 0.08759 & 0.0863 & 0.00129 & 0.06154 & 0.06003 & 0.00151 \\
\hline 70 & 0.07288 & 0.07222 & 0.00066 & 0.0539 & 0.05311 & 0.00079 & 0.03787 & 0.03694 & 0.00093 \\
\hline 85 & 0.03872 & 0.03837 & 0.00035 & 0.02864 & 0.02821 & 0.00043 & 0.02012 & 0.01963 & 0.00049 \\
\hline 100 & 0.00455 & 0.00451 & $4 \mathrm{E}-05$ & 0.00337 & 0.00332 & $5 \mathrm{E}-05$ & 0.00237 & 0.00231 & $6 \mathrm{E}-05$ \\
\hline 130 & 0.16398 & 0.16258 & 0.0014 & 0.12128 & 0.11959 & 0.00169 & 0.08521 & 0.08324 & 0.00197 \\
\hline 150 & 0.11843 & 0.11735 & 0.00108 & 0.08759 & 0.0863 & 0.00129 & 0.06154 & 0.06003 & 0.00151 \\
\hline 180 & 0.0501 & 0.04965 & 0.00045 & 0.03706 & 0.03651 & 0.00055 & 0.02604 & 0.0254 & 0.00064 \\
\hline 201 & 0.00228 & 0.00226 & $2 \mathrm{E}-05$ & 0.00168 & 0.00166 & $2 \mathrm{E}-05$ & 0.00118 & 0.00115 & $3 \mathrm{E}-05$ \\
\hline
\end{tabular}

Figure 7.6: Example II: Positive Parameterized Analysis Result

And if the parameter is reduced, the results are shown in figure 7.10.

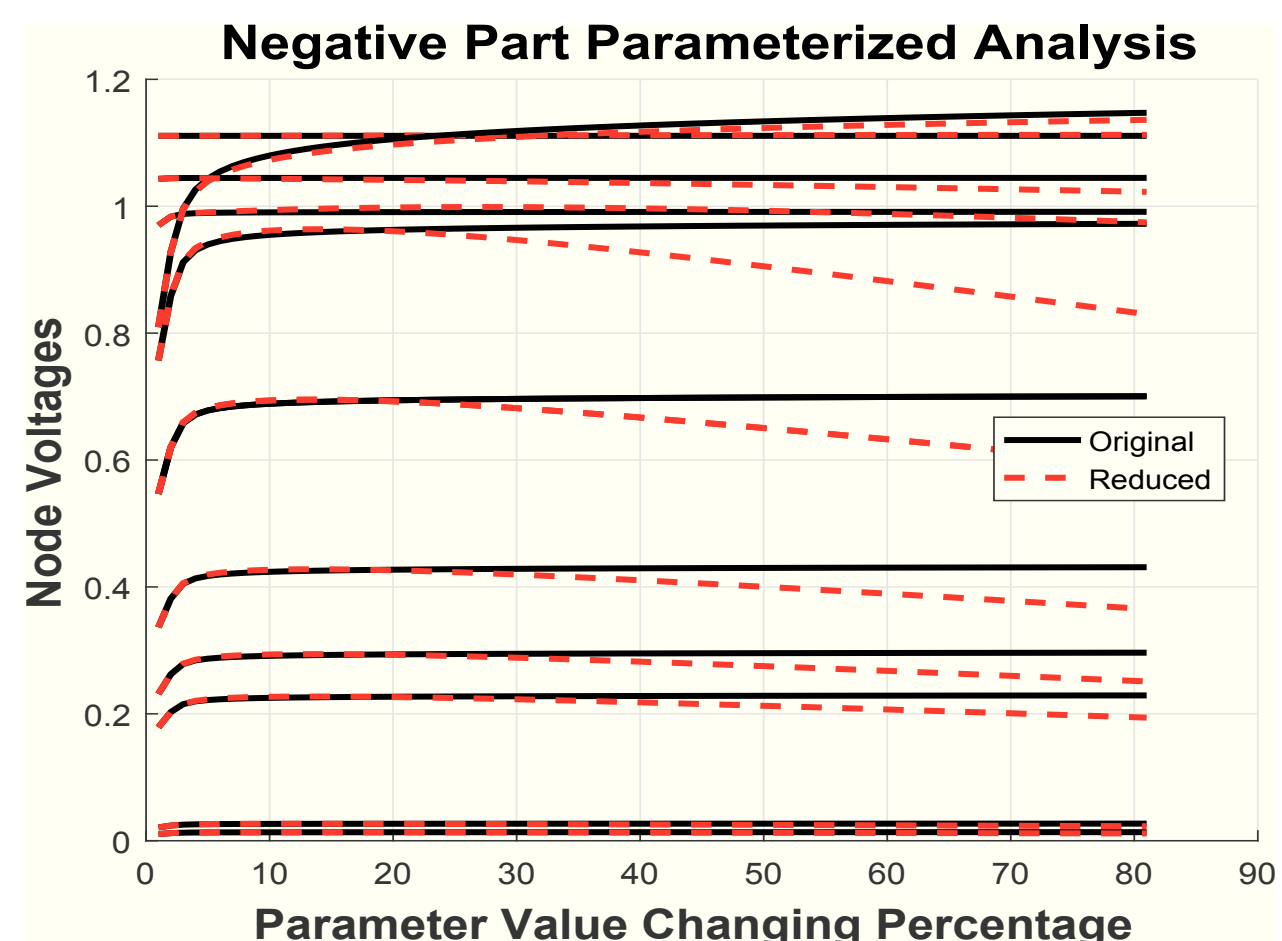

Figure 7.7: Example II: Negative Parameterized Analysis with 5 Moments-10 Selection 
The speed and iterations are measured for 5 moments and 10 nonlinear selections.

Table 7.4: Example II: Negative Parameterized Analysis

\begin{tabular}{|c|c|c|c|}
\hline & Model Size & Iteration Number & CPU time (s) \\
\hline Original Method & 201 & 272 & 0.3742 \\
\hline $\begin{array}{c}\text { Moment Subspace } \\
\text { DEIM Method }\end{array}$ & Moments: 5 & 231 & $\begin{array}{c}\text { Build ROM: } 0.0380 \\
\text { Simulation: } 0.0821\end{array}$ \\
\hline
\end{tabular}

The speed up factor is not dramatically increased compared with the DC solution. This is due to the need for the $\mathbf{G}$ matrix to ramp and it also needs to be reduced for each step. The value of the variable is:

\begin{tabular}{|c|c|c|c|c|c|c|c|c|c|}
\hline & \multicolumn{6}{|c|}{ Negative Direction Parameter Changing (5 moments, 10 selections) } \\
\hline Variable & \multicolumn{3}{|c|}{$10 \%$} & \multicolumn{3}{c|}{$20 \%$} & \multicolumn{4}{c|}{$40 \%$} \\
\cline { 2 - 10 } Number & Original & Proposed & Error & Original & Proposed & Error & Original & Proposed & Error \\
\hline 2 & 1.11096 & 1.1112 & 0.00024 & 1.11096 & 1.11142 & 0.00046 & 1.11096 & 1.11188 & 0.00092 \\
\hline 5 & 1.04461 & 1.04309 & 0.00152 & 1.04463 & 1.04132 & 0.00331 & 1.04465 & 1.03627 & 0.00838 \\
\hline 10 & 0.99039 & 0.99445 & 0.00406 & 0.99074 & 0.99821 & 0.00747 & 0.99099 & 0.99631 & 0.00532 \\
\hline 25 & 1.08385 & 1.07675 & 0.0071 & 1.10727 & 1.09861 & 0.00866 & 1.12797 & 1.11788 & 0.01009 \\
\hline 50 & 0.68961 & 0.69451 & 0.0049 & 0.69438 & 0.69173 & 0.00265 & 0.6978 & 0.66541 & 0.03239 \\
\hline 70 & 0.42437 & 0.42739 & 0.00302 & 0.42731 & 0.42568 & 0.00163 & 0.42941 & 0.40948 & 0.01993 \\
\hline 85 & 0.22545 & 0.22705 & 0.0016 & 0.22701 & 0.22614 & 0.00087 & 0.22813 & 0.21754 & 0.01059 \\
\hline 100 & 0.02652 & 0.02671 & 0.00019 & 0.02671 & 0.0266 & 0.00011 & 0.02684 & 0.02559 & 0.00125 \\
\hline 130 & 0.95619 & 0.9626 & 0.00641 & 0.96319 & 0.95963 & 0.00356 & 0.96827 & 0.92522 & 0.04305 \\
\hline 150 & 0.68961 & 0.69451 & 0.0049 & 0.69438 & 0.69173 & 0.00265 & 0.6978 & 0.66541 & 0.03239 \\
\hline 180 & 0.29176 & 0.29383 & 0.00207 & 0.29378 & 0.29265 & 0.00113 & 0.29522 & 0.28152 & 0.0137 \\
\hline 201 & 0.01326 & 0.01336 & 0.0001 & 0.01335 & 0.0133 & $5 \mathrm{E}-05$ & 0.01342 & 0.0128 & 0.00062 \\
\hline
\end{tabular}

Figure 7.8: Example II: Negative Parameterized Analysis Result with 5 Moments10 Selection

It could give the approximated result until $\alpha=-0.4$, but if more moments and nonlinear moments are used, the result would be: 


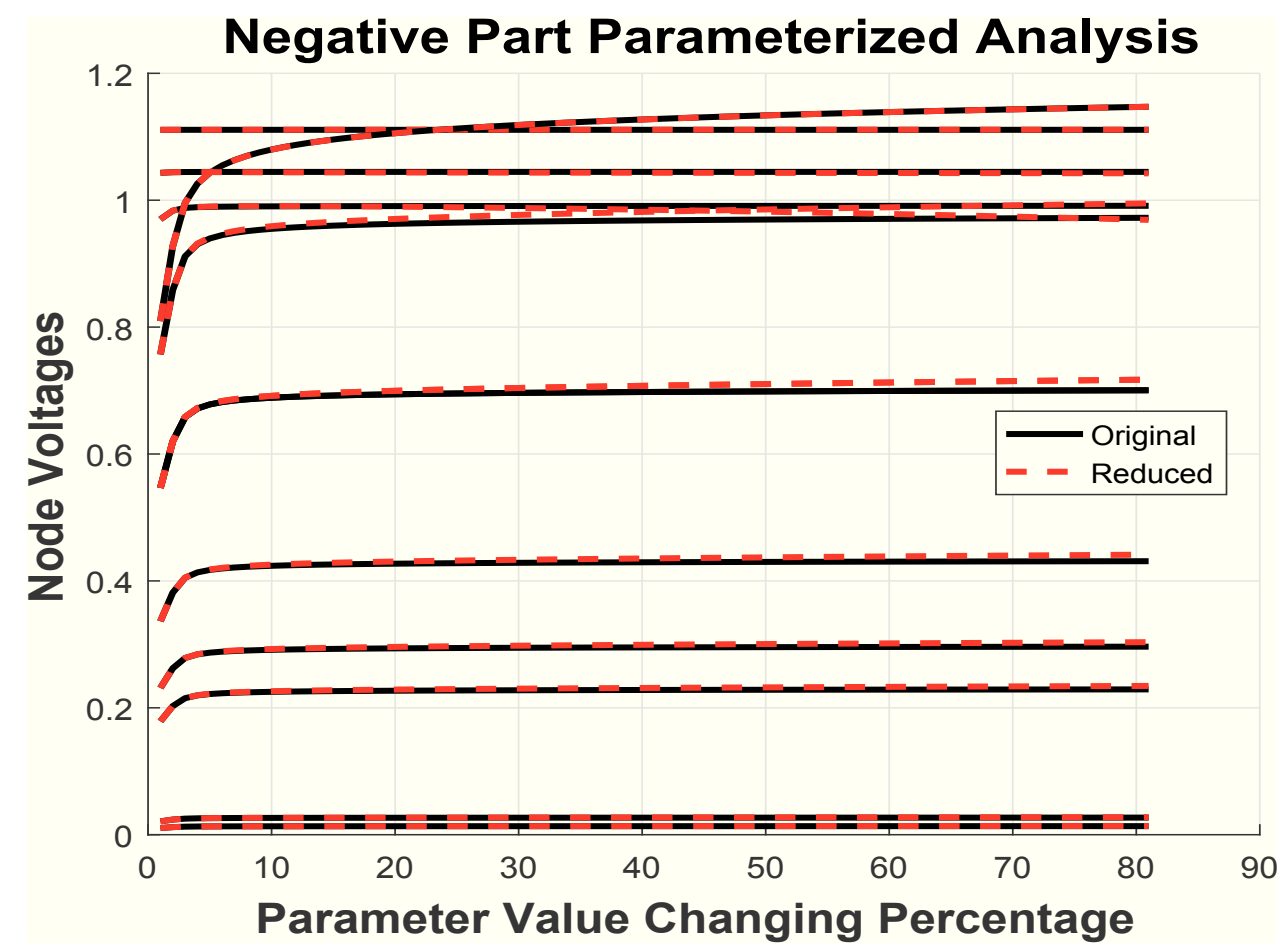

Figure 7.9: Example II: Negative Parameterized Analysis with 7 Moments-15 Selection

The value of the variable for 7 moments and 15 selections negative part parameterized analysis is: 


\begin{tabular}{|c|c|c|c|c|c|c|c|c|c|}
\hline \multicolumn{9}{|c|}{ Negative Direction Parameter Changing (7 moments, 15 selections) } \\
\hline Variable & \multicolumn{3}{|c|}{$30 \%$} & \multicolumn{3}{c|}{$50 \%$} & \multicolumn{3}{c|}{$80 \%$} \\
\cline { 2 - 10 } Number & Original & Proposed & Error & Original & Proposed & Error & Original & Proposed & Error \\
\hline 2 & 1.11096 & 1.1111 & 0.00014 & 1.11096 & 1.11113 & 0.00017 & 1.11096 & 1.11115 & 0.00019 \\
\hline 5 & 1.04464 & 1.04346 & 0.00118 & 1.04465 & 1.04308 & 0.00157 & 1.04466 & 1.04235 & 0.00231 \\
\hline 10 & 0.99089 & 0.98751 & 0.00338 & 0.99106 & 0.98158 & 0.00948 & 0.99119 & 0.96901 & 0.02218 \\
\hline 25 & 1.1196 & 1.11954 & $6 \mathrm{E}-05$ & 1.1343 & 1.13424 & $6 \mathrm{E}-05$ & 1.14729 & 1.1472 & $9 \mathrm{E}-05$ \\
\hline 50 & 0.69649 & 0.70443 & 0.00794 & 0.69872 & 0.71049 & 0.01177 & 0.70047 & 0.71719 & 0.01672 \\
\hline 70 & 0.42861 & 0.4335 & 0.00489 & 0.42998 & 0.43723 & 0.00725 & 0.43106 & 0.44134 & 0.01028 \\
\hline 85 & 0.2277 & 0.23029 & 0.00259 & 0.22843 & 0.23228 & 0.00385 & 0.229 & 0.23446 & 0.00546 \\
\hline 100 & 0.02679 & 0.02709 & 0.0003 & 0.02687 & 0.02733 & 0.00046 & 0.02694 & 0.02758 & 0.00064 \\
\hline 130 & 0.96633 & 0.97733 & 0.011 & 0.96966 & 0.98581 & 0.01615 & 0.97229 & 0.99501 & 0.02272 \\
\hline 150 & 0.69649 & 0.70443 & 0.00794 & 0.69872 & 0.71049 & 0.01177 & 0.70047 & 0.71719 & 0.01672 \\
\hline 180 & 0.29467 & 0.29803 & 0.00336 & 0.29561 & 0.30059 & 0.00498 & 0.29635 & 0.30342 & 0.00707 \\
\hline 201 & 0.01339 & 0.01355 & 0.00016 & 0.01344 & 0.01366 & 0.00022 & 0.01347 & 0.01379 & 0.00032 \\
\hline
\end{tabular}

Figure 7.10: Example II: Negative Parameterized Analysis Result with 7 Moments15 Selection

The result could have a much better approximation for smaller values of the parameter. However, as with example I, $\alpha$ could not reach 0 or it will cause the ill conditioning.

The biggest step size to do the negative part parameterized analysis is $4 \%$. This is not due to the moments quality. The DEIM theory also serves the parameterized analysis for the DC solution. Finally, the parameterized analysis could be used for all values of the source, and as soon as the corresponding nonlinear system response is known, the parameterized analysis for the current state of the circuit could be applied. 


\section{Chapter 8}

\section{Conclusion and Future Work}

\subsection{Conclusion}

The MOR for nonlinear circuits based on the projection technique is computationally expensive because of the following problems. The first problem is that it is difficult to find the relative moments based on a parameter. Both the determination of the parameter and the moment subspace are required to obtain the ROM. The second reason is that in order to solve the circuit, for every Newton-Raphson iteration, all nonlinear functions are needed to be built in the original domain.

In this thesis, an efficient method was presented to solve the DC analysis problem for nonlinear circuits. Additionally, the parameterized analysis method for the DC solution was illustrated. The key idea to solve the two issues above is to find the moment subspace for the variable, and to apply the DEIM theory to reduce the cost of MOR and the evaluation of nonlinear functions. The important issues for finding the moment subspace is not to find the moments of the nonlinear function, but the Jacobian matrix's high order derivatives will be obtained from the same order moments of the variable. Following that, an iteration loop, which only needs one LU decomposition can be used to locates the high order moments. The thesis verified that 
moments and nonlinear moments could be applied to use the DEIM theory for the MOR. Without circuit testing, the ROM could be found from the projection matrices and the approximated nonlinear function.

Additionally, the thesis presented a format of how to do the parameterized analysis. Using the similar techniques with the DC analysis, the corresponding moment subspace could also be found with a low computational cost. Numerical examples were presented to validate the proposed method for both the DC analysis and the parameterized analysis. The proposed method shows a relative speeding up comparing to the original source stepping method with high accuracy. 


\subsection{Future Work}

For future research, the following explorations are foreseen:

- The parameterized analysis could also have an opportunity to be applied for multiple parameters. The format could be obtained from the multiple parameter Taylor Series Expansion. This will give an opportunity to find the new steady response if the circuit elements change in different patterns.

- This method could be applied to a parameter variant nonlinear circuit, which has the characteristics that the parameter changes based on an exact function. It could be used for the analysis that the geometry of nonlinear model changes.

- The proposed method could have a chance to be applied for the transient analysis. It could be combined with the Harmonic Balance technique [34] the Piecewise Linear method solve the nonlinear circuit. Additionally, the method could be modified to find the moment subspace which will be used for the transient analysis. 


\section{List of References}

[1] A. Verhoeven, "Redundancy reduction of ic models by multirate time-integration and model order reduction," Dept. of Mathematics and Computer Science, University of Technology, Eindhoven, The Netherlands, 2008.

[2] L. T. Pillage, X. Huang, and R. A. Rohrer, "Awesim: Asymptotic waveform evaluation for timing analysis," in Design Automation, 1989. 26th Conference on, pp. 634-637, June 1989.

[3] S. Chaturantabut and D. C. Sorensen, "Nonlinear model reduction via discrete empirical interpolation," SIAM Journal on Scientific Computing, vol. 32, no. 5, pp. 2737-2764, 2010.

[4] M. Barrault, Y. Maday, N. C. Nguyen, and A. T. Patera, "An empirical interpolationmethod: application to efficient reduced-basis discretization of partial differential equations," Comptes Rendus Mathematique, vol. 339, no. 9, pp. 667$672,2004$.

[5] C.-W. Ho, A. Ruehli, and P. Brennan, "The modified nodal approach to network analysis," IEEE Transactions on Circuits and Systems, vol. 22, pp. 504-509, Jun 1975.

[6] K. Yamamura and S. Ishiguro, "Finding all dc solutions of nonlinear circuits using parallelogram lp test," in Circuit Theory and Design (ECCTD), 2015 European Conference on, pp. 1-4, Aug 2015.

[7] M. Tadeusiewicz and S. Halgas, "Analysis of bjt circuits having multiple dc solutions using deflation technique," in Signals and Electronic Systems (ICSES), 2012 International Conference on, pp. 1-4, Sept 2012.

[8] M. Tadeusiewicz and S. Halgas, "Finding all the dc solutions of circuits containing diodes and bipolar transistors," in Mixed Design of Integrated Circuits 
Systems, 2009. MIXDES '09. MIXDES-16th International Conference, pp. 433437, June 2009.

[9] R. C. Melville, L. Trajkovic, S. C. Fang, and L. T. Watson, "Artificial parameter homotopy methods for the dc operating point problem," IEEE Transactions on Computer-Aided Design of Integrated Circuits and Systems, vol. 12, pp. 861-877, Jun 1993.

[10] F. Najm, Circuit Simulation. Wiley, 2010.

[11] E. Gad and M. Nakhla, "Model reduction for dc solution of large nonlinear circuits," in Computer-Aided Design, 1999. Digest of Technical Papers. 1999 IEEE/ACM International Conference on, pp. 376-379, Nov 1999.

[12] H. A. v. d. V. Wilhelmus H. A. Schilders and J. Rommes, Model order reduction: theory, research aspects and applications., vol. 13. Springer Berlin Heidelberg, 2008.

[13] A. Antoulas, Approximation of Large-Scale Dynamical Systems. Society for Industrial and Applied Mathematics, 2005.

[14] Z. Bai, "Krylov subspace techniques for reduced-order modeling of large-scale dynamical systems," Appl. Numer. Math., vol. 43, pp. 9-44, Oct. 2002.

[15] R. W. Freund, "Passive reduced-order modeling via krylov-subspace methods," in Computer-Aided Control System Design, 2000. CACSD 2000. IEEE International Symposium on, pp. 261-266, 2000.

[16] A. Odabasioglu, M. Celik, and L. T. Pileggi, "Prima: passive reduced-order interconnect macromodeling algorithm," in Computer-Aided Design, 1997. Digest of Technical Papers., 1997 IEEE/ACM International Conference on, pp. 58-65, Nov 1997.

[17] A. Odabasioglu, M. Celik, and L. T. Pileggi, "Prima: passive reduced-order interconnect macromodeling algorithm," IEEE Transactions on Computer-Aided Design of Integrated Circuits and Systems, vol. 17, pp. 645-654, Aug 1998.

[18] C. W. Rowley, T. Colonius, and R. M. Murray, "Model reduction for compressible flows using $\{$ POD $\}$ and galerkin projection," Physica D: Nonlinear Phenomena, vol. 189, no. 12, pp. 115 - 129, 2004. 
[19] K. Kunisch and S. Volkwein, "Galerkin proper orthogonal decomposition methods for a general equation in fluid dynamics," SIAM Journal on Numerical analysis, vol. 40, no. 2, pp. 492-515, 2002.

[20] T. Bui-Thanh, M. Damodaran, and K. E. Willcox, "Aerodynamic data reconstruction and inverse design using proper orthogonal decomposition," AIAA journal, vol. 42, no. 8, pp. 1505-1516, 2004.

[21] K. Kunisch and S. Volkwein, "Control of the burgers equation by a reducedorder approach using proper orthogonal decomposition," Journal of Optimization Theory and Applications, vol. 102, no. 2, pp. 345-371, 1999.

[22] Y. Liu, W. Yuan, and H. Chang, "A global maximum error controller-based method for linearization point selection in trajectory piecewise-linear model order reduction," IEEE Transactions on Computer-Aided Design of Integrated Circuits and Systems, vol. 33, pp. 1100-1104, July 2014.

[23] D. Ropers, V. Baldazzi, and H. de Jong, "Model reduction using piecewise-linear approximations preserves dynamic properties of the carbon starvation response in escherichia coli," IEEE/ACM Transactions on Computational Biology and Bioinformatics, vol. 8, pp. 166-181, Jan 2011.

[24] B. N. Bond and L. Daniel, "A piecewise-linear moment-matching approach to parameterized model-order reduction for highly nonlinear systems," IEEE Transactions on Computer-Aided Design of Integrated Circuits and Systems, vol. 26, pp. 2116-2129, Dec 2007.

[25] H. Flanders, "Differentiation under the integral sign," The American Mathematical Monthly, vol. 80, no. 6, pp. 615-627, 1973.

[26] T. Henneron and S. Clnet, "Application of the pgd and deim to solve a 3-d non-linear magnetostatic problem coupled with the circuit equations," IEEE Transactions on Magnetics, vol. 52, pp. 1-4, March 2016.

[27] T. Henneron and S. Clnet, "Model-order reduction of multiple-input non-linear systems based on pod and dei methods," IEEE Transactions on Magnetics, vol. 51, pp. 1-4, March 2015.

[28] D. Amsallem and J. Nordstrom, "Energy stable model reduction of neurons by nonnegative discrete empirical interpolation," SIAM Journal on Scientific Computing, vol. 38, no. 2, pp. B297-B326, 2016. 
[29] A. Brambilla and G. Storti-Gajani, "Frequency warping in time-domain circuit simulation," IEEE Transactions on Circuits and Systems I: Fundamental Theory and Applications, vol. 50, no. 7, pp. 904-913, 2003.

[30] F. N. Najm, Circuit simulation. John Wiley \& Sons, 2010.

[31] R. Griffith and M. Nakhla, "A new high-order absolutely-stable explicit numerical integration algorithm for the time-domain simulation of nonlinear circuits," in Proc. IEEE/ACM Int. Conf. Comput.-Aided Design, (San Jose, CA, USA), pp. 276-280, Nov. 1997.

[32] H. Antil, M. Heinkenschloss, and D. C. Sorensen, "Application of the discrete empirical interpolation method to reduced order modeling of nonlinear and parametric systems," in Reduced order methods for modeling and computational reduction, pp. 101-136, Springer, 2014.

[33] F. Negri, A. Manzoni, and D. Amsallem, "Efficient model reduction of parametrized systems by matrix discrete empirical interpolation," Journal of Computational Physics, vol. 303, pp. 431-454, 2015.

[34] B. Bandali, E. Gad, and M. Bolic, "Accelerated harmonic-balance analysis using a graphical processing unit platform," IEEE Transactions on Computer-Aided Design of Integrated Circuits and Systems, vol. 33, pp. 1017-1030, July 2014. 